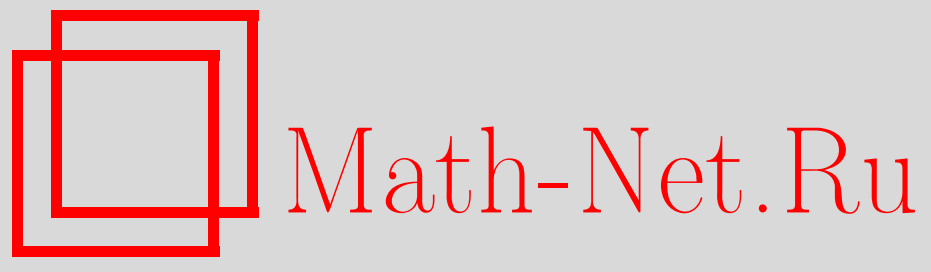

А. Р. Алимов, И. Г. Царьков, Связность и солнечность в задачах наилучшего и почти наилучшего приближения, УМН, 2016, том 71, выпуск 1, 3-84

DOI: https://doi.org/10.4213/rm9698

Использование Общероссийского математического портала Math-Net.Ru подразумевает, что вы прочитали и согласны с пользовательским соглашением http://www.mathnet.ru/rus/agreement

Параметры загрузки:

IP : 3.85 .73 .92

26 апреля 2023 г., 15:20:51

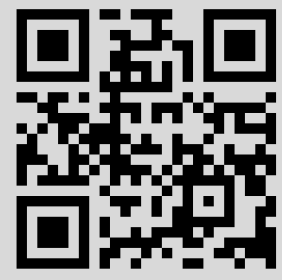




\title{
Связность и солнечность в задачах наилучшего и почти наилучшего приближения
}

\begin{abstract}
А. Р. Алимов, И. Г. Царьков
В обзоре рассматриваются структурные характеристики "солнц" в линейных нормированных пространствах. Особый упор делается на свойства связности и монотонной линейной связности солнц. Рассматриваются как прямые теоремы геометрической теории приближений, в которых из структурных характеристик множеств выводят их аппроксимативные свойства, так и обратные теоремы, в которых из аппроксимативных свойств множеств получают их структурные характеристики. Геометрические методы теории приближений используются для нахождения решений уравнения эйконала.

Библиография: 231 название.
\end{abstract}

Ключевые слова: солнце, строгое солнце, чебышёвское множество, почти наилучшее приближение, связность, бесконечная связность, монотонная линейная связность, уравнение эйконала.

DOI: $10.4213 / \mathrm{rm} 9698$

\section{СоДЕРЖАНИЕ}

1. Приближение коротких длин волн. Уравнение эйконала ........... 4

1.1. Структура особого множества на плоскости .............. 7

1.2. $C^{1}$-решения уравнения эйконала. Связь с солнечностью ...... 9

2. Обзор основных понятий и определений геометрической теории прибли-

жений ............................................. 12

3. Свойства отделимости для солнца. Глобальный минимизатор и луна . 20

3.1. Отделимость конусом для солнц ..................... 20

3.2. Солнца и критерий Колмогорова ближайшего элемента ......... 21

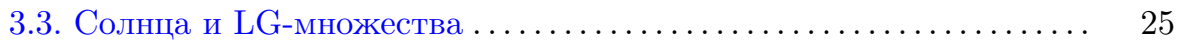

3.4. Характеризации солнц в различных пространствах .......... 26

4. Классы солнц, соотношения между ними и выпуклость. Точки свети-

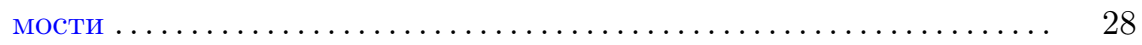

5. Классы связности. Связность чебышёвских множеств и множеств един-

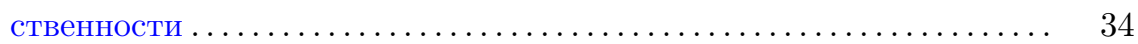

6. Понятия ацикличности и клеточноподобности множеств ........... 39

Работа выполнена при поддержке РФФИ (грант № 16-01-00295). 
7. Выборки из оператора наилучшего и почти наилучшего приближения.

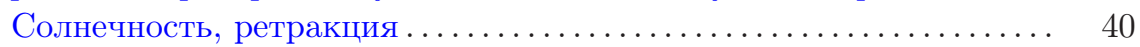

8. Классы солнц и связность ............................ 49

8.1. Связность в конечномерных пространствах............... 50

8.2. Связность в бесконечномерных пространствах ............ 51

8.3. Пример Кощеева несвязного солнца ................... 54

8.4. Связность пересечений солнц и чебышёвских множеств с подпространствами и промежутками в пространствах $C(Q) \ldots \ldots$

8.5. Количество компонент связности дополнения к чебышёвским

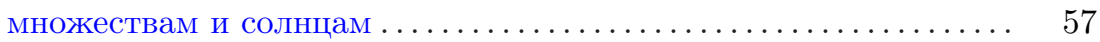

9. Монотонная линейная связность и т-связность ................ 59

9.1. Свойства монотонно связных и т-связных множеств ......... 59

9.2. Солнечность и монотонная линейная связность ............ 64

9.3. (ВМ)-пространства и их свойства............................ 65

9.4. Монотонная линейная связность и т-связность солнц в конечномерных (BM)-пространствах . .................. 67

9.5. Солнечность и монотонная линейная связность в пространствах $C(Q) \ldots \ldots \ldots \ldots \ldots \ldots \ldots \ldots \ldots \ldots \ldots \ldots \ldots \ldots \ldots \ldots \ldots \ldots \ldots \ldots \ldots \ldots \ldots \ldots \ldots \ldots \ldots$

9.6. Пересечение солнц и монотонно линейно связных множеств

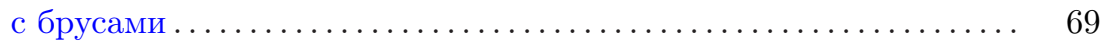

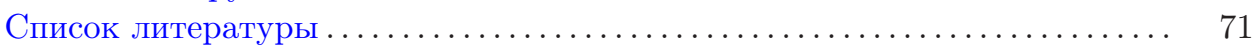

\section{1. Приближение коротких длин волн. Уравнение эйконала}

Одно из интересных направлений геометрической теории приближения, использующее понятие солнечности, связано с уравнениями Гамильтона-Якоби. Рассмотрим это на простейшем примере - уравнении эйконала, представляющем собой основное уравнение геометрической оптики:

$$
|\nabla f(x)|=n,
$$

где $n=n(x)$ - показатель преломления. Здесь и далее $|\cdot|$ - стандартная евклидова норма на $\mathbb{R}^{n}$. Напомним, что геометрическая оптика - это раздел оптики, в котором предполагается, что длина волны пренебрежимо мала, а уравнение эйконала получается из волнового уравнения для комплексной амплитуды (уравнения Гельмгольца). Функцию $f$ называют эйконалом ${ }^{1}$. Поверхности $f=$ const называются геометрическими волновыми поверхностями или геометрическими волновыми фронтами. Уравнение эйконала можно рассматривать также как уравнение Гамильтона-Якоби для вариационной задачи

$$
\delta \int n d s=0,
$$

впервые поставленной применительно к оптике Ферма (принципа наикратчайшего оптического пути).

\footnotetext{
${ }^{1}$ Термин "эйконал” (от греческого слова $\varepsilon i \varkappa \bar{\omega} \nu$ - изображение) был введен в 1885 г. Г. Брунcom (H. Bruns) для обозначения функций, аналогичных характеристическим функциям среды, однако в дальнейшем он стал применяться в более широком смысле [48].
} 
С основами геометрической оптики можно ознакомиться в монографиях М. Борна, Э. Вольфа [48], Дж. Бруса, П. Джиблина [77] и Р. Фейнмана, Р. Лейтона, М. Сэндса [101], Ю. А. Кравцова, Ю. И. Орлова [149], В. И. Арнольда [28] и Т. Постона и И. Стюарта [180].

Цель этого раздела - проиллюстрировать работу геометрической теории приближения в задачах, связанных с уравнением эйконала.

Сразу же отметим, что в случае неоднородной среды, когда $n=n(x)$ не константа, лучи - это уже не прямые, а кривые линии, но тем не менее здесь возможно использовать аналогичный инструментарий геометрической теории приближений.

Под решением уравнения эйконала могут пониматься как классические, так и обобщенные решения (см. [150], [199]). В данной работе мы рассматриваем классические решения уравнения эйконала.

Пусть $\Omega$ - открытое подмножество пространства $\mathbb{R}^{n}$. Рассмотрим задачу нахождения классических решений простейшего уравнения эйконала

$$
|\nabla f(x)|=1, \quad x \in \Omega,
$$

где $f \in C^{1}(\Omega)$. Уравнение (1.2) описывает распространение световых лучей в однородной и изотропной среде, в геометрооптическом приближении. Краткая постановка данной задачи с указанием ряда простейших областей, для которых (1.2) имеет решение, приводится в [121; проблема 8].

Для изучения этой задачи важным оказывается знание геометрии поверхности уровня решения:

$$
L_{\theta}=\{x \in \Omega \mid u(x)=\theta\} .
$$

В нашем рассмотрении - это поверхность гладкости $C^{1}$, а если $u \in C^{k}$, то поверхность имеет гладкость $C^{k}$. Хорошо известно (см., например, [100]), что градиент эйконала $u(x)$ ортогонален поверхности уровня $L_{\theta}$ в точке $x$. Соответственно, поскольку потенциальное векторное поле $\mathbf{a}=\nabla u$ непрерывно на $\Omega$, то

$$
\int_{\gamma_{1}}(\mathbf{a}, d \mathbf{r})=\int_{\gamma_{2}}(\mathbf{a}, d \mathbf{r})
$$

для любых спрямляемых кривых $\gamma_{1}, \gamma_{2}$, содержащихся в односвязной подобласти $\Omega$, выходящих из одной и той же произвольной точки $x_{0} \notin L_{\theta}$ и заканчивающихся на одной и той же поверхности уровня.

Поскольку $|\mathbf{a}|=1$, то для любой кривой $\gamma$, выходящей из $x_{0}$, имеем

$$
\theta-\theta_{0}=\int_{\gamma}(\mathbf{a}, d \mathbf{r})
$$

и

$$
\left|\theta-\theta_{0}\right|=\left|\int_{\gamma}(\mathbf{a}, d \mathbf{r})\right| \leqslant \int_{\gamma}|\mathbf{a}||d \mathbf{r}|=|\gamma|,
$$

где $u\left(x_{0}\right)=\theta_{0}$. В силу соображений компактности величина $\theta-\theta_{0}$ достигается на некоторой интегральной кривой $\gamma_{0}$, в каждой точке которой касательный вектор совпадает с вектором градиента (или ему противоположным):

$$
\theta-\theta_{0}= \pm \int_{\gamma_{0}}(\nabla u, d \mathbf{r})= \pm \int_{\gamma_{0}} d s= \pm\left|\gamma_{0}\right|
$$

здесь $s$ - натуральная параметризация кривой $\gamma$. 
Теперь возьмем какую-нибудь точку $x_{0}$ из $\Omega$ и рассмотрим отрезок-кривую $I$ из $\Omega$, соединяющую точку $x_{0}$ и некоторую ближайшую точку из $L_{\theta}$. Длина этого отрезка - это расстояние до множества $L_{\theta}$. Поэтому длина кривой $\gamma$, соединяющей $L_{\theta}$ и $x_{0}$, не меньше длины отрезка $I$, и, следовательно, $|I|=\left|\gamma_{0}\right|$. Если из $x_{0}$ исходит путь $r=r(\cdot)$ кривой $\gamma$, для которого при некотором значении параметра $s$ касательный вектор отличен от $\mathbf{a}(r(s))$, то неравенство в $(1.5)$ будет строгим и, следовательно, длина кривой $\gamma$ будет больше длины отрезка $I$.

Подводя итог, получим, что $\left|\theta-\theta_{0}\right|=\left|\gamma_{0}\right|=|I|$ равно расстоянию от $x_{0}$ до линии уровня. Согласно известным результатам геометрической теории приближений (см. [32]) это означает, что ближайшая точка $x_{0}$ единственна и, значит, отрезок $I$ также единственен и является интегральной кривой.

Отсюда следует, что в $\Omega \subset \mathbb{R}^{n}$ поверхность уровня является “локально чебышёвским" множеством (с непрерывной метрической проекцией) и, следовательно, “локально солнечным" множеством. Дадим формальное определение через свойство регулярности.

Для $M \subset X$, где $X$ - линейное нормированное пространство, обозначим

$$
T_{M}:=\left\{x \mid \operatorname{card} P_{M} x=1\right\}
$$

(если $T_{M}=X$, то $M$ - чебышёвское множество). Точка $x_{0} \in \mathbb{R}^{n} \backslash M$ называется регулярной точкой, если существует окрестность $O\left(x_{0}\right)$ точки $x_{0}$, содержащаяся в $T_{M}$ (если $X=\mathbb{R}^{n}$, то это влечет непрерывность однозначного оператора метрической проекции $P_{M}$ на множестве $O\left(x_{0}\right)$, в бесконечномерном случае это полагается по определению). Для замкнутых множеств $M$ к особым точкам отнесем нерегулярные точки из $\mathbb{R}^{n} \backslash M$ и точки из $\overline{\operatorname{int}} M$. Особое множество для замкнутого $M$ - это множество всех точек, являющихся для него особыми.

Следует отметить, что особое множество есть объединение замыкания множества точек неединственности и замыкания внутренности множества $M$, а множество регулярных точек открыто. Надо сказать, что структуре особого множества в геометрической теории приближений также уделяется внимание (см., например, [32], [30], [152]). Не останавливаясь на этом вопросе подробно, отметим, что если у $C^{1}$-гиперповерхности в $\mathbb{R}^{n}$ особое множество пусто, то эта гиперповерхность является гиперплоскостью, если особое множество - точка, то гиперповерхность является сферой, а если особое множество - подпространство (размерности $k, 1 \leqslant k \leqslant n-2$ ), то "сферическим цилиндром" с образующими, являющимися сдвигами подпространства. Кроме того, отметим, что не существует $C^{1}$-гиперповерхности в $\mathbb{R}^{n}$, для которой особое множество есть гиперплоскость. Последний результат также получается применением геометрической теории приближений.

Стоит еще упомянуть, что на гладком компактном многообразии нет $C^{1}$-решений уравнения эйконала. При этом регулярные точки - это точки, в некоторых окрестностях которых расстояние до множества реализуется на единственных геодезических кривых. В этой связи отметим, что М. И. Карлов [131] рассмотрел на эллипсоиде нетривиальное чебышёвское множество и описал особое множество, на котором происходит переключение геодезических. По поводу некоторых вопросов геометрической оптики на многообразиях мы отсылаем читателя к обзору А. А. Аграчева и Ж. П. А. Готье [1]. 
1.1. Структура особого множества на плоскости. Рассмотрим вопрос о структуре особого множества на плоскости. Этот вопрос важен при решении уравнения эйконала.

Пусть $M$ есть $C^{2}$-многообразие в $\mathbb{R}^{2}$ с замкнутым следом. Изучим его особое множество $E$.

Особую точку $x$ множества $M$ назовем вершиной, если существует ближайшая для $x$ точка $y \in M$, для которой $x$ является центром кривизны, т. е. $\varrho(x, M)$ (расстояние от $x$ до $M$ ) есть радиус кривизны в точке $y$. Узлом назовем особую точку $x$, которая не является вершиной и для которой количество ближайших больше двух. Число компонент связности множества ближайших элементов узла назовем его кратностъю.

Из результатов работы [175] (см. также [138]) вытекает, что множество узлов для $M$ не более чем счетно. Отметим также, что дополнение до особого множества $E$ множества всех вершин и узлов $M$ состоит из точек, у которых в $M$ имеются ровно две ближайшие.

Гладким путем будем называть такое отображение $r: I \rightarrow \mathbb{R}^{2}(I \subset \mathbb{R}-$ промежуток), что $|\dot{r}| \neq 0$ на $I$. Если некоторый конец $a$ промежутка $I$ принадлежит этому промежутку, то точку $A=r(a)$ назовем кониом пути $r$. Будем говорить, что гладкий путь $r: I \rightarrow \mathbb{R}^{2}$ принадлежит классу $H_{0}^{2}$ (является $H_{0}^{2}$-путем), если отображение $\dot{r}$ липшицево на внутренности промежутка $I$ (т. е. на $\stackrel{\circ}{I}=\operatorname{int} I)$. Будем говорить, что путь $r$ в конце $A=r(a)$ имеет гладкость $C^{1}$ (соответственно $\left.H^{2}\right)$, если $r \in C^{1}(a)(\dot{r}-$ липшицево в точке $a$ ).

Далее будем рассматривать пути только трех типов. К первому типу отнесем простые пути $r:(a, b) \rightarrow \mathbb{R}^{2}$, для которых $r(t) \rightarrow \infty$ при $t \rightarrow a$ и $t \rightarrow b$. Такие пути будем называть неограниченными. Ко второму типу отнесем простые полуограниченные пути $r:[a, b) \rightarrow \mathbb{R}^{2}$, для которых $r(t) \rightarrow \infty$ при $t \rightarrow b$. $\mathrm{K}$ третьему типу отнесем простые ограниченные пути $r:[a, b] \rightarrow \mathbb{R}^{2}$.

Теорема 1.1. Рассмотрим $C^{2}$-многообразие на плоскости с замкнутым следом $M$. Тогда особое множество $Е$ для множества $M$ является замкнутым множеством, не пересекающимся с $М$. При этом:

1) множество неизолированных вершин $E$ образует нигде не плотное в $E$ подмножество, а множество изолированных вершин не более чем счетно и для каждой из них множество ее ближайших точек в $M$ образует окружность;

2) множество узлов - не более чем счетное множество, являющееся нигде не плотным в $E$;

3) дополнение множества всех вершин и узлов до Е представляет собой не более чем счетное множество следов простых $H_{0}^{2}$-путей за вычетом кониов; при этом кониы ограниченных и полуограниченных путей являются либо вершинами, либо узлами; если конеи является вершиной, то в этой точке путь имеет гладкость $C^{1}$, если же конеи, является узлом, то в нем путь имеет гладкость $H^{2}$; касательные векторы в каждой точке $T=r(t)(t \in \stackrel{\circ}{I})$ каждого пути $r(\cdot)$ параллельны биссектрисе угла $Y_{1} T Y_{2}$, где $Y_{1}$ и $Y_{2}$ - ближайшие $\kappa T$ для $M$, и промежутки $\left[Y_{1}, T\right)$ u $\left(T, Y_{2}\right]$ не пересекаются с особым множеством; 
4) из каждого узла исходят гладкие пути (у которых узел является кониом), число которых равно кратности узла.

Силъно регулярной точкой множества $M$ назовем такую регулярную точку этого множества, для которой локальный минимум функции расстояния $\rho_{x}(\cdot)=|x-\cdot|$ на множестве $M$ совпадает с глобальным (единственным).

Множество всех строго регулярных точек назовем строго регулярным множеством. Отметим, что для $C^{2}$-многообразия с замкнутым следом граница строго регулярного множества состоит из точек эволюты (геометрического места центров кривизны каких-то участков многообразия). В геометрической оптике эволюту также называют каустикой. Слабо особъми точками назовем точки дополнения $\mathbb{R}^{n} \backslash M$, не являющиеся сильно регулярными точками, а также точки из замыкания внутренности $M$. Слабо особое множество, как множество всех слабо особых точек, содержит всю каустику любого $C^{2}$-многообразия.

Возьмем четверть эллипса (с концами или без). Каустика такого множества является частью слабо особого множества и представляет собой четверть каустики всего эллипса (см. рис. 1).

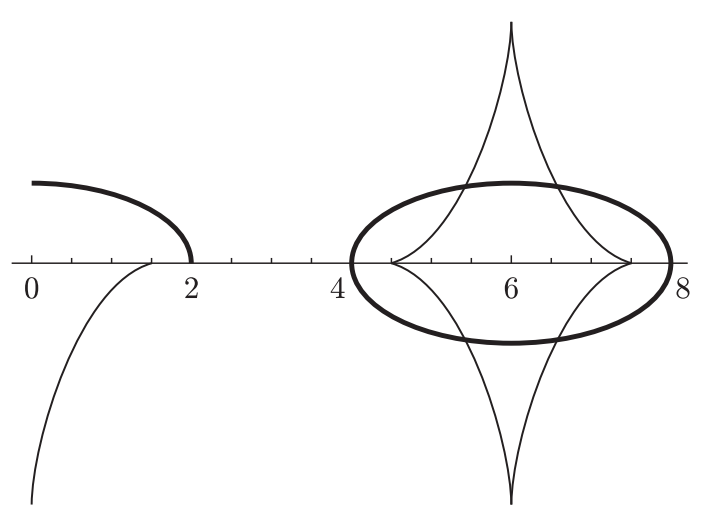

Рис. 1. Каустика для эллипса и четверти эллипса

Иногда для решения задач геометрической теории приближения удобно провести классификацию локальных экстремумов. Вообще, количество значений локальных минимумов функции $\rho_{x}(\cdot)$ для каждой точки $x$ не более чем счетно. Это позволяет упорядочить значения минимумов по возрастанию и назвать локальный минимум минимумом ранга $n$, если его значение стоит на $n$-м месте. Регулярными точками ранга $n$ назовем точки, у которых некоторые окрестности имеют единственные минимумы ранга $n$. Регулярные точки 1-го ранга это обычные регулярные точки. Особыми точками ранга $n(n \geqslant 2)$ назовем дополнение множества всех регулярных точек ранга $n$.

Для эллипса на плоскости внешность каустики состоит из регулярных точек ранга 1, у которых нет минимумов большего ранга (т. е. из сильно регулярных точек). Внутренность каустики, кроме точек большой оси, состоит из регулярных точек одновременно 1-го и 2-го рангов. Часть большой оси внутри каустики состоит из особых точек 1-го ранга. Вся каустика состоит из особых 
точек 2-го рода. При этом центры наибольшей кривизны эллипса (две вершины каустики) являются еще и особыми точками 1-го рода, а оставшиеся точки каустики - регулярными точками 1-го рода.

Пусть $N$ - открытое выпуклое множество в $\mathbb{R}^{n}$, а $M$ - произвольное его подмножество, замкнутое относительно $N$. Если существует открытый шар с центром в некоторой точке из $N$, пересечение которого с $M$ несвязно, то в $N$ найдется кусок липшицевой гиперповерхности, состоящей из точек неединственности (для этих точек число ближайших в $M$ не меньше двух). В случае,

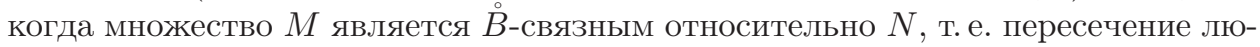
бого открытого шара с центром в произвольной точке из $N$ с множеством $M$ связно, можно описать структуру его особого множества. Не вдаваясь в подробности, отметим только один факт: если в $N$ есть точки неединственности, то там же найдется точка, у которой множество ближайших представляет собой сферу размерности $\geqslant 1$. Как и обещали, ограничимся только плоским случаем.

Опишем структуру регулярного и особого множеств для $M$ в множестве $N$ на плоскости, где $M$ есть $\stackrel{\circ}{B}$-связный относительно $N$ след $C^{1}$-многообразия. Оказывается, возможны только два варианта: либо все точки $N$ сильно регулярны для $M$, либо $M$ - это окружность в $N$ и ее центр - единственная особая точка (представляющая собой вырожденную каустику).

1.2. $C^{1}$-решения уравнения эйконала. Связь с солнечностью. Из известных результатов геометрической теории приближений (см. [222]), опирающихся на принцип неподвижной точки Брауэра, вытекает, что локалъно (на некоторой окрестности $O\left(x_{0}\right)$ регулярной точки $\left.x_{0}\right)$ выполняется свойство локальной солнечности, т. е. для любого $x \in O\left(x_{0}\right) \backslash L_{\theta}$ существует (единственная ближайшая) точка $y$ из $L_{\theta}$ такая, что $y$ будет являться ближайшей в $L_{\theta}$ для всех точек из пересечения $O\left(x_{0}\right)$ и луча $\ell_{y}$, выходящего из $y$ и проходящего через $x$. Хорошо известно (см., например, [100]), что это влечет непрерывную дифференцируемость функции расстояния $\rho\left(x, L_{\theta}\right)$ от $x$ до множества $L_{\theta}$ в точках регулярности, не лежащих в $L_{\theta}$; более того, принадлежность функции $\rho\left(\cdot, L_{\theta}\right)$ классу $C^{1}(x)$ равносильна регулярности в точке $x$.

Отметим следующий простой, но важный факт. Если $x_{0}$ - регулярная точка для множества $M$ (пусть $y$ - единственная ближайшая из $M$ для $x_{0}$ ) и если $x \in\left[x_{0}, y\right)$, то $y$ - единственная ближайшая для $x$. Это, в частности, дает, что

$$
\stackrel{\circ}{B}(x, \rho(x, M)) \cap M=\varnothing,
$$

где $\stackrel{\circ}{B}(x, r)$ - открытый шар с центром $x$ и радиусом $r$.

Заметим также, что для любого множества $M \subset \mathbb{R}^{n}$ функция $\rho(\cdot, M)$ совпадает с функцией $\rho(\cdot, \bar{M})$. Поэтому для нахождения решения задачи эйконала можно везде далее считать, что множество $M$ замкнуто.

Из сказанного выше вытекает, что $C^{1}$-решение $u(\cdot)$ уравнения эйконала $(1.2)$ локально представляется в виде $c \pm \rho(\cdot, M)$ для некоторого множества $M$. Отметим, что существуют односвязная область в $\mathbb{R}^{n}$ и $C^{1}$-решение уравнения эйконала, представляющее собой счетную склейку функций вида $c_{j} \pm \rho\left(\cdot, M_{j}\right)$, но не представимое в виде конечной склейки такого рода.

Основной вывод из этого таков (см., например, [32]): та часть $\Omega$, где решение имеет вид $c \pm \rho(x, M)$, состоит только из регулярных точек $M$. Следовательно, 
точки особого множества для $M$ обязаны содержаться в $\mathbb{R}^{n} \backslash \Omega$ или в других частях $\Omega$ (где решение не представляется в виде $c \pm \rho(\cdot, M)$ для данного $M$ ).

Таким образом, нами получены следующие утверждения.

Теорема 1.2. Пусть $и \in C^{1}(\Omega)$ - решение уравнения эйконала (1.2). Тогда или особая точка $x_{0}$ для поверхности уровня $L_{\theta}$ не лежит в $\Omega$, или в некоторой окрестности $O\left(x_{0}\right)$ функция $u(\cdot)$ не представляется в виде $c \pm \rho\left(\cdot, L_{\theta}\right)$.

Теорема 1.3. Функиия $c \pm \rho(x, M)$ является $C^{1}$-гладким решением уравнения эйконала (1.2) на области $\Omega$, если в $\Omega$ нет особых точек $M$ и $M \cap \Omega=\varnothing$.

СледСтвиЕ 1.1. Если $M \subset \mathbb{R}^{n} \backslash \Omega$ - выпуклое множество, то функиии

$$
c \pm \rho(x, M)
$$

являются $C^{1}$-гладкими решениями уравнения эйконала (1.2) на $\Omega$.

Следующий результат установлен другим методом в [121; с. 266].

ТЕОРема 1.4. $C^{1}$-гладкими решениями уравнения эйконала $(1.2)$ на $\Omega=\mathbb{R}^{n}$ являются в точности афбинные функиии $c \pm x^{*}(\cdot)$, где $\left|x^{*}\right|=1, x^{*}(\cdot)=$ $\left(x^{*}, \cdot\right)$.

Как следствие, аффинные функции $c \pm x^{*}(\cdot),\left|x^{*}\right|=1$, дают решение уравнения (1.2) на любом открытом подмножестве $\Omega \subset \mathbb{R}^{n}$.

Следующий результат достаточно очевиден и вытекает из того, что функция расстояния до множества $\rho(\cdot, M)$ локально является решением в точках регулярности.

ТеОрема 1.5. Пусть $\Omega$ - область в $\mathbb{R}^{n}$, и пусть $M \subset \Omega$ есть $C^{1}$-гладкая гиперповерхность, разделяющая $\Omega$ на две компоненты связности $A_{1} u A_{2}$. Определим функиию

$$
\varphi(x)= \begin{cases}\rho(x, M), & x \in A_{1} ; \\ -\rho(x, M), & x \in A_{2} ; \\ 0, & x \in M .\end{cases}
$$

Предположим, что $\rho(\cdot, M)$ имеет гладкость $C^{1}$ на $\Omega \backslash M$ (т.е. все точки $\Omega$ регулярны). Тогда функции $c \pm \varphi(\cdot)$ являются $C^{1}$-гладкими решениями уравнения эйконала (1.2) на области $\Omega$.

ТеОрема 1.6. Пусть $\Omega \subset \mathbb{R}^{n}$ - область, $K:=\mathbb{R}^{n} \backslash \Omega$ - компакт. Тогда класс функиий $c \pm \rho(\cdot, M)$, где $M \subset K$, являющихся $C^{1}$-гладкими решениями уравнения эйконала (1.2) на области $\Omega$, совпадает с классом функиий $c \pm \rho(\cdot, M)$, где $M \subset K$ выпукло.

ДокАЗАтЕльство. Если $M$ выпукло, то $\bar{M}$ является чебышёвским множеством в $\mathbb{R}^{n}$ и, следовательно, $\mathbb{R}^{n} \backslash \bar{M}$ не содержит особых точек. Тогда по теореме 1.3 функции $c \pm \rho(\cdot, M)$ являются $C^{1}$-гладкими решениями уравнения $(1.2)$ на $\Omega$. Обратно, предположим, что $M$ не выпукло. Положим $M_{0}:=\operatorname{conv} M$. Если граница $M_{0}$ содержится в $M$, то $M_{0} \subset K$ в силу связности $\Omega$, и, значит,

$$
\rho(x, M)=\rho\left(x, M_{0}\right), \quad x \in \Omega .
$$


Если же граница $M_{0}$ не принадлежит $M$, то $M$ не обладает внешним чебышёвским слоем достаточно большой величины (см. [23]). Воспользовавшись характеризацией множеств с внешним чебышёвским слоем [23], получаем, что для любого $r>0$ имеются особые точки множества $M$, расположенные от $M$ на расстоянии $\geqslant r$, и, значит, при больших $r$ множество $\Omega$ всегда содержит особые точки для $M$. Поэтому $u$ не может быть $C^{1}$-решением. Теорема доказана.

Tеорема 1.7. Пусть $\Omega \subset \mathbb{R}^{n}$ - область, $K=\mathbb{R}^{n} \backslash \Omega$ - компакт. Тогда всякое $C^{1}$-решение уравнения эйконала представляется в виде функции $c \pm$ $\varphi(\cdot)$ из теоремы 1.5 для некоторого множества $M$, представляющего собой границу выпуклого тела, особое множество которого содержится в $K$.

Пример 1.1. Как уже отмечалось в теореме 1.4 для случая $\Omega=\mathbb{R}^{n}$, множество решений уравнения эйконала (1.2) на $\Omega=\mathbb{R}^{n}$ состоит в точности из аффинных функций вида $c \pm x^{*}(\cdot)$, где $\left|x^{*}\right|=1, x^{*}(\cdot)=\left(x^{*}, \cdot\right)$. Такие функции суть функции вида $c \pm \varphi(\cdot)$ из теоремы 1.5 в случае, когда $M$ - гиперплоскость; они к тому же являются линиями уровня решений $c \pm x^{*}(\cdot)$.

Эти решения также являются решениями на любой подобласти в $\mathbb{R}^{n}$.

Отметим, что все результаты примера 1.1 остаются в силе, если вместо $\mathbb{R}^{n}$ рассматривать произвольное гильбертово пространство. При этом следует воспользоваться классическим результатом Асплунда (см. [32]), согласно которому в гильбертовом пространстве чебышёвское множество с непрерывной метрической проекцией выпукло.

Пример 1.2. $\Omega=\mathbb{R}^{n} \backslash\left\{a_{1}\right\}$. Из теоремы 1.6 следует, что к решениям уравнения (1.2) из примера 1.1 добавляются решения вида $c \pm\left|x-a_{1}\right|$ (см. также [121; формула (8.2)]). На самом деле, исследуя эту задачу подробнее с использованием указанных выше теорем и леммы 2.2 обзора [32] можно показать, что других решений нет.

Пример 1.3. $\Omega=\mathbb{R}^{n} \backslash\left\{a_{1}, \ldots, a_{k}\right\}$. В этом случае весь класс $C^{1}$-решений уравнения эйконала на $\Omega$ состоит в точности из функций примера 1.1 и функций вида $c \pm\left|x-a_{j}\right|, j=1, \ldots, k$.

В примерах 1.1-1.3 предположение о $C^{1}$-гладкости решения уравнения эйконала приводит к гладкости $C^{\infty}$, а в примерах 1.4 и 1.5 - к гладкости $H^{2}$.

Пример 1.4. $\Omega=\mathbb{R}^{n} \backslash[a, b]$. Опишем класс всех $C^{1}$-решений уравнения эйконала на $\Omega$. Пусть $[c, d]$ - подотрезок $[a, b], r(x):[c, d] \rightarrow \mathbb{R}_{+}$- неотрицательная выпуклая вверх функция. Рассмотрим выпуклое множество

$$
D=\bigcup_{x \in[c, d]} B(x, r(x))
$$

и его границу $M$. Отметим, что особое множество для $M$ является выпуклым подмножеством отрезка $[c, d]$ (что вытекает, к примеру, из результатов Балаганского [30]). Аналогично теореме 1.5 рассмотрим функции

$$
\varphi(x)=c \pm \begin{cases}\rho(x, M), & x \in A_{1}, \\ -\rho(x, M), & x \in A_{2}, \\ 0, & x \in M,\end{cases}
$$


где $A_{1}$ и $A_{2}$ - соответственно внешность и внутренность $D$. Все эти функции в точности составляют класс всех $C^{1}$-решений уравнения эйконала на $\Omega$. В частности, сюда входят функции вида $c \pm \rho(x,[c, d])$.

Пример 1.5. $K:=\mathbb{R}^{n} \backslash \Omega$ - конечное объединение попарно непересекающихся отрезков $\left[a_{i}, b_{i}\right], i=1, \ldots, N$. Для каждого отрезка рассмотрим функции из примера 1.4. Все такие функции, построенные для каждого из отрезков $\left[a_{i}, b_{i}\right]$, в точности образуют класс всех $C^{1}$-решений уравнения эйконала на $\Omega$.

Отметим, что для открытых множеств представляет особый интерес также нахождение решений уравнения эйконала из класса $C^{1}(\Omega) \cap C(\bar{\Omega})$.

\section{2. Обзор основных понятий и определений геометрической теории приближений}

В XIX в. возникла необходимость решения важной практической задачи, относящейся к проблемам усовершенствования конструкции паровой машины - знаменитого параллелограмма Уатта, использовавшегося для превращения прямолинейного движения поршня во вращательное движение коромысла паровой машины. Схема паровой машины сейчас кажется удивительно простой, однако понадобились усилия многих инженеров, прежде чем эта схема обрела свой окончательный вид. Именно в связи с этой задачей в середине XIX в. П.Л. Чебышёв ввел в науку важное понятие наилучшего приближения (а именно, наилучшего приближения относительно равномерной нормы) и систематически применял его в приложениях. В дальнейшем понятия величины и элемента наилучшего приближения были перенесены на случай общих линейных нормированных пространств и стали исходным пунктом геометрической теории приближений.

Величиной наилучшего приближения или расстоянием от заданного элемента $x$ линейного нормированного пространства $X$ до заданного непустого множества $M \subset X$ называется величина

$$
\rho(x, M):=\inf _{y \in M}\|x-y\| .
$$

Понятия и свойства, определяемые в терминах наилучшего приближения, в частности свойства существования, единственности, устойчивости элементов наилучшего приближения, называются аппроксимативными. Прежде всего, таким является понятие элемента наилучшего приближения, или ближайшей точки. Это есть (для заданного $x \in X$ ) такая точка $y \in M$, для которой $\|x-y\|=\rho(x, M)$. Множество всех ближайших точек (элементов наилучшего приближения, или, кратко, наилучших приближений) в $M$ для заданного $x$ обозначается $P_{M} x$. Иными словами,

$$
P_{M} x:=\{y \in M \mid \rho(x, M)=\|x-y\|\} .
$$

Ниже рассматриваются "солнечные" свойства подмножеств линейных нормированных пространств, представляющие собой аппроксимативно-геометрическую характеристику, а также исследуется связность и устойчивость множеств ближайших и почти ближайших элементов. 
"Солнца" обладают важными характеристическими признаками. Им присущи те или иные свойства отделимости: шар можно отделить от такого множества посредством шара сколь угодно большого радиуса или опорного конуса. Эти свойства стоят в одном ряду с известными свойствами отделимости выпуклых множеств посредством полупространств (гиперплоскостей), развитием которых является критерий Колмогорова наилучшего элемента.

Ниже мы будем рассматривать структурные характеристики солнц, наиболее важными из которых являются геометрически-топологические свойства (в частности, связность и выпуклость). При этом будут рассматриваться как прямые теоремы геометрической теории приближений, в которых из структурных характеристик множеств выводят их аппроксимативные свойства, так и обратные теоремы, в которых из аппроксимативных свойств множеств получают их структурные характеристики. В качестве аппроксимативных характеристик множеств будут рассматриваться свойства единственности, существования наилучшего приближения, чебышёвости, аппроксимативной компактности, солнечности, а также устойчивости наилучшего и почти наилучшего приближения. Под структурными характеристикам множеств обычно понимают свойства линейности, конечномерности, компактности, выпуклости, различной связности и гладкости этих множеств. Например, хорошо известно, что из компактности непустого множества следует, что оно является множеством существования (это - прямая теорема).

Обратные теоремы по отношению к приложениям выступают в следующей роли. Выяснив, что исследуемый объект не обладает "хорошими” структурными характеристиками, из этих теорем выводим, что он не обладает и "хорошими" аппроксимативными свойствами. Обычно таким путем удается установить, что данный объект не является множеством существования или единственности.

На сегодняшний день область применения геометрической теории приближений лежит в теории оптимального управления системами с распределенными параметрами (А. В. Фурсиков [106]-[108], М. В. Яшина [229], [230]), теории некорректных задач (В. К. Иванов, В.В.Васин, В. П. Танана [126], Ф. Фарачи, А. Ианидзотто [99], Л. Зайичек [231]), теории неоднозначной разрешимости нелинейных дифференциальных уравнений (И. Г. Царьков [209], [210], [212], [213], Б. Ричери [184], Ф. Фарачи, А. Ианидзотто [98] и др.), теории приближения функций (С. В. Конягин [140], А.Л. Гаркави [109], С. Я. Хавинсон [133], К. С. Рютин [186], [187], П. А. Бородин [51], [53] и др.), топологических минимаксных теоремах (Х. Кёниг [137], Б. Ричери [183]), теории критических точек в негладком случае (Д. Браесс [56], Б. Ричери [182]), теории обучения при построении оптимального оценщика (Ю.В. Малыхин [157]), при исследовании устойчивости по различным параметрам решений общих экстремальных задач и многозначных отображений (В. И. Бердышев [38]-[41], Ф. Дойч, Дж. М. Ламберт [88], А. В. Маринов [159]-[161], П. Шварцман [190], М. В. Балашов и Д. Реповш [34], К. В. Чеснокова [82] и др.), приближении ганкелевыми матрицами, а также в выпуклом анализе - к примеру, при исследовании функции Моро и связанного с ней свойства проксрегулярности множеств и функций, которое является локальным вариантом свойства проксимальной гладкости и играет 
важную роль (как в теоретическом, так и в вычислительном аспекте) в оптимизации, вариационном анализе, нелинейном анализе и задачах восстановления сигналов (Р. А. Поликвин и Р. Т. Рокафеллар [177], Ф. Бернард и Л. Тибо [46], Б. Ричери [184], М. В. Балашов, Г. Е. Иванов [33], А. Журани, Л. Тибо, Д. Загродны [130], В. Н. Соловьев [195] и др.). В связи с вышесказанным стоит также отметить обзор В. М. Тихомирова [200], в котором, в частности, подробно раскрывается роль геометрической теории приближений в задачах теории приближения функций, выпуклом анализе и других областях математики, а также обзоры [222], [32], [132], [141]. Выше отмечалась роль геометрической теории приближений в решении задач геометрической оптики (уравнение эйконала).

В данном обзоре мы почти не затрагиваем обширный вопрос о выпуклости чебышёвских множеств и их обобщений (подробнее об этом см. работы Л. П. Власова [222], В. С. Балаганского и Л.П. Власова [32], М.И. Карлова и И. Г. Царькова [132], Ф. Дойча [87], А. Л. Брауна [71], [72], И. Г. Царькова [205], [206], А.Р. Алимова [6], П.А. Бородина [52], Дж. Флетчера [102], Дж. Флетчера и У. Б. Моорса [103]).

Подчеркнем, что ниже мы рассматриваем классические вопросы геометрической теории приближений, не затрагивая ряд постановок, к примеру связанных с приближениями относительно расстояний Брегмана (X. Х. Баушке, К. Ванг, Ч. Ли, Дж. Йе, К. Юань [35] и др.), n-расстояний в смысле Бородина (П. А. Бородин [52], [50]) и несимметричных расстояний (А. Брондстед [62], А. Р. Алимов [5], [8], [13], П. А. Бородин [52], С. Кобзаш [85]), в том числе относительно неограниченных квазишаров (Г. Е. Иванов и М. С. Лопушански [125], [124]).

Всюду ниже $X$ - действительное линейное нормированное пространство. Случаи, когда $X$ - несимметрично нормированное пространство, будут оговорены особо. Далее:

$B(x, r)$ - замкнутый шар с центром $x$ и радиусом $r$;

$\stackrel{\circ}{B}(x, r)$ - открытый шар с центром $x$ и радиусом $r$;

$S(x, r)$ - сфера с центром $x$ и радиусом $r$.

В частном случае мы полагаем $B:=B(0,1)$ - единичный шар, $S=S(0,1)$ единичная сфера.

Множество $M$ называется множеством существования (или проксиминальным множеством), если для каждой точки $x$ множество $P_{M} x$ ее ближайших элементов непусто. Множество $M$ называется множеством единственности, если для каждой точки $x$ множество $P_{M} x$ пусто или одноточечно. Множество существования всегда замкнуто и непусто: действительно, если предельная точка $M$ не содержится в $M$, то вполне очевидно, что у нее нет ближайших в $M$. В конечномерном $X$ верно и обратное утверждение: любое замкнутое непустое множество является множеством существования.

Пусть $\varnothing \neq M \subset X$. Точка $x \in X \backslash M$ называется точкой солнечности, если существует точка $y \in P_{M} x \neq \varnothing$ (называемая точкой светимости) такая, что

$$
y \in P_{M}((1-\lambda) y+\lambda x) \quad \text { для всех } \lambda \geqslant 0
$$

(это геометрически означает, что из точки $y$ исходит "солнечный” луч, проходящий через $x$, для каждой точки которого точка $y$ является ближайшей из $M)$. 
Точка $x \in X \backslash M$ называется точкой строгой солнечности, если $P_{M} x \neq \varnothing$ и условие (2.1) выполнено для любой точки $y \in P_{M} x$. Если же для $x \in X \backslash M$ условие (2.1) выполнено для любой точки $y \in P_{M} x$, то точка $x$ называется $m о ч-$ кой строгой протосолнечности (при этом, в отличие от точки строгой солнечности, точка $y$, ближайшая к $x$, не обязана существовать).

Множество $M \subset X$ называется солнием (соответственно строгим солнцем), если каждая точка $x \in X \backslash M$ является точкой солнечности (соответственно строгой солнечности) для $M$. Множество $M \subset X$ называется строгим протосолнием, если каждая точка $x \in X \backslash M$ является точкой строгой протосолнечности (см. рис. 2). Множество $M \subset X$ называется протосолнием, если для любого $x \notin M$ в $M$ найдется точка светимости при условии, что $P_{M} x \neq \varnothing$.

Нам также потребуется локальный вариант определения солнечности. Пусть $M, G \subset X$. Множество $M$ называется солнцем относительно $G$, если для любой точки $x \in G$ найдется точка $y \in P_{M}$, являющаяся ближайшей для всех точек $z$ луча $(1-\lambda) y+\lambda x, \lambda \geqslant 0$, из (2.1) при условии, что $z \in G$.

Как правило, мы будем предполагать строгое протосолнце замкнутым. Отметим, что (замкнутое) строгое протосолнце не обязано быть множеством существования. Также отметим, что выпуклое множество всегда является строгим протосолнцем (выпуклое множество существования - строгим солнцем).

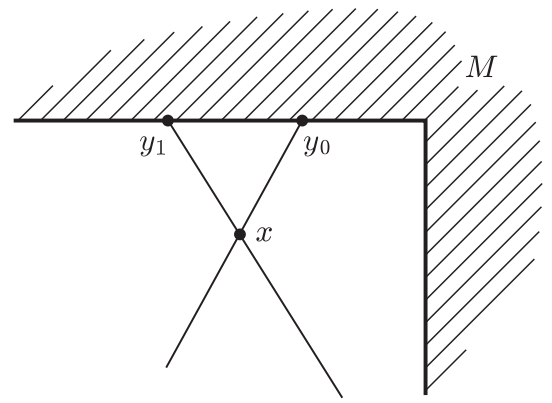

Рис. 2. Солнце $M$, не являющееся строгим солнцем в пространстве $\ell^{\infty}(2)$ (единичный шар - "квадратик"). Здесь $y_{0}$ - точка светимости для $x$; точка $y_{1}$ принадлежит $P_{M} x$, но не является точкой светимости.

Понятие "солнце" было введено Н. В. Ефимовым и С. Б. Стечкиным в [96]. Термин "строгое протосолнце" введен авторами в [25] во избежание путаницы в понятиях "строгое солнце существования" (строгое солнце) и "строгое солнце без предположения о существования ближайшего элемента" (строгое протосолнце), которые к тому же в некоторых школах обозначались просто термином "солнце". Отметим, что строгие протосолнца суть замкнутые множества Колмогорова, т. е. множества, на которых выполнен критерий Колмогорова ближайшего элемента (см. п. 3.2).

Поскольку в конечномерном пространстве $X$ замкнутое непустое множество является множеством существования, то в таких $X$ всякое строгое протосолнце 
является строгим солнцем. В бесконечномерном случае это уже не так - строгое протосолнце может быть антипроксиминальным, т. е. любая точка вне его вообще не имеет ближайших в множестве.

Отдавая дань уважения П. Л. Чебышёву как основателю теории приближений, Н. В. Ефимов и С.Б. Стечкин [96] предложили новый термин "чебышёвское множество", который практически сразу стал общепринятым - до (а иногда и после) Ефимова и Стечкина иными вариантами термина "чебышёвское множество" были EU-множество, множество Хаара или множество Моцкина. Итак, множество $M$ называется чебышёвским, если для каждого $x$ элемент наилучшего приближения из $M$ существует и единственен (иными словами, чебышёвские множества суть в точности множества существования и единственности). Если чебышёвское множество является солнцем, то говорят, что оно чебышёвское солнце. П.Л. Чебышёв ввел в науку важное понятие наилучшего приближения, в частности равномерного наилучшего приближения, систематически применял его в приложениях и разработал его теоретические основы. При изучении вопроса наилучшего приближения в $C[a, b]$ множествами $\mathscr{P}_{n}$ многочленов степени не выше $n$ и $\mathscr{R}_{m, n}$ дробно-рациональных функций,

$$
\mathscr{R}_{m, n}:=\left\{\frac{p}{q} \mid p \in \mathscr{P}_{n}, q \in \mathscr{P}_{m}, q \neq 0\right\}
$$

на $[a, b]$, в работах Чебышёва и его учеников родилось новое понятие альтернанса (сам термин "альтернанс" был введен позднее Н. И. Ахиезером). В дальнейшем, развивая идеи Чебышёва об альтернансе, П. Кирхбергер, Э. Борель, Дж. В. Юнг (для многочленов) и Н. И. Ахиезер и Дж. Уолш (для дробно-рациональных функций) обосновали единственность наилучших приближений и установили теорему существования наилучшего приближения. По поводу вопросов, связанных с историей возникновения теории приближений, мы отсылаем читателя к монографиям А. А. Гусака [119], К.-Г. Штеффенса [198] и работам В. Л. Гончарова [111], [112], А. А. Гусака [118], В. М. Тихомирова [200], П. Бутцера и $Ф$. Йонгманса [79].

Таким образом, множества $\mathscr{R}_{m, n}$ и $\mathscr{P}_{n}$ являются чебышёвскими множествами в $C[a, b]$. При этом оказалось, что элементы наилучшего равномерного приближения из $\mathscr{R}_{m, n}$ и $\mathscr{P}_{n}$ характеризуются в терминах альтернанса. Из этой характеризации вытекает (см. п. 3.2$)$, что множество $\mathscr{R}_{m, n}$ является чебышёвским солнцем в $C[0,1]$.

Множество обобщенно-рациональных функций

$$
\mathscr{R}_{V, W}=\left\{\frac{p}{q} \mid p \in V, q \in W, q(t)>0, t \in Q\right\}
$$

является строгим протосолнцем в $C(Q)$; здесь $V, W$ - произвольные выпуклые подмножества в действительном или комплексном $C(Q), Q$ - хаусдорфов компакт (Б. Брозовский и Р. Вегман [70], Л. Чонг и Дж. А. Ватсон [83], Д. Браесс [57]). В отличие от классического случая $\mathscr{R}_{m, n}$ при приближении обобщенными дробями $\mathscr{R}_{V, W}$ наилучшее приближение может не существовать или не быть единственным. 
Напомним еще ряд определений.

Непустое замкнутое множество $M$ называется:

$\alpha$-солнцем, если для любой точки $x \notin M$ существует луч $\ell$ с вершиной $x$ такой, что для любого $z \in \ell$ имеет место равенство

$$
\rho(z, M)=\|z-x\|+\rho(x, M) .
$$

Всякое солнце является $\alpha$-солнцем. Отметим, что (в отличие от солнц) $\alpha$-солнце может иметь собственные изолированные точки (примером является "двоеточие" $M:=\{(0,0)\} \cup\{(1,1)\}$ на плоскости с максимум-нормой);

$\beta$-солнцем, если для любых $x \notin M$ и $r>0$ существует $z$, для которого

$$
\rho(z, M)-\rho(x, M)=\|z-x\|=r ;
$$

$\gamma$-солнием, если для любых $x \notin M$ и $r>0$ существует последовательность $\left(z_{n}\right)$ такая, что

$$
\rho\left(z_{n}, M\right)-\rho(x, M) \rightarrow r, \quad\left\|z_{n}-x\right\|=r ;
$$

отношение " $M$ является $\gamma$-солнцем" эквивалентно отношению "для всякого $V=$ $B(x, R), x \notin M$, выполнено равенство $\sup \{\rho(u, M) \mid u \in V\}=R+\rho(x, M)$ ";

$\delta$-солнием, если для любой точки $x \notin M$ найдется последовательность $z_{n} \rightarrow x$ такая, что

$$
\lim _{n \rightarrow \infty} \frac{\rho\left(z_{n}, M\right)-\rho(x, M)}{\left\|z_{n}-x\right\|} \rightarrow 1 .
$$

Понятие $\alpha$-солнца введено Л. П. Власовым в [218]; он же [221] ввел понятия $\gamma$ - и $\delta$-солнц.

Каждое $\alpha$-солнце является $\beta$-солнцем, а $\beta$-солнце - $\gamma$-солнцем. Отметим, что строгое протосолнце может не быть $\beta$-солнцем (см. пример 4.2). В конечномерном пространстве классы $\alpha$-, $\delta$ - и $\gamma$-солнц совпадают. В банаховом пространстве классы $\delta$ - и $\gamma$-солнц равны [222; теорема 3.3].

В работе В. Брекнера [58] (см. также [59]) вводится понятие "солнце относительно сублинейного функционала" (в современных терминах - "солнце относительно несимметричной нормы"), что можно рассматривать как перенесение солнечности на общие экстремальные задачи, возникающие, в частности, в теории приближений относительно несимметричных расстояний.

Некоторые соотношения между классами “солнц" будут рассмотрены в разделе 4.

Стоит отметить, что замкнутые $r$-окрестности $(r>0)$ солнц являются солнцами. Именно, для $M \subset X$ рассмотрим

$$
M_{r}:=\{x \in X \mid \rho(x, M) \leqslant r\} .
$$

Ефимов и Стечкин [96] показали, что если $\rho(x, M) \geqslant r$, то

$$
\rho(x, M)=\rho\left(x, M_{r}\right)+r .
$$

Отсюда вытекает, что если $M$ - солнце, то $M_{r}$ также является солнцем (см. [222; гл. III, §2]), а если $M-$ множество существования, то $M_{r}$ - также проксиминально и, аналогично, если $M$ - строгое солнце ( $\delta$-солнце), то $M_{r}$ также обладает соответствующим свойством. Это перекликается со свойствами решений 
уравнения эйконала, где поверхности уровня локально являются границами окрестностей предыдущих поверхностей уровня.

Следуя Л.П. Власову, если Q обозначает некоторое свойство (например, "связность"), мы будем говорить, что замкнутое множество $M$ обладает свойством

$P$-Q, если при всех $x \in X$ множество $P_{M} x$ непусто и обладает свойством $\mathrm{Q}$;

$P_{0}$ Q , если $P_{M} x$ обладает свойством Q при всех $x \in X$;

$B$-Q, если $M \cap B(x, r)$ обладает свойством Q при всех $x \in X, r>0$;

$\stackrel{\circ}{B}-\mathrm{Q}$, если $M \cap \stackrel{\circ}{B}(x, r)$ обладает свойством $\mathrm{Q}$ при всех $x \in X, r>0$.

$\mathrm{K}$ примеру, замкнутое подмножество конечномерного пространства $P$-непусто, т. е. является множеством существования (проксиминально).

ЗАмЕчание 2.1. B-связные множества иногда называют $V$-связными (здесь буква "V" безусловно связана с Л.П. Власовым, который обозначал шары как $V(x, r))$. Наш термин " $B$-связность" согласуется с более привычным обозначением шаров как $B(x, r)$, а также с понятием "ограниченная связность" (bounded connectedness), введенным Д. Вулбертом в 1960-х годах.

Множество называется ограниченно компактным, если его пересечение с любым замкнутым шаром компактно.

Точка $x \in X$ называется точкой аппроксимативной компактности для множества $M(x \in \mathrm{AC}(M))$, если из любой последовательности $\left(y_{n}\right)_{n \in \mathbb{N}} \subset M$, удовлетворяющей соотношению $\left\|x-y_{n}\right\| \rightarrow \rho(x, M)$ (такая последовательность называется минимизирующей), можно выбрать подпоследовательность, сходящуюся к некоторой точке из $M$. Нетрудно проверить, что каждая точка аппроксимативной компактности $x \in X$ является точкой существования (т. е. $\left.P_{M} x \neq \varnothing\right)$. Множество $M \subset X$ называется аппроксимативно компактным, если каждая точка $x \in X$ является точкой аппроксимативной компактности для $M$. Понятие аппроксимативно компактного множества было введено Ефимовым и Стечкиным в [97]. Ясно, что ограниченно компактное множество аппроксимативно компактно.

$\mathrm{K}$ примеру, в $L^{p}, 1<p<\infty$, множество $\mathscr{R}_{m, n}$ дробно-рациональных функций аппроксимативно компактно, но не ограниченно компактно [97]. Однако в пространстве $C[0,1]$ множество $\mathscr{R}_{m, n}$ уже не аппроксимативно компактно. Действительно, с одной стороны, известно (Х. Мэли и К. Вицгаль [156], а также Д. Браесс [56]), что метрическая проекция на (чебышёвское множество) $\mathscr{R}_{m, n}$ имеет точки разрыва. С другой стороны, метрическая проекция на любое аппроксимативно компактное чебышёвское множество непрерывна (см., например, [222; следствие 2.2]).

Кратко касаясь общего вопроса о связи между классами аппроксимативно и ограниченно компактных множеств, отметим следующий результат: в любом бесконечномерном слабо компактно порожденном банаховом пространстве (т.е. в пространстве $X$, в котором существует слабо компактное множество, линейная оболочка которого плотна в $X$ ) и, в частности, в любом сепарабельном банаховом пространстве существует ограниченное аппроксимативно компактное, но не локально компактное (не ограниченно компактное) множество (П. А. Бородин [49], [54]). 
В работе рассматриваются следующие классы пространств.

(B) - класс банаховых пространств;

(Rf) - класс рефлексивных пространств;

(R) - класс строго выпуклых пространств (т. е. пространств, единичная сфера которых не содержит невырожденных отрезков);

(S) - класс гладких пространств (в каждой точке $x \in S$ существует единственная опорная гиперплоскость); иными словами, $X \in(S)$, если норма пространства $X$ дифференцируема по Гато во всех точках $x \neq 0$;

(SS) - класс сильно гладких пространств, т. е. таких $X \in(\mathrm{B})$, что для любой точки $x \in S$ и $f \in S^{*}$ такой, что $f(x)=1$, и для любых последовательностей $\left(x_{n}\right) \subset X$ и $\left(r_{n}\right) \subset \mathbb{R}$ таких, что $r_{n} \rightarrow \infty$ и $x \notin B\left(x_{n}, r_{n}\right) \supset B(0,1-1 / n), n \in \mathbb{N}$, верно включение

$$
\left\{y \in X \mid f^{*}(y)<1\right\} \subset \bigcup_{n \in \mathbb{N}} B\left(x_{n}, r_{n}\right) ;
$$

класс (SS) был введен Власовым [219]; он включает в себя локально равномерно выпуклые гладкие пространства и пространства, равномерно гладкие по каждому направлению;

(UR) - класс равномерно выпуклых банаховых пространств, т. е. таких, что для каждого $\varepsilon \in(0,2]$ существует $\delta>0$ такое, что если $x, y \in X,\|x\|=\|y\|=1$ и $\|x-y\| \geqslant \varepsilon$, то

$$
\left\|\frac{x+y}{2}\right\| \leqslant 1-\delta
$$

(LUR) - класс локально равномерно выпуклых пространств, т. е. таких банаховых пространств $X$, что для любых точек $x, x_{1}, x_{2}, \ldots \in X$ условие

$$
\|x\|=\left\|x_{1}\right\|=\left\|x_{2}\right\|=\cdots=\lim \left\|\frac{x+x_{n}}{2}\right\|, \quad n \rightarrow \infty,
$$

влечет сходимость $\left(x_{n}\right)$ к $x$;

(CLUR) - класс пространств, обладающих свойством: из соотношений $x \in S$, $y_{n} \in S,\left\|x+y_{n}\right\| / 2 \rightarrow 1$ следует существование сходящейся подпоследовательности у $\left(y_{n}\right)$;

(ES) - класс пространств Ефимова-Стечкина (такие пространства характеризуются тем, что в них всякая гиперплоскость аппроксимативно компактна).

Согласно классической теореме Джеймса

$$
(\mathrm{ES}) \subset(\mathrm{Rf})
$$

Также отметим, что

$$
\begin{gathered}
(\mathrm{UR}) \varsubsetneqq(\mathrm{ES}), \quad(\mathrm{ES}) \not \subset(\mathrm{CLUR}), \\
(\mathrm{LUR}) \cap(\mathrm{Rf}) \subset(\mathrm{ES}) \cap(\mathrm{R}), \quad(\mathrm{CLUR}) \cap(\mathrm{Rf}) \subset(\mathrm{ES})
\end{gathered}
$$

(см. [192], [32]). Иногда пространство Ефимова-Стечкина называют рефлексивным пространством со свойством Кадеца-Кли (или свойством Радона-Рисса). Термин "пространство Ефимова-Стечкина" был введен И. Зингером [192] 
в знак признания особых заслуг Н. В. Ефимова и С. Б. Стечкина в становлении геометрической теории приближений.

Существует много характеризаций пространств Ефимова-Стечкина (см. [142], [139], [84], [163], [32]). Из последних работ отметим [2]: пространство $X$ является пространством Ефимова-Стечкина тогда и только тогда, когда $X$ рефлексивно, любое замкнутое выпуклое подмножество единичной сферы $S$ компактно и любая достижимая грань шара $B$ является строго достижимой.

Отметим следующие включения:

$$
\begin{gathered}
(\mathrm{UR}) \subset(\mathrm{LUR}) \subset(\mathrm{CLUR}), \\
(\mathrm{LUR}) \subset(\mathrm{R}), \quad(\mathrm{S}) \cap(\mathrm{LUR}) \subset(\mathrm{SS}) \subset(\mathrm{S}), \quad(\mathrm{ES}) \subset(\mathrm{UR}), \\
(\mathrm{UR}) \subset(\mathrm{Rf}) \cap(\mathrm{LUR}) \subset(\mathrm{Rf}) \cap(\mathrm{CLUR}) \subset(\mathrm{ES}) \subset(\mathrm{Rf}) .
\end{gathered}
$$

\section{3. Свойства отделимости для солнца. Глобальный минимизатор и луна}

3.1. Отделимость конусом для солнц. В нелинейной теории приближений часто оказываются важными геометрические свойства, более слабые, чем выпуклость. В соответствии с геометрической формой теоремы Хана-Банаха замкнутые выпуклые множества характеризуются тем, что любую точку вне такого множества можно строго отделить от него посредством (замкнутой) гиперплоскости (открытого полупространства).

Оказывается, что аналогичный результат верен для солнц (а также строгих (прото)солнц) при замене открытого полупространства на открытый опорный конус: точка, не принадлежащая солниу, строго отделяется от него посредством выпуклого открытого опорного конуса. Это утверждение содержится в теореме 3.1 (см., например, [61], [173; лемма 3] и [222; гл. 3 и комментарии к $\S 1$ гл. III]). Чтобы сформулировать этот результат, напомним, что множество

$$
\stackrel{\circ}{K}(y, x)=\bigcup_{r>0} \stackrel{\circ}{B}(-r y+(r+1) x,(r+1)\|x-y\|),
$$

состоящее из гомотетичных раздутий шара $\stackrel{\circ}{B}(x,\|x-y\|)$ относительно точки $y$, называется опорным конусом $\stackrel{\circ}{K}(y, x)$ к шару $B(x,\|x-y\|)$ в его граничной точке $y$ (см. [222], [173]). Мы также будем использовать следующее эквивалентное представление для $\stackrel{\circ}{K}(y, x)$. Для точки $s$ на единичной сфере $S$ через $\mathscr{P}_{s}$ обозначим множество всех функционалов из $S^{*}$, достигающих максимума на $S$ в точке $s$ (где $S^{*}$ - единичная сфера сопряженного пространства); пусть $\mathscr{E}_{s}-$ множество всех экстремальных точек выпуклого $w^{*}$-компактного множества $\mathscr{P}_{s} \subset S^{*}$. Далее, для различных точек $x, y \in X$ определим $p=$ $(y-x) /\|y-x\|$. Тогда [222; лемма 3.1]:

$$
\begin{aligned}
\stackrel{\circ}{K}(y, x) & =\left\{z \mid f(z)<f(y) \forall f \in \mathscr{P}_{p}\right\} \\
& =\left\{z \mid f(z)<f(y) \forall f \in \mathscr{E}_{p}\right\} \\
& =\{z \in X \mid[z, y] \cap \stackrel{\circ}{B}(x,\|x-y\|) \neq \varnothing\} .
\end{aligned}
$$

Отметим, что множество $X \backslash \stackrel{\circ}{K}(y, x)$ всегда является солнцем [173]. 
ТЕОРема 3.1 (характеризация “солнц" в терминах отделимости). Пусть $X$ - линейное нормированное пространство.

1. Множество $\varnothing \neq M \subset X$ является солнцем в $X$ тогда и только тогда, когда дляккаждой точки $x \notin M$ найдется точка $y \in P_{M} x$ такая, что $\stackrel{\circ}{K}(y, x) \cap$ $M=\varnothing$.

2. Множество $\varnothing \neq M \subset X$ является строгим протосолнцем (строгим солнием) в $X$ тогда и только тогда для каждой точки $x \notin M$ и для любого $y \in P_{M} x$ выполнено $\stackrel{\circ}{K}(y, x) \cap M=\varnothing$ (соответственно, для каждой точки $x \notin M$ множество $P_{M} x$ непусто и выполнено $\stackrel{K}{K}(y, x) \cap M=\varnothing$ для любого $\left.y \in P_{M} x\right)$.

3. Множество $\varnothing \neq M \subset X$ является $\alpha$-солнцем в $X$ тогда и только тогда, когда для каждой точки $x \notin M$ найдется точка $y \in S(x, \rho(x, M))$ такая, что $\stackrel{\circ}{K}(y, x) \cap M=\varnothing$.

Данный результат также верен в произвольных несимметрично нормированных пространствах [3].

Таким образом, многие результаты из линейной и выпуклой теории приближений переносятся без изменения на случай солнц и строгих (прото)солнц.

Аналогичный результат об отделимости $\gamma$-солнц получен Л. П. Власовым [223] в терминах отделимости при помощи клиноидов, представляющих собой обобщение понятия клина (конуса).

3.2. Солнца и критерий Колмогорова ближайшего элемента. Б. Брозовский [64] (см. также [89], [193], [228], [69]) установил связь между понятием строгого (прото)солнца и известным критерием Колмогорова ближайшего элемента.

Множество $M$ называется множеством Колмогорова, если из того, что $x \notin M, y_{0} \in P_{M} x$, следует неравенство

$$
\min _{f \in \mathscr{E}_{x-y_{0}}} f\left(y-y_{0}\right) \leqslant 0 \quad \forall y \in M
$$

где $\mathscr{E}_{x-y_{0}}-$ множество крайних точек (выпуклого) множества

$$
\mathscr{P}_{x-y_{0}}:=\left\{f \in S^{*} \mid f\left(x-y_{0}\right)=\left\|x-y_{0}\right\|\right\} .
$$

Иными словами, $M$ - множество Колмогорова, если для любого $y_{0} \in P_{M} x$

$$
\left\{y \in M \mid f\left(y-y_{0}\right)>0 \text { для каждого } f \in \mathscr{E}_{x-y_{0}}\right\}=\varnothing .
$$

Понятие множества Колмогорова было введено Б. Брозовским и Р. Вегманом [63], [70]. В теореме 3.3 мы увидим, что строгие протосолнца суть в точности множества Колмогорова.

Условие $(\mathrm{K})$ всегда является достаточным для того, чтобы $y_{0} \in P_{M} x$. Для множеств Колмогорова (строгих протосолнц) условие (K) также является необходимым для того, чтобы $y_{0} \in P_{M} x$ (в точности, как при характеризации наилучших приближений для выпуклых множеств).

Следующие два результата, являющиеся переформулировкой критерия Колмогорова ближайшего элемента, содержатся в работах Б. Брозовского [65], [64], В. Брекнера [58], Д. Амира и Ф. Дойча [26], Л. П. Власова [222; теорема 3.1] и монографии Д. Браесса [56; § II.2.А]. 
Теорема 3.2. Пусть $M$ - множество существования в линейном нормированном пространстве $X$. Следующие условия эквивалентны:

1) $M$ - строгое солнце;

2) если $x \in X$ u $y_{0} \in M, y_{0} \in P_{M} x$ тогда и только тогда, когда $y_{0}-$ ближайшая точка $\kappa x$ из отрезка $\left[y_{0}, y\right]$ для любого $y \in M$;

3) $M$ - множество Колмогорова.

Теорема 3.3. Для замкнутого подмножества $M$ линейного нормированного пространства $X$ следующие условия эквивалентны:

1) $M$ - строгое протосолние;

2) для любого $y \in M$ множество $P_{M}^{-1} y:=\left\{x \in X \mid y \in P_{M} x\right\}-$ конус с вершиной у (возможно, невыпуклый или сводящийся к одной точке $y$ );

3) $M$ - множество Колмогорова.

Таким образом, теорему 3.1 можно рассматривать как один из вариантов критерия Колмогорова (теоремы 3.2 и 3.3 ).

Рассмотрим применения критерия Колмогорова в конкретных задачах теории наилучшего приближения.

Множество обобщенно-рациональных функций. Пусть

$$
\mathscr{R}_{V, W}=\left\{\frac{p}{q} \mid p \in V, q \in W, q(t)>0, t \in Q\right\}
$$

- множество обобщенно-рациональных функций в действительном или комплексном $C(Q), Q$ - хаусдорфов компакт, $V, W$ - выпуклые подмножества в $C(Q)$. Имеет место следующий результат (Л. Чонг и Дж. А. Ватсон [83]).

Теорема 3.4. 1) Элемент $y_{0} \in \mathscr{R}_{V, W}$ является элементом наилучшего приближения для $x \in C(Q)$ тогда и только тогда, когда

$$
\max _{\{t:\|x-y\|=|(x-y)(t)|\}} \operatorname{Re} \overline{(x-y)(t)}\left(y-y_{0}\right)(t) \leqslant 0 \quad \text { для всех } \quad y \in \mathscr{R}_{V, W} .
$$

2) Элемент $y_{0} \in \mathscr{R}_{V, W}$ является единственным элементом наилучшего приближения для $x \in C(Q)$ тогда и только тогда, когда

$$
\max _{\{t:\|x-y\|=|(x-y)(t)|\}} \operatorname{Re} \overline{(x-y)(t)}\left(y-y_{0}\right)(t)<0 \quad \text { для всеx } \quad y \in \mathscr{R}_{V, W} \backslash\left\{y_{0}\right\} .
$$

Теперь из теоремы 3.3 следует, что $\mathscr{R}_{V, W}$ - строгое протосолнце в $C(Q)$.

Аналогичный результат в более узком случае при линейных $V, W$ был получен Брозовским и Вегманом [70], которые показали, что $\mathscr{R}_{V, W}-$ множество Колмогорова в $C(Q)$ (и, значит, строгое протосолнце).

С другими аппроксимативными свойствами $\mathscr{R}_{m, n}$ и $\mathscr{R}_{V, W}$ можно ознакомиться в монографиях Э. Чени [81], Д. Браесса [56], В.И. Бердышева и Л. В. Петрак [42], а также в работах Г.Ш. Рубинштейна, Д. Браесса [57], Ч. Данхема [91], [94], С. В. Конягина [140] и К. С. Рютина [185]-[187] и др.

Множество экспоненциальных сумм с неотрицательными коэффициентами

$$
E_{n}^{+}:=\left\{\sum_{j=1}^{n} \alpha_{j} e^{t_{j} x} \mid \alpha_{j} \geqslant 0, t_{j} \in \mathbb{R}\right\}
$$


является чебышёвским солнцем в $C[a, b]$. Строгая протосолнечность $E_{n}^{+}$вытекает из критерия Колмогорова с учетом того, что при приближении экспоненциальными суммами с неотрицательными коэффициентами наилучшие приближения характеризуются через альтернанс. Проксиминальность множества $E_{n}^{+}$в $C[a, b]$ установлена Э. Шмидтом [188; следствие 3]. Единственность наилучшего приближения вытекает из известной характеризации Данхема [92] множеств единственности в терминах тотальной регулярности (см. [57] применительно к $E_{n}^{+}$). Как и в случае приближения дробно-рациональными функциями, оператор наилучшего приближения на множество $E_{n}^{+}$имеет точки разрыва. Однако, как и в случае приближения дробями $\mathscr{R}_{m, n}$, из оператора почти наилучшего приближения на $E_{n}^{+}$существует непрерывная $\varepsilon$-выборка для любого $\varepsilon>0$.

Множество экспоненциалъных сумм

$$
E_{n}:=\left\{\sum_{j=1}^{n} \alpha_{j} e^{t_{j} x} \mid \alpha_{j}, t_{j} \in \mathbb{R}\right\}
$$

асимптотически выпукло в смысле Браесса [57] и Майнардуса-Шведта [164]. Напомним, что множество $M \subset C(Q)$ называется асимптотически выпуклым, если для любых $y, y_{0} \in M$ найдутся такие положительная функция $g \in C(Q)$ и непрерывное отображение $[0,1] \ni t \mapsto y_{t} \in M$, что

$$
\left\|(1-t g) y_{0}+t g y-y_{t}\right\|=o(t), \quad t \rightarrow 0 .
$$

В [57] показано, что асимптотически выпуклое множество $M \subset C(Q)$ является множеством Колмогорова и, следовательно, строгим протосолнцем. Как следствие, $E_{n}$ является строгим протосолнцем.

Представляют интерес также множества вида

$$
F_{n}:=\left\{\sum_{j=1}^{n} f_{j}\left(t_{j} x+c_{j}\right) \mid t_{j} x+c_{j} \in \Pi_{j}\right\},
$$

где $\left\{f_{j}: \mathbb{R} \rightarrow \mathbb{R}\right\}$ - набор монотонных функций, а $\left\{\Pi_{j}\right\}$ - набор промежутков (см. с. 56$)$. В пространстве $C[a, b]$ множество $F_{n}$ обладает непрерывной аддитивной $\varepsilon$-выборкой для любого $\varepsilon>0$. Частные случаи $F_{n}-$ это, например, $n$-звенные сплайны минимального дефекта с нефиксированными узлами и множество $E_{n}^{+}$. Если сплайны не имеют минимальный дефект, то утверждение о $\varepsilon$-выборке перестает быть верным (Лившиц [153]). Замыкание множества $F_{n}$ обладает непрерывной мультипликативной (аддитивной) $\varepsilon$-выборкой для любого $\varepsilon>0$.

В невырожденных случаях $E_{n}$ не является замкнутым множеством. К примеру, для $y_{m}(x):=m\left(e^{x / m}-1\right) \in E_{2}, m \in \mathbb{N}$, имеем $\left\|y_{m}(x)-x\right\| \rightarrow 0$. Соответственно, очень часто вместо множества $E_{n}$ рассматривается его замыкание $\bar{E}_{n}$ - множество расширенных экспоненциальных сумм, которое уже является множеством существования в $C[a, b]$ (см., например, [56; § IV.2.B]).

Множества $\bar{E}_{n}$ расширенных экспоненциальных сумм возникают как пространства решений линейных дифференциальных уравнений с постоянными 
коэффициентами, характеристические полиномы которых имеют лишь действительные корни:

$$
a_{n} y^{(n)}+\cdots+a_{1} y^{\prime}+a_{0} y=c\left(D-\lambda_{1}\right) \cdots\left(D-\lambda_{k}\right) y=0
$$

где

$$
\lambda_{1}, \ldots, \lambda_{k} \in \mathbb{R}, \quad k \leqslant n, \quad D:=\frac{d}{d x}, \quad a_{1}^{2}+\cdots+a_{n}^{2}=1, \quad y \in C^{n}[a, b] .
$$

Приближения экспоненциальными суммами возникают в широком классе прикладных задач (задачи теплопроводности, структурного анализа, радиоактивного распада и т. п.), где приближенные значения функции естественно выражаются в виде экспоненциальных сумм. При этом вектор параметров может быть записан как прямая сумма двух $n$-мерных векторов $a_{1}$ и $a_{2}$ :

$$
a=a_{1} \oplus a_{2}, \quad a_{1}=\left(\alpha_{1}, \ldots, \alpha_{n}\right), \quad a_{2}=\left(t_{1}, \ldots, t_{n}\right) .
$$

Приближение $\gamma$-полиномами. Пусть $Q \subset \mathbb{R}, I$ - интервал на прямой $\mathbb{R}$, $\gamma \in C(Q, I)$. Функция

$$
u(x)=\sum_{j=1}^{n} \alpha_{j} \gamma\left(t_{j}, x\right)
$$

называется $\gamma$-полиномом [56; гл. VII]; порядок полинома $u$ равен $n$, если $u$ не может быть представлен в виде суммы $n-1$ слагаемых. Экспоненциальные суммы являются частным случаем $\gamma$-полиномов с ядром $\gamma(t, x)=e^{t x}$. Часто также рассматривают ядра $\operatorname{ch} t x, x^{t}$ (полиномы Мюнца), $(1+x t)^{-1}, \operatorname{arctg} t x$, $(x-t)_{+}^{n}$ и $\log (1+t x)$. Если ядро $\gamma$ удовлетворяет условию расширенной знаковой регулярности (extended sign-regular) [56; с. 184], то множество $\gamma$-полиномов удовлетворяет локальному и глобальному условиям Хаара [57] и, как следствие, является строгим протосолнцем.

Приближение матрицами. Приближениями в пространстве матриц также занимались Х. Беренс, М. Финцель, Х. Ю. Шмидт, А.Р. Алимов и др. (см. [22]). В качестве аппроксимирующих множеств рассматривались различные классы матриц, например

$$
\mathscr{S}_{k}^{1}=\left\{S \in \mathbb{C}^{n \times n} \mid \sum_{1 \leqslant j_{1}<j_{2}<\cdots<j_{k} \leqslant n} \sigma_{j_{1}}(S) \sigma_{j_{2}}(S) \cdots \sigma_{j_{k}}(S) \leqslant 1\right\}
$$

где $\sigma_{1}(S), \sigma_{2}(S), \ldots, \sigma_{n}(S)$ - сингулярные числа матрицы $S$, и

$$
\mathscr{S}_{k}^{0}=\left\{S \in \mathbb{C}^{n \times n} \mid \operatorname{rank} S \leqslant k\right\} .
$$

С использованием условия Колмогорова в [22] было установлено, что в пространстве $C^{n \times n}$ со спектральной нормой $\mathscr{S}_{k}^{1}-$ чебышёвское солнце с глобально липшицевым оператором метрического проектирования, а $\mathscr{S}_{k}^{0}$ - солнце, обладающее липшицевой выборкой из оператора наилучшего приближения. 
3.3. Солнца и LG-множества. В этом пункте рассматривается задача о связи солнечности и глобальной минимизации.

Замкнутое множество $M \neq \varnothing$ называется LG-множеством или глобальным минимизатором (см., например, [70], [92]), если для любого $x \notin M$ каждый локальный минимум функции $\Phi_{x}(y)=\|y-x\|, y \in M$, является глобальным, что объясняет происхождение термина "LG-множество" (local-global). Иными словами, из того, что $y \in P_{M \cap V} x, V:=B(y, \varepsilon)$, следует, что $y \in P_{M} x$.

Из критерия Колмогорова также вытекает (см. [70; с. 382]), что любое строгое солнце (строгое протосолнце) $M$ является LG-множеством. Легко проверить, что обратное утверждение в общем случае неверно: дополнение к открытому шару не является солнцем, однако в строго выпуклом пространстве для этого множества любой локальный минимум функции $\Phi_{x}(y)=\|y-x\|$ является глобальным.

Рассмотрим множество $S_{m, k}$ сплайнов (в смысле Шумакера) степени $m \geqslant 1$ с $k \geqslant 1$ нефиксированными (кратными) узлами на отрезке $[a, b]$. (По поводу определения и некоторых свойств таких сплайнов мы отсылаем читателя к монографиям Г. Нюрнбергера [172], Л. Л. Шумакера [191].) Из критерия Колмогорова следует, что $S_{m, k}$ не является строгим солнцем в $C[a, b]$ (хорошо известно, что $S_{m, k}$ является множеством существования - Л. Шумакер, см., например, [172], - однако при приближении такими сплайнами множества глобальных и локальных наилучших приближений не совпадают (Г. Нюрнбергер [171], Д. Браесс [56]); при этом до сих пор не известен критерий, когда локальное наилучшее приближение в этом случае является глобальным). Также не известно, является ли $S_{m, k}$ солнцем. Множество $\widehat{S}_{m, k}$ сплайнов (минимальной гладкости) с нефиксированными простыми узлами также является множеством существования (см. [153; следствие 2.3]). Из недавних результатов о приближении множествами $\widehat{S}_{m, k}$ отметим работы Е. Д. Лившица [153]-[155], в которых рассматривались вопросы устойчивости оператора $\varepsilon$-проекции на $\widehat{S}_{m, k}$ в пространствах $C[0,1]$ и $L^{p}[0,1]$. Вопрос о солнечности $\widehat{S}_{m, k}$ остается открытым.

Для формулировки результатов о связи солнечности и задачи глобальной минимизации нам потребуется несколько определений. Пусть $\varnothing \neq M \subset X$. Точка $y_{0} \in M$ называется лунной точкой, если

$$
y_{0} \in \overline{M \cap \stackrel{\circ}{K}\left(y_{0}, x\right)} \quad \text { при условии, что } \quad x \in P_{M}^{-1}\left(y_{0}\right) \quad \text { и } \quad M \cap \stackrel{\circ}{K}\left(y_{0}, x\right) \neq \varnothing .
$$

Множество $M$ называется луной, ${ }^{2}$ если все его точки $y_{0}$ лунные.

Понятие луны было введено Амиром и Дойчем [26] и далее изучалось в [66], [225], [69], [68], [57], [56], [194]. Луна является обобщением протосолнца: известно (Амир и Дойч [26], Брозовский и Дойч [66]), что строгое протосолнце всегда является LG-множеством (глобальным минимизатором), a LG-множество, в свою очередь, является луной (см. теорему 3.5 ниже).

Брозовский и Дойч [66] назвали пространство (MS)-пространством, если в нем класс лун совпадает с классом строгих протосолнц (сокращение (MS)

\footnotetext{
${ }^{2}$ В [26] дается примечание, что изначально Брозовский и Дойч называли такие множества знаково регулярными (sign regular). Термин "луна" появился в понедельник ("Moonday") 21.09.1969, что объясняет название [26].
} 
происходит от английского "every $m$ oon in $X$ is a $s$ un"). Известно [26], что пространства $C(Q)\left(Q\right.$ - хаусдорфов компакт), $C_{0}(Q)(Q$ - локально компактное хаусдорфово пространство), $\ell^{1}(S)$ ( $S$ - произвольное множество), а также произвольное конечномерное полиэдральное пространство [69] являются (MS)-пространствами. Более подробно см. [25; § 2.3].

Отметим также, что никакое строго выпуклое пространство не является (MS)-пространством [26]: единичная сфера строго выпуклого пространства является луной, но не является строгим протосолнцем (и вообще солнцем в каком бы то ни было смысле). Соответственно, в строго выпуклых пространствах класс строгих солнц у́же класса лун. Пример луны, не являющейся LG-множеством (и, соответственно, строгим (прото)солнцем) дается подмножеством

$$
M=\left\{\begin{array}{l|l}
(x, y) \in \mathbb{R}^{2} & \frac{x^{2}}{4}+y^{2} \geqslant 1
\end{array}\right\}
$$

евклидовой плоскости (см. [56]).

В заключение раздела сформулируем теорему Брозовского-Вегмана [66], [69], [225] о связи лунности и строгой протосолнечности. Особенно примечательно то, что в (MS)-пространствах строгая протосолнечность характеризуется ORL-непрерывностью метрической проекции. Напомним, что $P_{M}$ называется ORL-непрерывной (outer radially lower continuous) [66] в точке $x$, если из условий

$$
y \in P_{M} x, \quad\left(x_{n}\right) \subset\{y+\lambda(x-y) \mid \lambda \geqslant 1\}, \quad x_{n} \rightarrow x
$$

следует, что

$$
\rho\left(y, P_{M} x_{n}\right) \rightarrow 0
$$

(см. далее раздел 5).

ТеОРема 3.5. Пусть $M$ - подмножество линейного нормированного пространства $X$. Тогда из (i) следует $(i+1), i=1,2,3$ :

(1) $M$ - строгое протосолнце;

(2) метрическая проекиия $P_{M}$ является ORL-непрерывной во всех точках;

(3) $M$ - LG-множество (глобальный минимизатор);

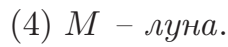

Если X является (MS)-пространством, то условия (1)-(4) эквивалентнъ.

3.4. Характеризации солнц в различных пространствах. Власов [224] получил характеризацию пространств, в которых всякое двухточечное множество является $\alpha$-солнцем ( $\gamma$-солнцем). В частности [224], если в банаховом пространстве $X$ всякое двухточечное подмножество является $\alpha$-солнцем ( $\gamma$-солнцем), то $X$ не сепарабельно (соответственно не является асплундовским пространством). Также в [224] даются необходимые и достаточные условия того, что заданное двухточечное множество является $\alpha$-солнцем ( $\gamma$-солнцем). $\beta$-солнца охарактеризованы Власовым в терминах, сходных с почти выпуклостью [221]. Иными словами, замкнутое множество $M$ является $\beta$-солнцем в том и только том случае, когда любой открытый шар, не пересекающийся с $M$, можно поместить в открытый шар сколь угодно большого радиуса, не пересекающийся с $M$. Замкнутое множество $M$ является $\gamma$-солнцем в том и только том 
случае, когда любой замкнутый шар, находящийся на положительном расстоянии от $M$, можно поместить в замкнутый шар сколь угодно большого радиуса, находящийся на положительном расстоянии от $M$. В связи с этим появляется новое понятие солнечности - метакси-солнечность ${ }^{3}$ : замкнутое множество $M$ является метакси-солнием, если любой замкнутый шар, не пересекающийся с $M$, можно поместить в замкнутый шар сколь угодно большого радиуса, не пересекающийся с $M$. Метакси-солнечность является промежуточным понятием между $\beta$ - и $\gamma$-солнечностью. Существует метакси-солнце, не являющееся ни $\beta$-, ни $\gamma$-солнцем.

В пространстве $\ell^{\infty}(n)$ геометрическая характеризация солнц получена Беренсом и Хетцельтом [44], а строгих солнц и чебышёвских множеств - Алимовым (соответственно [9] и [12]).

Строгие протосолнца в пространстве $C(Q)$ были охарактеризованы В. Янгом, Ч. Ли и Дж. А. Ватсоном [228] в терминах критерия Колмогорова, критерия Папини и свойства слабой промежуточности. В $C(Q, X)$ и $C_{0}(Q, X)$ строгие протосолнца были охарактеризованы П. Шварцем [189].

Понятие регулярного подмножества линейного нормированного пространства было введено Брозовским [63; §1] (см. также [57]) и независимо Данхемом [92] в случае $C(Q)$, а для общих пространств - в [70] (см. также [194]). Сначала дадим определение в случае приближения в равномерной норме.

Замкнутое множество $M \subset C(Q)$ называется регулярным (по Брозовскому-Вегману), если для любых $x, y \in M$ и любого замкнутого множества $A \subset Q$ такого, что $\inf _{t \in A}|x(t)-y(t)|>0$, найдется последовательность $\left(v_{n}\right)$ точек из $M$ такая, что

$$
v_{n} \rightarrow y \quad \text { и } \quad\left(v_{n}(t)-y(t)\right)(x(t)-y(t))>0 \quad \text { для всех } t \in A .
$$

Геометрически условие регулярности в $C(Q)$ достаточно прозрачно. Пусть $M$ - регулярное замкнутое подмножество пространства $C(Q)$ и $x, y \in M$. Тогда $x$ и $y$ можно соединить кривой $k(\tau) \subset M$, координатная проекция которой содержится в относительной внутренности "прямоугольника" $[[x, y]:=\{z \mid z(t) \in$ $[x(t), y(t)]\}$, при этом, если $x\left(t_{0}\right) \neq y\left(t_{0}\right)$ в некоторой точке $t_{0}$, то координатная кривая $k(\tau)\left(t_{0}\right)$ строго монотонна по $\tau$.

$\mathrm{K}$ примеру, если $M$ - регулярное подмножество пространства $\ell^{\infty}(n)$ и $x, y$ принадлежат $M$, причем все координаты $x$ и $y$ различны, то $x$ и $y$ можно соединить строго монотонной кривой $k(\tau)$, содержащейся в $M$; последнее означает, что координатные функции $k_{i}(\tau)$ строго монотонны по $\tau$ для любого $i=1, \ldots, n$.

Непустое подмножество $M$ произвольного линейного нормированного пространства $X$ называется регулярным (по Брозовскому-Вегману) в точке $v_{0} \in M$ (см. [70; §4], [194; §5.3]), если для любых $x \notin M, v \in M, \lambda>0$ и любого $w^{*}$-замкнутого $A \subset \operatorname{ext} S^{*}$, содержащего $\mathscr{E}_{x-v_{0}}$ и такого, что $f\left(v-v_{0}\right)>0$, $f \in A$, найдется элемент $v_{\lambda} \in M$ со следующими свойствами:

1) $f\left(v_{\lambda}-v_{0}\right)>f\left(x-v_{0}\right)-\left\|x-v_{0}\right\|$ при всех $f \in A$;

2) $\left\|v_{\lambda}-v_{0}\right\|<\lambda$.

\footnotetext{
${ }^{3}$ По-гречески $\mu \epsilon \tau \alpha \xi \dot{v}$ означает "между".
} 
Замкнутое множество $M$ называется регулярным, если оно регулярно в каждой своей точке. Здесь, как и выше, для $x \in X$

$$
\Sigma_{x}:=\left\{f \in S^{*} \mid f(x)=\|x\|\right\},
$$

$\mathscr{E}_{x}-$ множество крайних точек $\Sigma_{x}$, ext $S^{*}$ - множество крайних точек единичной сферы $S^{*}$ сопряженного пространства $X^{*}$. Следующий результат установлен Брозовским и Вегманом [70; лемма 1].

ПредложЕНИЕ 3.1 (вариационная лемма). Пусть $v_{0}$ - регулярная точка множества $M, x \in X, u$ пусть $v \in M-$ такая точка, что $f\left(v-v_{0}\right)>0$ при всех $f \in \mathscr{E}_{x-v_{0}}$. Тогда для каждого $\lambda>0$ множество

$$
U\left(\lambda, v_{0}\right):=\left\{y \in M \mid\left\|y-v_{0}\right\|<\lambda\right\}
$$

содержит точку ул такую, что

$$
\left\|x-y_{\lambda}\right\|<\left\|x-v_{0}\right\|
$$

Из вариационной леммы следует, что множество Колмогорова (строгое протосолнце) является LG-множеством (теорема 3.5).

Следующий результат содержится в работах Брозовского и Вегмана [70; предложение 8], Власова [222].

Теорема 3.6. Пусть $\varnothing \neq M \subset X$. Тогда следующие условия эквивалент$H b l$ :

а) $M$ является строгим протосолнщем;

b) $\stackrel{\circ}{K}(y, x) \cap M=\varnothing$ для любых $x \notin M, y \in P_{M} x$;

c) $М$ регулярно;

d) $M$ удовлетворяет критерию Колмогорова: $y_{0} \in P_{M} x$ тогда и только тогда, когда для любого у $\in M$ найдется функционал $f \in S^{*}$ такой, что

$$
f\left(x-y_{0}\right)=\left\|x-y_{0}\right\| \quad u \quad f\left(y-y_{0}\right) \leqslant 0 .
$$

Еще одно условие типа регулярности ( $H$-регулярность), эквивалентное условиям теоремы 3.6, было предложено М. Зоммером [196] (см. также [225]).

\section{4. Классы солнц, соотношения между ними и выпуклость. Точки светимости}

Из геометрической формы теоремы Хана-Банаха легко вытекает, что всякое выпуклое множество является строгим протосолнцем, а всякое выпуклое множество существования - строгим солнцем (см., например, [194; с. 83], [222; следствие 3.1]). Кли [134] и независимо Ефимов и Стечкин [96] (см. также [217], [26], [193; с. 344]) установили частично обратный результат, именно: в гладком линейном нормированном пространстве всякое солнце выпукло (этот результат доказывался ими для строгих солнц; перенесение на случай солнц тривиально). Отметим следующую характеризацию гладких пространств: линейное нормированное пространство является гладким тогда и только тогда, 
когда всякое солнце (строгое солнце) выпукло (см., например, [56; теорема 2.5]). Для строгих протосолнц ответ оказывается иным.

Непустое замкнутое подмножество $M$ называется почти выпуклым, если для любого замкнутого шара $B(x, r)$, находящегося на положительном расстоянии от $M$, найдется замкнутый шар $B(y, R) \supset B(x, r)$ сколь угодно большого радиуса $R$, также находящийся на положительном расстоянии от $M$. Данное понятие введено Власовым [219]. Отметим, что в зарубежной литературе почти выпуклые множества также называют аппроксимативно выпуклыми (см., например, [55]), что приводит к некоторой путанице, поскольку под последними иногда [217], [219] понимаются P-выпуклые множества (множества, метрическая проекция на которые выпуклозначна), а иногда - множества $M$ со свойством $\rho((x+y) / 2, M) \leqslant 1$ для любых $x, y \in M$.

В теореме 4.1, восходящей к Ефимову, Стечкину и Кли, рассматриваются условия на пространство, при которых всякое солнце выпукло. В настоящем виде теорема 4.1 сформулирована Власовым [222]. Теорема 4.2 содержится в [222] (и следует из критерия Колмогорова с учетом того, что в гладком пространстве опорный конус $\stackrel{K}{K}(x, y)$ является открытым полупространством).

Теорема 4.1. Следующие условия на банахово пространство X эквивалентны:

a) $X$ гладко;

b) каждое $\alpha$-солнце в $X$ выпукло;

c) каждое солние в $X$ выпукло;

d) каждое строгое солние в $X$ выпукло;

е) каждое $P$-выпуклое строгое солние в $X$ выпукло.

Теорема 4.2. Следующие условия на банахово пространство $X$ эквивалентны:

a) $X^{*}$ строго выпукло;

b) каждое $\delta$-солние ( $\gamma$-солнце, почти выпуклое множество) в $X$ выпукло;

c) класс $\delta$-солни, ( $\gamma$-солни, почти выпуклых множеств) в X совпадает с классом непустых выпуклых замкнутых подмножеств $X$.

В связи с теоремой 4.2 отметим (см. [201]), что класс банаховых пространств, у которых сопряженное пространство строго выпукло, у́же класса гладких банаховых пространств. При этом понятно, что если $X^{*} \in(\mathrm{R})$, то $X \in(\mathrm{S})$.

Как следствие, в гладком рефлексивном пространстве класс $\delta$-солнц $(\gamma$-солнц, почти выпуклых множеств) совпадает с классом непустых выпуклых замкнутых множеств.

Для строгих протосолнц ответ на вопрос об их выпуклости отличен от ответа в случае строгих солнц. При этом оказывается, что в общей ситуации строгое протосолнце (если только оно не есть строгое солнце) не является выпуклым и даже почти выпуклым множеством! Рассмотрим соответствующие простые примеры.

Пример 4.1. Если $X$ нерефлексивно, то в $X$ содержится невыпуклое строгое протосолнце (дополнение к антипроксиминальной гиперполосе). 
Действительно, в соответствии с классической теоремой Джеймса, если $X$ нерефлексивно, то существует функционал $f \in S^{*}$, не достигающий нормы на $S$. Гиперплоскость, порождаемая таким функционалом, замкнута и антипроксиминальна. Взяв две параллельные гиперплоскости, порождаемые таким функционалом, и взяв дополнение к открытой гиперполосе между этими гиперплоскостями, мы получаем замкнутое невыпуклое антипроксиминальное строгое протосолнце, не являющееся строгим солнцем.

Можно также построить пример антипроксиминального строгого протосолнца в виде "клина". Пусть $f, g, f \neq \lambda g(\lambda \in \mathbb{R})$, - два функционала, не достигающие своей нормы (легко видеть, что таких функционалов для $X \notin(\mathrm{Rf})$ существует по крайней мере два). Вообще, если $X$ нерефлексивно, то множество функционалов из $X^{*}$, не достигающих своей нормы, достаточно велико. Отметим следующий результат (М. Хименес-Севилла, Х. Морено [128]). Пусть $X-$ сепарабельное банахово пространство. Тогда или $S^{*} \backslash N A$ является $w$-плотным в $S^{*}$ или $X \in(\mathrm{Rf})$. Здесь $N A$ - множество функционалов из $S^{*}$, достигающих своей нормы. Ясно, что замкнутое (антипроксиминальное) множество $M=X \backslash\{x \mid f(x)<1, g(x)<1\}$ является невыпуклым строгим протосолнцем (это следует, например, из теоремы 3.3).

ПримеР 4.2. Построим пример невыпуклого строгого протосолнца (замкнутой выпуклой каверны) в некотором гладком сепарабельном банаховом пространстве с дифференцируемой по Фреше нормой. В этом примере, как и выше, искомое протосолнце $M$ антипроксиминально. В. С. Балаганский [29] построил пример строго выпуклого банахова пространства $X$ (соответствующим образом перенормированное подпространство пространства $c_{0}$ конечной коразмерности) с дифференцируемой по Фреше нормой (такое $X$ - заведомо гладкое) и в нем - замкнутое антипроксиминальное выпуклое ограниченное тело $C$. Воспользуемся еще одним утверждением Балаганского [31; лемма 1]: если $K \subset X$ - выпуклое замкнутое антипроксиминальное тело в банаховом пространстве $E$, то множество $\overline{E \backslash K}$ тоже антипроксиминально. Как следствие, замкнутое множество $M:=\overline{X \backslash C}$ антипроксиминально и является невыпуклым строгим протосолнцем в гладком пространстве $X$. Ясно, что такое $M$ не является почти выпуклым.

Отметим, что пространство из примера 4.2 имеет дифференцируемую по Фреше норму, но не является рефлексивным, поскольку в рефлексивном пространстве выпуклое замкнутое множество является множеством существования.

Остановимся вкратце на вопросе о том, в каких пространствах класс строгих солнц совпадает с классом строгих протосолнц.

Имеют место следующие результаты о совпадении классов солнц. В теореме 4.3 первый результат установлен в [221], [222; теорема 3.6], по поводу второго см. [32; предложение 2.2].

Tеорема 4.3. Пусть $X \in(\mathrm{LUR})$, и пусть $M \subset X$ является $\beta$-солнием или $\gamma$-солнцем существования. Тогда $M$ - чебышёвское солние.

Протосолнце, для которого множество точек существования всюду плотно в пространстве, является почти выпуклым; следовательно, в пространствах 
Ефимова-Стечкина (и, в частности, в равномерно выпуклом пространстве) всякое протосолнце почти выпукло. В этой связи имеет место следующий результат.

Tеорема 4.4. В банаховом пространстве $X \in(\mathrm{UR})$ класс строгих солни, совпадает с классом строгих протосолни, а такэсе с классом чебышёвских солни.

ДокАЗАтельство. Известно [142], что в пространствах Ефимова-Стечкина и, в частности, в равномерно выпуклых пространствах для замкнутого множества $M$ множество точек его аппроксимативной компактности является множеством второй категории (т. е. содержит плотное $G_{\delta}$-множество). Соответственно, если шар $V:=B(x, r)$ находится на положительном расстоянии от $M$, то сколь угодно близко к $x$ можно найти точку аппроксимативной компактности $x^{\prime}$ такую, что шар $V^{\prime}:=B\left(x^{\prime}, r^{\prime}\right) \supset V$ не пересекается с $M$ при некотором $r^{\prime}>0$. Для $x^{\prime}$ множество ближайших $P_{M} x^{\prime}$ непусто и, вследствие протосолнечности $M, x$ - точка строгой солнечности. Поскольку $\stackrel{\circ}{K}\left(y, x^{\prime}\right) \cap M=\varnothing$ для любого $y \in P_{M} x^{\prime}$, то из представления (3.1) следует, что шар $V^{\prime}$ содержится в замкнутом шаре сколь угодно большого радиуса, находящемся на положительном расстоянии от $M$. Таким образом, $M$ почти выпукло (и, значит, является $\gamma$ - и $\delta$-солнцем). Теперь из [32; предложение 1.12$]$ вытекает, что $M$ аппроксимативно компактно и, следовательно, проксиминально. Окончательно, $M$ - чебышёвское солнце по теореме 4.3. Теорема доказана.

ПровлемА 1. Охарактеризовать банаховы пространства, в которых всякое строгое протосолнце является строгим солнцем.

С проблемой выпуклости солнц связана следующая задача: охарактеризовать (конечномерные) пространства (размерности $\geqslant 3$ ), в которых всякое ограниченное строгое солнце (солнце, связное $\alpha$-солнце) выпукло. В двумерном случае задача о выпуклости чебышёвских множеств совпадает с задачей о выпуклости ограниченных чебышёвских множеств: всякое чебышёвское множество на нормированной плоскости $X$ выпукло тогда и только тогда, когда $X$ гладко (Манн [158], Бунт [78], Моцкин [168]). В случае $\operatorname{dim} X \geqslant 3$ это уже не так (Бердышев [37], Брондстед [61]). В этой связи напомним, что Царьков [202] (см. также [205], [204], [72]) установил, в частности, что в конечномерном линейном нормированном пространстве $X$ всякое ограниченное чебышёвское множество (ограниченное $P$-ацикличное множество, ограниченное $B$-ацикличное множество) выпукло тогда и только тогда, когда множество экстремальных точек единичной сферы $S^{*}$ сопряженного пространства плотно в ней. Такое пространство не обязано быть гладким в размерности $\geqslant 3$. Примеры таких пространств приведены в [202] и [72]. По поводу бесконечномерного случая см. [204], [205]. Также отметим, что Царьков [207; теорема 2.4] показал, что в конечномерном банаховом пространстве множество экстремальных точек сопряженной сферы $S^{*} \subset X^{*}$ плотно в ней тогда и только тогда, когда каждое ограниченное множество существования с полунепрерывной снизу метрической проекцией выпукло. 
По-видимому, для ограниченных строгих солнц (и даже солнц) ответ будет аналогичен приведенной выше характеризации для ограниченных чебышёвских множеств. Этот вопрос имеет давнюю историю, и до сих пор полного ответа на него нет.

Провлема 2. Охарактеризовать (конечномерные) пространства (размерности $\geqslant 4$ ), в которых всякое ограниченное строгое солнце (солнце, связное $\alpha$-солнце) выпукло.

В связи с проблемой 2 еще раз отметим, что существуют примеры негладких конечномерных пространств (размерности $\geqslant 3$ ), в которых всякое ограниченное чебышёвское множество (ограниченное чебышёвское солнце) выпукло, но существует неограниченное невыпуклое чебышёвское множество. Положительный ответ в проблеме 2 для строгих солнц в размерности 3 был недавно получен первым автором.

Отметим еще один результат о совпадении классов "солнц".

Теорема 4.5. Рассмотрим следующие условия на множество $M$ линейного нормированного пространства $X$ :

а) $M$ является $\delta$-солнием;

b) $М$ является $\gamma$-солнием;

c) $M$ почти выпукло.

Тогда $\mathrm{b}) \Leftrightarrow \mathrm{c})$, а если $Х$ банахово, то все три условия эквивалентны.

Эквивалентность b) $\Leftrightarrow$ c) в теореме 4.5 установлена Власовым [221], [222; теорема 3.3], им же [222; следствие 3.2] построен пример $\delta$-солнца, не являющегося $\gamma$-солнцем (естественно, в неполном линейном нормированном пространстве). Следуя рассуждениям Власова, докажем а) $\Leftrightarrow \mathrm{b})$. Пусть $M$ является $\delta$-солнцем. Возьмем произвольные $x \notin M, r>0, \sigma>1$. Введем порядок в шаре $V:=B(x, r)$ следующим образом: положим $z \preceq z^{\prime}$, если $\left\|z-z^{\prime}\right\| \leqslant \sigma\left(\rho\left(z^{\prime}, M\right)-\rho(z, M)\right)$. Отношение $\preceq$ является отношением порядка и, в силу полноты пространства, удовлетворяет условиям леммы Цорна о максимальном элементе (см. $[222 ; \S 3.2])$. Поэтому для $x$ существует максимальный элемент $v \in V, v \succeq x$. Предположим, что $v \in \operatorname{int} V$. Поскольку $\rho(v, M) \geqslant \rho(x, M)>0$, то $v \notin M$. По определению $\delta$-солнца найдутся $z_{n} \neq v$, $z_{n} \rightarrow v$, такие, что

$$
\frac{\rho\left(z_{n}, M\right)-\rho(v, M)}{\left\|z_{n}-v\right\|} \rightarrow 1, \quad n \rightarrow \infty .
$$

Следовательно, поскольку $\sigma>1, v \in \operatorname{int} V$, то при некотором $n_{0}$ будем иметь $z_{n} \in V,\left\|z_{n}-v\right\| \leqslant \sigma\left(\rho\left(z_{n}, M\right)-\rho(v, M)\right)$ для $n \geqslant n_{0}$, что противоречит максимальности $v$. Итак, $v$ лежит на границе $V$. Из условия $x \preceq v$ следует, что $\|v-x\| \leqslant \sigma(\rho(v, M)-\rho(x, M))$. Положим $v_{\sigma}=v$. В силу равномерной непрерывности функции расстояния имеем

$$
\frac{1}{\sigma} \leqslant \frac{\rho\left(v_{\sigma}, M\right)-\rho(x, M)}{\left\|v_{\sigma}-x\right\|} \leqslant 1,
$$

т. e.

$$
\frac{\rho\left(v_{\sigma}, M\right)-\rho(x, M)}{\left\|v_{\sigma}-x\right\|} \rightarrow 1, \quad \sigma \rightarrow 1+0 .
$$


Поскольку $\left\|v_{\sigma}-x\right\|=r$, это показывает, что $M$ является $\gamma$-солнцем, что и требуется.

Другие результаты о соотношениях между классами солнц можно найти в работах Власова [222], [221], Кощеева [144] и обзорной работе Балаганского и Власова [32].

Отметим следующий достаточно простой, но важный результат. Если $M-$ строгое протосолнце, то

$$
\operatorname{conv} P_{M} x \subset S(x, \rho(x, M))
$$

(см., например, [70; предложение 13], а также [194; формула (5.32)]; отметим, что для солнц включение (4.1) уже перестает быть верным даже в плоском случае). В дополнение к данному утверждению отметим, что (4.1) также выполняется, если $M$ - множество существования, а оператор метрического проектирования $P_{M}$ полунепрерывен снизу в точке $x$ (см. [178; с. 56]).

Приведем важное свойство солнц (легко следующее из теоремы 3.1 и представления (3.2) для опорного конуса): если $y_{0}$ - точка светимости множества $M$ для точки $x \in X \backslash M$ и если $y \in M$, то

$$
\left[y_{0}, y\right] \cap \stackrel{\circ}{B}(x, \rho(x, M))=\varnothing ;
$$

если при этом $y \in P_{M} x$, то

$$
\left[y_{0}, y\right] \subset S(x, \rho(x, M))
$$

(при этом вовсе не обязательно, что $\left[y, y_{0}\right] \subset M$ даже для строгого солнца $M$ ).

ЗАмЕчАниЕ 4.1. В связи с (4.2) отметим, что в пространствах размерности $\geqslant 3$ строгое солнце $M$ не обязано быть $P$-звездным (множество $M$ называется звездным относительно $x$, если $[x, y] \subset M$ для любого $y \in M)$. Пример строгого солнца, не являющегося $P$-звездным, в $\ell^{\infty}(3)$ дается множеством

$$
\left\{x=\left(x^{(1)}, x^{(2)}, x^{(3)}\right) \mid x^{(2)}=\frac{1}{x^{(1)}}, 1 \leqslant x^{(1)} \leqslant 2,0 \leqslant x^{(3)} \leqslant 1\right\}
$$

(для точек "сверху" и "снизу" множества шар касается множества по куску гиперболы). По-видимому, можно надеяться, что в конечномерных $X$ солнца $P$-стягиваемы и $P$-ацикличны (в конечномерном случае эти два условия эквивалентны в классе монотонно линейно связных множеств; определение монотонной линейной связности приведено в разделе 9); частичный ответ на этот вопрос дается в разделе 9. Ответ на вопрос о $P$-звездности солнц положителен в двумерных $X$ (см. [43], [16]): для солнца $M$ в двумерном пространстве $P_{M} x$ является точкой, отрезком или объединением двух отрезков, имеющих одну общую концевую точку.

В заключение этого раздела остановимся вкратце на вопросе о светимости границы солнца. Иными словами, нас интересует вопрос, все ли граничные точки солнц являются точками светимости (т. е. удовлетворяют условию светимости (2.1) при некоторых $x)$. 
Для граничной точки $y$ подмножества $M$ пространства $X$ определим метрический прообраз точки $y$ :

$$
P_{M}^{-1}(y)=\left\{x \in X \backslash M \mid y \in P_{M} x\right\}
$$

Имеет место следующий результат [5].

ПРЕДЛОЖЕНИЕ 4.1. В конечномерном пространстве $X$ каждая граничная точка солниа является его точкой светимости.

В бесконечномерном случае это уже не так. В качестве примера рассмотрим гильбертов кирпич $M:=\left\{x \in \ell^{2}|| x^{(n)} \mid \leqslant 1 / n, n \in \mathbb{N}\right\}$ в пространстве $\ell^{2}$. Так как множество $M$ выпукло и компактно, то оно является строгим солнцем. Известно (см., например, [179; пример 1.9.1]), что граничную точку $0 \in \mathrm{bd} M$ нельзя (нестрого) отделить от $M$ гиперплоскостью. Из этого следует, что точка 0 не является точкой светимости множества $M$. Действительно, если бы точка 0 принадлежала $P_{M} x$ для какого-то $x \notin M$, то тогда по теореме 3.1 опорный конус $\stackrel{\leftrightarrow}{K}(0, x)$ не пересекался бы с $M$. Пространство $\ell^{2}$ гладкое, поэтому опорный конус - открытое полупространство, граница которого (нестрого) отделяет точку 0 от $M$. Противоречие с предыдущим утверждением об отделимости.

В связи с предложением 4.1 отметим, что для произвольного собственного замкнутого подмножества существования $M$ произвольного метрического пространства имеет место достаточно очевидное равенство

$$
\operatorname{cl}\left\{y \in \operatorname{bd} M \mid P_{M}^{-1}(y) \neq \varnothing\right\}=\operatorname{bd} M
$$

т. е. множество его граничных точек с непустым метрическим прообразом всюду плотно на границе $\operatorname{bd} M$ множества $M$.

\section{5. Классы связности. Связность чебышёвских множеств и множеств единственности}

В этом разделе изучаются структурные свойства связности в различных смыслах для чебышёвских множеств и их обобщений - множеств единственности. Вопрос о связности солнц будет рассмотрен в разделе 8.

Рассмотрим следующие классы множеств $M \subset X$ :

$(\mathscr{F})$ - класс непустых замкнутых множеств;

$(\mathscr{E})$ - класс множеств существования;

$(\stackrel{\circ}{B})$ - класс $\stackrel{\circ}{B}$-связных множеств;

$(B)$ - класс $B$-связных множеств;

$(P)$ - класс $P$-связных множеств $\left(P_{M} x\right.$ непусто и связно для любого $M \in(P))$;

$\left(P_{0}\right)$ - класс $P_{0}$-связных множеств $\left(P_{M} x\right.$ связно для любого $\left.M \in\left(P_{0}\right)\right)$.

Имеет место следующий результат, установленный независимо Невесенко [169] и Кощеевым [145]. 
ТеОРема 5.1. В линейном нормированном пространстве $X$ Р-связное множество является В-связным, если выполняется хотя бы одно из следующих условий:

а) $P_{M}$ полунепрерьвна сверху;

b) $P_{M}$ полунепрерывна снизу.

Отображение $F: X \rightarrow 2^{Y}$ называется полунепрерывным снизу в точке $x_{0}$, если для любой окрестности $O(y)$ любой точки $y \in F\left(x_{0}\right)$ найдется окрестность $O\left(x_{0}\right)$ такая, что $F(x) \cap O(y) \neq \varnothing \forall x \in O\left(x_{0}\right)$. Как обычно, $F$ полунепрерывно снизу, если оно полунепрерывно снизу в любой точке $x_{0} \in X$.

Напомним следующий факт. Пусть $X, Y$-банаховы пространства, $F: X \rightarrow 2^{Y}$. Тогда следующие утверждения эквивалентны [80; предложение 9.84]:

1) $F$ полунепрерывно снизу;

2) прообраз $F^{-1}(U):=\{x \in X \mid F(x) \cap U \neq \varnothing\}$ любого открытого множества $U \subset Y$ открыт;

3) для любых $x_{0} \in X, x_{n} \rightarrow x_{0}$ и любого $y \in F\left(x_{0}\right)$ найдется $y_{n} \in F\left(x_{n}\right)$, $n \in \mathbb{N}$, такая, что $y_{n} \rightarrow y$ при $n \rightarrow \infty$.

Невесенко [170] также получил ряд других условий, гарантирующих $B$-связность $P$-связного множества. Теорема 5.1 обобщает результат Власова [222; теорема 4.1], согласно которому в линейном нормированном пространстве $P$-связное множество с полунепрерывной сверху метрической проекцией $\stackrel{B}{B}$-связно.

Отметим также следующий результат (Царьков [203]), в котором условие на множество в теореме 5.1 ослабляется за счет ограничения на пространство.

Теорема 5.2. В пространстве Ефимова-Стечкина замкнутое $P_{0}$-связное множество $M$ является $\stackrel{\circ}{B}$-связным, т.е. $\left(P_{0}\right) \subset(\stackrel{\circ}{B})$.

ЗАмечАние 5.1. Теорема 5.2 восходит к одному результату Власова ([222; теорема 4.2], [220]), который рассматривал равномерно выпуклые пространства и $P$-связные множества (заведомо являющиеся множествами существования) без упоминания о множествах единственности. Однако при ее доказательстве Власовым не подразумевалось свойство существования элемента наилучшего приближения. Царьков распространил теорему 5.2 на $P_{0}$-связные множества и пространства Ефимова-Стечкина (см. также теорему 5.9 ниже). На несимметрично нормированные метризуемые равномерно выпуклые пространства теорема 5.2 частично обобщена П. А. Бородиным [52]: в равномерно выпуклом несимметрично нормированном пространстве $P$-связное множество $B$-связно. Е. Н. Сосов [197] перенес теорему 5.1 на равномерно выпуклые геодезические пространства.

В общем случае теорема 5.2 не имеет места: в произвольном банаховом пространстве чебышёвское множество не обязано быть связным - известный пример Данхема несвязного чебышёвского множества в $C[0,1]$ показывает, что

$$
(P) \neq(B) \cap(\mathscr{E})
$$

Множество $M$ называется $\varepsilon$-компактным [222], если для любого $x \notin M$ найдется $\varepsilon>0$ такое, что $P_{\varepsilon, M} x$ компактно; здесь $P_{\varepsilon, M}:=M \cap B(x, \rho(x, M)+\varepsilon)$ 
(аддитивная $\varepsilon$-проекция, или множество почти наилучших приближений). Отметим, что любое $\varepsilon$-компактное множество всегда аппроксимативно компактно, а ограниченно компактное - всегда $\varepsilon$-компактно.

Теорема 5.3. В пространстве Ефимова-Стечкина локально компактное, Р-компактное и Р-связное множество $\varepsilon$-компактно, аппроксимативно компактно, В-связно и обладает полунепрерывной сверху метрической проекиией.

В частном случае равномерно выпуклых пространств теорема 5.3 восходит к Власову [222; теорема 4.3]. Теорема 5.3 вытекает из того, что если $X \in(\mathrm{ES})$, то $\left(P_{0}\right) \cap(\mathscr{F}) \subset(\stackrel{\circ}{B})$ (теорема 5.2). Далее, известно, что всякое локально компактное, $P$-компактное и $\stackrel{B}{B}$-связное множество $M$ аппроксимативно компактно, $\varepsilon$-компактно, а его метрическая проекция полунепрерывна сверху (Власов [222; теорема 2.2]). Окончательно, $B$-связность обеспечивается теоремой 5.4.

Легко установить, что (см. [222; предложение 0.5])

$$
(B) \subset(\stackrel{\circ}{B}) \text {. }
$$

Частичный ответ на обратный вопрос дается следующей теоремой (Кощеев [143; теорема 8]), см. также теорему 5.1.

ТЕОРемА 5.4. В линейном нормированном пространстве аппроксиматив-

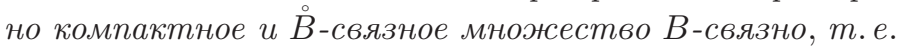

$$
(\mathrm{AC}) \cap(\stackrel{\circ}{B}) \subset(B) .
$$

Отметим следующие факты (Царьков [203]). В каждом бесконечномерном банаховом пространстве существует $P_{0}$-связное, но не $P$-связное замкнутое множество; в каждом сепарабельном бесконечномерном банаховом пространстве существует $\stackrel{\circ}{B}$-связное, но не $B$-связное замкнутое множество.

ПроблемА 3. Верно ли, что в каждом (несепарабельном) бесконечномерном пространстве существует $\stackrel{\circ}{B}$-связное, но не $B$-связное замкнутое множество?

ПроблемА 4. Верно ли, что в произвольном пространстве выполнено включение $(P) \cap(\stackrel{\circ}{B}) \subset(B)$ ?

Рассмотрим класс множеств

$$
\left(\stackrel{\circ}{B}_{l}\right)=\{M \subset X \mid \forall x \in X, \forall r \geqslant 0 \stackrel{\circ}{B}(x, r) \cap M \text { линейно связно }\} .
$$

Ясно, что если $M \in\left(\stackrel{\circ}{B}_{l}\right)$, то $M$ линейно связно.

Отметим следующий общий результат [203; лемма 1].

Пусть $X$ - банахово пространство. Тогда

$$
\left(\stackrel{\circ}{B}_{l}\right) \cap(\mathscr{F})=(\stackrel{\circ}{B}) \cap(\mathscr{F}) .
$$

Таким образом, в банаховом пространстве каждое замкнутое $\stackrel{\circ}{B}$-связное множество линейно связно. Для незамкнутых множеств это неверно даже в $X=\mathbb{R}^{2}$ (соответствующий пример построен Е. В. Щепиным, см. [203; § 2]). 
Остановимся на вопросе о связности чебышёвских множеств. Исследование связности чебышёвских множеств началось в 1960-х годах с работ Д. Вулберта [226], [227], Й. Блаттера, Д. Вулберта, П. Морриса [47] и Л. П. Власова [220] и было продолжено Б. Брозовским, Ф. Дойчем [68], [67], Л. П. Власовым [222], Ч. Данхемом [93], С. В. Конягиным [138], В. Кли [135], [136], С. Пападополу [174], И. Г. Царьковым [203], [207], В. А. Кощеевым [148], А. Р. Алимовым [15], [17], П. А. Бородиным [52] и другими.

При исследовании связности чебышёвских множеств Вулберт [226], [227] ввел

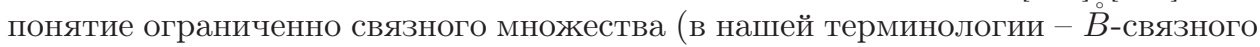
множества) и установил [227], что в произвольном банаховом пространстве чебышёвское множество с непрерывной метрической проекцией является $\stackrel{\circ}{B}$-связным (ср. с теоремой 5.1). Это утверждение было уточнено Блаттером, Моррисом и Вулбертом [47; теорема 14], которые установили, что P-компактное множество существования с $H$-непрерывной (непрерывной по Хаусдорфу) метрической проекцией в линейном нормированном пространстве $\stackrel{\circ}{B}$-связно и $P$-связно. В. Поллул [178; с. 56, теорема 2] усилил этот результат, показав, что в линейном нормированном пространстве множество существования $M$ с непрерывной (т. е. полунепрерывной одновременно сверху и снизу) метрической проекцией $\stackrel{\circ}{B}$-связно и $P$-связно (в случае аппроксимативной компактности такое $M$ является $B$-связным (теорема 5.4)). В дальнейшем условие непрерывности $P_{M}$ было несколько ослаблено Брозовским и Дойчем [68] (см. теорему 5.5 ниже).

Пусть $M \subset X$ и $x_{0} \in X$. Напомним, что метрическую проекцию $P_{M}$ называют ORU-непрерывной ("outer radially upper continuous") в точке $x_{0}$ (см. [66], [68]), если для любого $y_{0} \in P_{M} x_{0}$ и любого открытого $W \supset P_{M} x_{0}$ найдется окрестность $U$ точки $x_{0}$ такая, что $P_{M} x \subset W$ при любом $x \in U \cap\left\{y_{0}+\lambda\left(x_{0}-y_{0}\right) \mid \lambda \geqslant 1\right\}$. Ясно, что полунепрерывность сверху влечет ORU-непрерывность.

Метрическая проекция $P_{M}$ называется IRL-непрерывной (от англ. "inner radially lower continuous") в точке $x_{0}$, если для любого $y_{0} \in P_{M} x_{0}$ и любого открытого $W$ такого, что $W \cap P_{M} x_{0} \neq \varnothing$, найдется окрестность $U$ точки $x_{0}$ такая, что $P_{M} x \cap W \neq \varnothing$ при любом $x \in U \cap\left\{y_{0}+\lambda\left(x_{0}-y_{0}\right) \mid 0 \leqslant \lambda \leqslant 1\right\}$. Ясно, что из полунепрерывности снизу вытекает IRL-непрерывность. Отметим, что если $M$ выпукло или является чебышёвским множеством, то метрическая проекция $P_{M}$ является IRL-непрерывной [68; следствие 3.4].

Следующий результат установлен Брозовским и Дойчем [68].

Tеорема 5.5. Пусть $M$-множество существования, для которого метрическая проекиия IRL- и ORU-непрерьвна. Тогда $M$ является $\stackrel{\circ}{B}$-связным и Р-связным.

Метрическая проекция на чебышёвское солнце IRL- и ORU-непрерывна [68]. Как следствие, имеет место приводимая ниже теорема, первое утверждение которой установлено Брозовским и Дойчем [68; следствие 5.3], а второе вытекает из первого с учетом теоремы 5.4 .

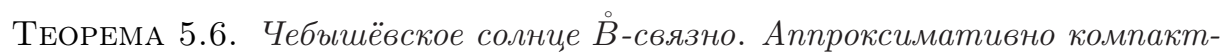
ное чебышёвское солние В-связно.

Отметим следующий результат (Вулберт [227; теорема 3]). 
Теорема 5.7. Для локально компактного чебишёвского множества $M$ в банаховом пространстве следующие свойства эквивалентны:

а) метрическая проекиия $P_{M}$ непрерывна;

ь) $M$ является $\stackrel{\circ}{B}$-связным;

c) $M-$ солние.

Следующие два результата установлены Царьковым [206], [203]. В частности, из теоремы 5.9 и равенства (5.1) следует (см. [203]), что в пространстве Ефимова-Стечкина всякое чебышёвское множество линейно связно.

Теорема 5.8. Локалъно компактное множество единственности (в частности, локально компактное чебышёвское множество) в гладком пространстве Ефимова-Стечкина выпукло.

Теорема 5.9. Имеют место следующие утверждения:

$$
\begin{aligned}
& X \in(\mathrm{ES}) \quad \Longrightarrow \quad\left(P_{0}\right) \cap(\mathscr{F}) \subset(\stackrel{\circ}{B}) ; \\
& X \in(\mathrm{LUR}) \cap(\mathrm{Rf})[=(\mathrm{ES}) \cap(\mathrm{R})] \quad \Longrightarrow \quad\left(P_{0}\right) \subset(B) ; \\
& X \in(\mathrm{CLUR}) \Longrightarrow\left(P_{0}\right) \cap(\stackrel{\circ}{B}) \cap(\mathscr{F})=(B) \cap(\mathscr{F}) ; \\
& X \in(\mathrm{Rf}) \cap(\mathrm{CLUR})[\subset(\mathrm{ES})] \quad \Longrightarrow \quad\left(P_{0}\right) \cap(\mathscr{F})=(B) \cap(\mathscr{F}) .
\end{aligned}
$$

Из отрицательных результатов отметим следующие. Если $X \notin(\mathrm{Rf})$, то

$$
\begin{aligned}
& \left(P_{0}\right) \cap \mathscr{F} \not \subset(\stackrel{\circ}{B}), \\
& \left(P_{0}\right) \cap \mathscr{F} \neq(B) \cap(\mathscr{F}) .
\end{aligned}
$$

Для доказательства первого утверждения достаточно рассмотреть пример 4.1 (дополнение к антипроксиминальной гиперполосе). Второе утверждение установлено в [203].

Ч. Данхем [93] построил пример несвязного чебышёвского множества в $C[0,1]$ (множество в примере Данхема локально компактно, но не аппроксимативно компактно и не является солнцем). В. Кли [135], [136] построил пример дискретного чебышёвского множества в пространстве $l^{1}(\mathfrak{n})$, где $\mathfrak{n}$ - бесконечный регулярный кардинал такой, что $\mathfrak{n}^{\aleph_{0}}=\mathfrak{n}$. Множество в примере Кли также не является солнцем (солнце не может иметь собственных изолированных точек) и не является $\delta$-солнцем. Пример дискретного чебышёвского множества в сепарабельном метрическом пространстве построен С. Пападополу [174]. Стоит также отметить, что известный пример Джонсона невыпуклого ограниченного чебышёвского множества в предгильбертовом пространстве $l_{0}^{2}$ (см. [32; $\S 2],[127]$, [87]) может быть видоизменен таким образом, что полученное чебышёвское множество будет "несвязной пеной” (Джонсон [129]).

Среди нерешенных проблем в этой области выделим следующие.

Провлема 5. Охарактеризовать (бесконечномерные) пространства, в которых каждое чебышёвское множество связно.

ПроблемА 6. Охарактеризовать (бесконечномерные) пространства, в которых чебышёвское множество с непрерывной метрической проекцией является солнцем. (В частности, в каких пространствах аппроксимативно компактное чебышёвское множество является солнцем?) 
Положительные результаты в этом направлении содержатся в работах Браесса [57], Брозовского и Дойча [69]. $\mathrm{K}$ примеру, в $C(Q)$ чебышёвское множество с непрерывной метрической проекцией (в частности, аппроксимативно компактное) является солнцем [57]. О чебышёвских множествах в пространствах непрерывных функций см. также [92], [57], [9], [10].

В связи с проблемой 6 отметим следующий результат Власова [220] (см. также [132]): в банаховом пространстве локально компактное чебышёвское множество с непрерывной метрической проекцией является солнцем.

\section{6. Понятия ацикличности и клеточноподобности множеств}

Понятия ацикличности и клеточноподобности оказываются важными для изучения аппроксимативных свойств множеств, в частности их солнечности. В данном вспомогательном разделе мы напоминаем некоторые понятия из геометрической топологии и, в частности, обсуждаем понятия ацикличности и клеточноподобности. Ацикличность возникает здесь в связи со следующей прямой теоремой геометрической теории приближений (Власов [222]):

в банаховом пространстве Р-ацикличное ограниченно компактное множество является солнцем.

Ответ на обратный вопрос в общем случае не известен (см. раздел 9). Гипотеза состоит в том, что ацикличность (Р-ацикличность) здесь "по делу"; иными словами, ацикличность является свойством, присущим всем солнцам в конечномерных пространствах (это заведомо так в двумерных пространствах, пространстве $\ell^{\infty}(n)$ и, конечно, в гладких пространствах).

Теория гомологий (когомологий) связывает с каждым топологическим пространством $X$ последовательности абелевых групп $H_{k}(X), k=0,1,2, \ldots$ (группы гомологий), и $H^{k}(X), k=0,1,2, \ldots$ (группы когомологий), которые являются гомотопическими инвариантами пространства: если два пространства гомотопически эквивалентны, то и соответствующие группы гомологий изоморфны. Группы (ко)гомологий можно конструировать различными способами (имеется, например, конструкция с использованием нервов покрытий, предложенная П. С. Александровым и обобщенная Э. Чехом; конструкция Л. Вьеториса, основанная на понятии истинных циклов; конструкция, основанная на понятии сингулярных цепей).

Пусть $A$ - произвольная нетривиальная абелева группа. Метризуемое пространство называется ацикличным, если его группа чеховских когомологий с коэффициентами из $A$ тривиальна. Таким образом, определение ацикличности зависит от выбранной группы коэффициентов. Отметим, что гомологии (Александрова-)Чеха не образуют теории гомологий, поскольку не удовлетворяют аксиоме точности, а когомологии Чеха образуют теорию гомологий топологических пространств. С подробным изложением теории (ко)гомологий компактов, топологических и равномерных пространств можно ознакомиться в обзоре С. А. Мелихова [165].

Если гомология (когомология) имеет компактный носитель (т. е. удовлетворяет аксиоме компактных носителей) и коэффициенты группы гомологий (когомологий) лежат в поле, то понятия гомологической и когомологической ацикличности совпадают [162]. Однако в случае произвольной абелевой группы коэффициентов понятия гомологической и когомологической ацикличности могут быть различны (см., например, [95]). 
Ниже, если не оговорено противное, ацикличность будет пониматься относительно чеховских когомологий с коэффициентами в произвольной абелевой группе.

Непустое компактное пространство называется $R_{\delta}$-множеством (см., например, [114; формула (2.11)]), если оно гомеоморфно пересечению счетной убывающей последовательности абсолютных компактных ретрактов (или стягиваемых компактов [114; теорема 2.13]). $R_{\delta}$-множества естественно возникают как пространства решений задачи Коши для неавтономных и автономных дифференциальных уравнений и включений [113], [90], [27]. Результаты такого типа восходят к Н. Ароншайну, который установил $R_{\delta}$-свойство для множества локальных решений задачи Коши в конечномерном пространстве $\mathbb{R}^{n}$.

Компакт $Y$ называется клеточноподобным (или имеющим шейп точки), если существуют абсолютный окрестностный ретракт $Z$ и вложение $i: Y \rightarrow Z$ такие, что образ $i(Y)$ стягиваем в любой своей окрестности $U \subset Z$ (см. [114; формула (82.4)]); само клеточноподобное множество при этом не обязано быть стягиваемым. Топологическое пространство $X$ стягиваемо (в точку), если тождественное отображение этого пространства в себя гомотопно отображению в точку. Из известной характеризации Д. Химана $R_{\delta}$-множеств непосредственно следует, что $R_{\delta}$-множество всегда клеточноподобно $[151 ; \S 4.2]$, [123; с. 50]. Поскольку всякое отображение компакта точечного шейпа в абсолютном окрестностном ретракте гомотопически тривиально, то компакт шейпа точки (клеточноподобный) стягивается во всякой своей окрестности в любом объемлющем абсолютном окрестностном ретракте. Как следствие, классы $R_{\delta}$-множеств $u$ клеточноподобных (иейпа точки) компактов совпадают.

Отметим, что клеточноподобность влечет ацикличность (относительно любой непрерывной теории (ко)гомологий) [151; с. 854], при этом имеются примеры ацикличных, но не клеточноподобных множеств, а также примеры клеточноподобных, но не линейно связных множеств (синусоида топологов).

\section{7. Выборки из оператора наилучшего и почти наилучшего приближения. Солнечность, ретракция}

Хорошо известно, что оператор наилучшего приближения обладает недостаточной устойчивостью даже при приближении чебышёвскими подпространствами, не говоря уже о нелинейных множествах. К примеру, в классическом случае приближения многочленами $\mathscr{P}_{n}$ в $C[0,1]$ оператор метрической проекции не является равномерно непрерывным на единичном шаре. Более того, давно известны примеры чебышёвских подпространств с разрывной метрической проекцией (Е. В. Ошман, А. Браун, В. И. Андреев и др.); в $C[0,1]$ метрическая проекция на множество дробно-рациональных функций $\mathscr{R}_{m, n}$ и множество экспоненциальных сумм $E_{n}^{+}$имеет точки разрыва. Для исправления такой ситуации был предложен способ повышения устойчивости приближения за счет сопоставления подходящим образом приближаемому элементу одного из его почти наилучших приближений. Так появилось понятие $\varepsilon$-выборки ( $\varepsilon$-селекции).

Вопросами существования непрерывной $\varepsilon$-выборки и устойчивости оператора почти наилучшего приближения для классических объектов теории приближений занимались Д. Вулберт, О. А. Лисковец, В. И. Бердышев, С. В. Конягин, 
А. В. Маринов, И. Г. Царьков, П. В. Альбрехт, К. С. Рютин, Е. Д. Лившиц и др. Стоит подчеркнуть, что вопрос об устойчивости оператора почти наилучшего приближения возникает не только в самой теории приближений и численных методах, но и в некорректных задачах, оптимальном управлении, математическом программировании и при исследовании устойчивости решений общих экстремальных задач. Обзор современного состояния этой области дан в работе Д. Реповша и П. В. Семенова [181].

Пусть $F: D \rightarrow 2^{X}$. Отображение $\varphi: D \rightarrow 2^{X}$ называется селекцией (выборкой) $F$, если $\varphi(x) \subset F(x)$ для любого $x \in D$ и из того, что $F(x) \neq \varnothing$, следует, что $\varphi(x) \neq \varnothing$.

Мы будем рассматривать как однозначные, так и многозначные выборки.

Начнем со случая выборок из метрической проекции. Приводимые ниже теоремы 7.1-7.4 получены Царьковым [207].

Теорема 7.1. В банаховом пространстве множество с полунепрерывной снизу выборкой из метрической проекиии $P_{0}$-связно.

ТеОРема 7.2. В линейном нормированном пространстве Р-связное множество существования с полунепрерывной снизу выборкой из метрической проекиии В-связно.

Теорема 7.3. Пусть $X$ - конечномерное банахово пространство, и пусть $M \subset X-$ множество существования с полунепрерывной снизу метрической проекиией. Тогда $M$ является $B$-аиикличным.

Теорема 7.4. В банаховом пространстве множество существования с полунепрерывной снизу выборкой из метрической проекиии В-связно.

Пусть $\varepsilon>0, M \subset X$. Отображение $\varphi: X \rightarrow M$ называют мультипликативной (аддитивной) $\varepsilon$-выборкой, если для всех $x \in X$ имеет место неравенство

$$
\|x-\varphi(x)\| \leqslant(1+\varepsilon) \rho(x, M) \quad(\text { соответственно }\|x-\varphi(x)\| \leqslant \rho(x, M)+\varepsilon) .
$$

Из классической теоремы Майкла о селекции следует, что для всех $\varepsilon>0$ существует непрерывная мультипликативная (аддитивная) $\varepsilon$-выборка на любое выпуклое замкнутое подмножество банахова пространства.

В качестве примера невыпуклого множества, обладающего непрерывной аддитивной (мультипликативной) $\varepsilon$-выборкой для всех $\varepsilon>0$, можно указать единичную сферу в бесконечномерном линейном нормированном пространстве.

Далее обсудим задачу о характеризации множеств, допускающих непрерывную $\varepsilon$-выборку для любого числа $\varepsilon>0$. Нам потребуются следующие определения.

Множество $A$ в полуметрическом пространстве $(Y, \nu)$ называем бесконечно связным, если для всех $n \in \mathbb{N}$, единичного шара $B \subset \mathbb{R}^{n}$ и произвольного непрерывного отображения $\varphi: \operatorname{bd} B \rightarrow A$ существует непрерывное продолжение $\widetilde{\varphi}: B \rightarrow A$.

Множество $M \subset X$ называется $\stackrel{\circ}{B}$-бесконечно связным, если пересечение множества $M$ с любым открытым шаром либо пусто, либо бесконечно связно.

Отметим, что $\stackrel{B}{B}$-бесконечно связное множество не обязано быть $B$-бесконечно связным, т. е. пересечение с некоторым замкнутым шаром может быть не 
бесконечно связным и даже несвязным (см. [203; теорема 5]). Отметим также, что $\stackrel{B}{B}$-бесконечно связным и даже $B$-бесконечно связным множеством является такой классический объект, как множество обобщенных рациональных дробей в пространстве непрерывных функций $C(Q)$.

Для $M \subset X$ и произвольного $\delta>0$ положим

$$
\begin{aligned}
& \stackrel{\circ}{P_{M}^{\delta}} x:=\{y \in M \mid\|x-y\|<\rho(x, M)+\delta\}, \\
& P_{M}^{\delta} x:=\{y \in M \mid\|x-y\| \leqslant \rho(x, M)+\delta\} .
\end{aligned}
$$

Подмножество $A \subset X$ называется ретрактом множества $X$, если существует непрерывное отображение $r: X \rightarrow A$ (называемое ретракцией) такое, что $\left.r\right|_{A}=\left.1\right|_{A}$, т. е. тождественное отображение $1_{A}$ допускает непрерывное продолжение на все пространство $X$. Хорошо известно, что в конечномерном пространстве граница шара не является ретрактом шара. Однако также хорошо известно, что в любом бесконечномерном пространстве для любого $\varepsilon>0$ существует липшицева $\varepsilon$-выборка из шара на сферу, при этом сфера также является липшицевым ретрактом шара.

Маринов [159] рассмотрел ряд вопросов о мере несвязности и величине разрывов оператора $\delta$-проекции $\left(P_{M}^{\delta}\right)$ и охарактеризовал аппроксимирующие множества с непрерывной $\delta$-проекцией. В частности, он показал, что в равномер-

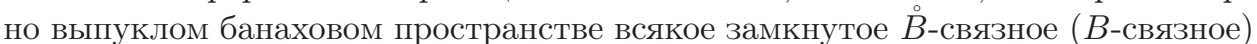
множество обладает устойчивыми проекторами $P_{M}^{\delta} x$ и $\stackrel{\circ}{P}_{M}^{\delta} x$ по параметрам $x$ и $\delta>0$. Из этих результатов следует, что для $\stackrel{\circ}{B}$-бесконечно связных замкнутых множеств в равномерно выпуклых пространствах оператор $P_{M}^{\delta} x$ устойчив.

Множество $A$ в полуметрическом пространстве $(Y, \nu)$ называется аппроксимативно бесконечно связным, если для всех $n \in \mathbb{N}$, единичного шара $B \subset \mathbb{R}^{n}$, произвольного непрерывного отображения $\varphi: \operatorname{bd} B \rightarrow A$ и любого $\varepsilon>0$ существует $\varepsilon$-продолжение для отображения $\varphi$, т. е. такое непрерывное отображение $\varphi_{\varepsilon}: B \rightarrow A$, для которого $\left\|\varphi(x)-\varphi_{\varepsilon}(x)\right\|<\varepsilon(x \in \operatorname{bd} B)$.

Приводимые ниже теоремы 7.5-7.10 установлены Царьковым [214].

Теорема 7.5. Пусть $(X,\|\cdot\|)$ - линейное полунормированное пространство и $M \subset X$ непусто. Тогда следующие условия вытекают друг из друга последовательно:

а) множество $M$ является $\stackrel{\circ}{B}$-бесконечно связным;

b) для любой полунепрерьвной снизу функции $\psi: X \rightarrow \overline{\mathbb{R}}$ такой, что $\psi(x)>$ $\varrho(x, M)(x \in X)$, существует $\varphi \in C(X, M)$ такая, ито

$$
\|\varphi(x)-x\|<\psi(x) \quad(x \in X)
$$

c) для любого числа $\varepsilon>0$ существует $\varphi \in C(X, M)$ такая, что

$$
\|\varphi(x)-x\|<\varrho(x, M)+\varepsilon \quad(x \in X)
$$

(т.е. непрерывная аддитивная в-выборка); 
d) множество $M$ является $\stackrel{\circ}{B}$-аппроксимативно бесконечно связным, m.е. непустое пересечение множества $M$ и произвольного шара $\stackrel{\circ}{B}(x, r)$ является аппроксимативно бесконечно связным.

ТЕОРема 7.6. Пусть $(X,\|\cdot\|)$ - полное линейное полунормированное пространство, а $M \subset X$ непусто и замкнуто. Тогда следующие условия равносильны:

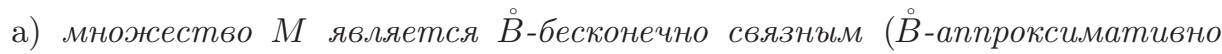
бесконечно связным);

b) для любой полунепрерьвной снизу функции $\psi: X \rightarrow \overline{\mathbb{R}}$ такой, что $\psi(x)>$ $\varrho(x, M)(x \in X)$, существует $\varphi \in C(X, M)$ такал, что

$$
\|\varphi(x)-x\|<\psi(x) \quad(x \in X) ;
$$

c) для любого числа $\varepsilon>0$ существует $\varphi \in C(X, M)$ такая, что

$$
\|\varphi(x)-x\|<\varrho(x, M)+\varepsilon \quad(x \in X)
$$

(т.е. непрерывная аддитивная в-выборка);

d) для любой полунепрерьвной снизу функции $\theta: X \rightarrow \overline{\mathbb{R}}_{+}$такой, что $\theta>1$, существует отображсение $\varphi \in C(X, M)$ такое, что

$$
\|\varphi(x)-x\| \leqslant \theta(x) \varrho(x, M) \quad(x \in X) ;
$$

е) для любого числа $\varepsilon>0$ существует $\varphi \in C(X, M)$ такая, что

$$
\|\varphi(x)-x\| \leqslant(1+\varepsilon) \varrho(x, M) \quad(x \in X)
$$

(т.е. непрерывная мультипликативная в-выборка);

f) для любъх $x \in X$ u $R>0$ maкux, что $N=M \cap \stackrel{\circ}{B}(x, R) \neq \varnothing$, множество $N$ является ретрактом шара $\stackrel{\circ}{B}(x, R)$;

g) для любых $x \in X$ u $R>0$ maкux, что $N=M \cap \stackrel{\circ}{B}(x, R) \neq \varnothing$, множество $N$ стягиваемо по себе в точку.

ТЕорема 7.7. Пусть $(X,\|\cdot\|)$ - линейное полунормированное пространство, а $M \subset X$ непусто и замкнуто. Тогда следующие условия равносильны:

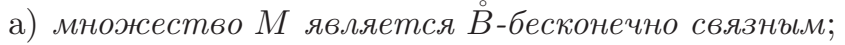

b) для любой полунепрерьвной снизу функции $\psi: X \rightarrow \overline{\mathbb{R}}$ такой, что $\psi(x)>$ $\varrho(x, M)(x \in X)$, существует $\varphi \in C(X, M)$ такая, что

$$
\|\varphi(x)-x\|<\psi(x) \quad(x \in X) ;
$$

с) для любой полунепрерьвной снизу функции $\theta: X \rightarrow \mathbb{R}$ такой, что $\theta(x)>1$ $(x \in X)$, существует отображение $\varphi \in C(X, M)$ такое, что

$$
\|\varphi(x)-x\| \leqslant \theta(x) \varrho(x, M) \quad(x \in X) ;
$$

d) для любого числа $\varepsilon>0$ существует $\varphi \in C(X, M)$ такая, что

$$
\|\varphi(x)-x\| \leqslant(1+\varepsilon) \varrho(x, M) \quad(x \in X)
$$

(т.е. непрерывная мультипликативная в-выборка);

е) для любых $x \in X u R>0$ maких, что $N=M \cap \stackrel{\circ}{B}(x, R) \neq \varnothing$, множество $N$ стягиваемо по себе в точку. 
Эти утверждения позволяют для известных и новых аппроксимирующих нелинейных множеств (в основном в пространстве $C(Q))$ доказывать существование непрерывной $\varepsilon$-выборки как для малых, так и для конкретных $\varepsilon$ (зависящих от параметров множества). Без доказательства отметим, что аппроксимативно компактное монотонно линейно связное множество $\stackrel{B}{B}$-бесконечно связно, является $\delta$-солнцем и обладает непрерывными $\varepsilon$-выборками для всех $\varepsilon>0$ (см. теорему 9.6). K примеру, аналогичное утверждение верно для ломаных с нефиксированными узлами (Лившиц [154]), для обобщенных дробно-рациональных функций в $C(Q)$ (см. [25; замечание 6.1]) и для множества конечных экспоненциальных сумм (с ограничением или без ограничения на коэффициенты) в $C[0,1]$.

СЛЕДСТВИЕ 7.1. Пусть множество $M$ обладает непрерывной аддитивной $\varepsilon$-выборкой для любого $\varepsilon>0$. Тогда на любую $r$-окрестность $M$ есть непрерывная аддитивная в-выборка для любого $\varepsilon>0$. Следовательно, окрестность $O_{r}(M)$ является $\stackrel{\circ}{B}$-бесконечно связным множеством, а $M$ является $\stackrel{\circ}{B}$-аппроксимативно бесконечно связным множеством, и в случае, когда $M$ обладает непрерывной мультипликативной $\varepsilon$-выборкой для любого $\varepsilon>0$, оно является $\stackrel{\circ}{B}$-бесконечно связным и даже $\stackrel{\circ}{B}$-стягиваемым.

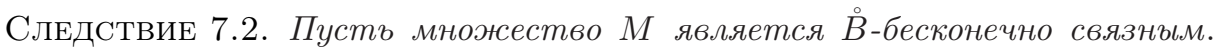

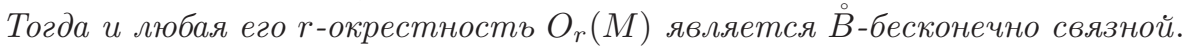

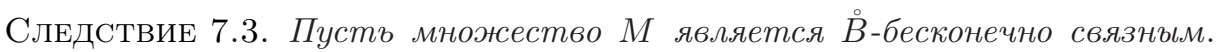
Тогда его замыкание является $\stackrel{\circ}{B}$-стягиваемым.

В задаче о связи локальных и глобальных непрерывных $\varepsilon$-выборок, а также устойчивости их по параметру $\varepsilon$ имеют место следующие результаты.

Теорема 7.8. Пусть $M$ - замкнутое множество в полном линейном полунормированном пространстве $(X,\|\cdot\|)$, обладающее для любого $\varepsilon>0$ непрерывной аддитивной в-выборкой. Тогда существует отображение $f \in C(X \times$ $(0,+\infty), M)$ такое, что $f(\cdot, \varepsilon)$ является непрерывной аддитивной $\varepsilon$-выборкой для $M$.

СЛЕДСТВИЕ 7.4. Пусть $M$ - замкнутое множество в полном линейном полунормированном пространстве $(X,\|\cdot\|)$ и в некоторой окрестности $O(x)$ произвольной точки $x \in X$ для любого $\varepsilon>0$ существует непрерывная аддитивная в-выборка на $M$. Тогда $M$ обладает непрерывной аддитивной (мультипликативной) $\varepsilon$-выборкой для всех $\varepsilon>0$.

В качестве примера множеств, обладающих непрерывной $\varepsilon$-выборкой, можно рассмотреть аппроксимативно компактные монотонно линейно связные множества (подробнее см. раздел 9).

ТеОрема 7.9. Пусть $M$ - аппроксимативно компактное и монотонно линейно связное множество в банаховом пространстве $(X,\|\cdot\|)$. Тогда на $M$ существует непрерывная аддитивная в-выборка.

В следующем утверждении дается ответ вопрос о существовании непрерывных выборок для множества векторозначных ограниченных непрерывных функций с образами в множестве, обладающем непрерывной выборкой. 
ТЕОРема 7.10. Пусть множество $M$ в полном линейном полунормированном пространстве $(X,\|\cdot\|)$ обладает непрерьвной аддитивной (мультипликативной) $\varepsilon$-выборкой для некоторого $\varepsilon>0$. Тогда множество $B C(Q, M)$ в пространстве $B C(Q, X)$ обладает непрерывной аддитивной (мультипликативной) в-въборкой.

Здесь $B C(Q, X)$ - пространство всех ограниченных и непрерывных функций из $Q$ в $X$ с равномерной нормой.

Из доказательства теоремы 7.6 вытекает следующий аналог известного утверждения о том, что $B$-связное множество является $\stackrel{\circ}{B}$-связным (см., например, [222; предложение 0.5]).

ТЕОрема 7.11. В-стягиваемое множество является $\stackrel{\circ}{B}$-стягиваемым.

Было бы интересно получить обратный результат к теореме 7.11 (по типу теоремы 5.4 , утверждающей, что $(\mathrm{AC}) \cap(\stackrel{\circ}{B}) \subset(B))$.

Следующее утверждение (Царьков [208; теорема 1]) обобщает один результат Власова [222; теорема 4.16], рассматривавшего в данной ситуации $P$-компактные $P$-выпуклые множества.

Теорема 7.12. Пусть $X$ - банахово пространство и $M-P$-ацикличное множество с Н-полунепрерывной сверху метрической проекицей. Тогда $M$ почти выпукло (является $\delta$ - и $\gamma$-солни,м ).

Отсюда и из теоремы 7.6 вытекает (см. Царьков [208]), что если в банаховом пространстве $X$ дано аппроксимативно компактное множество $M$, обладающее непрерывной (аддитивной) $\varepsilon$-выборкой при любом $\varepsilon>0$, то $M$ является $P$-ацикличным и почти выпуклым. Если вдобавок $X$ принадлежит (SS) (т.е. является сильно гладким в смысле Власова [219]; см. определение на с. 19) или $X^{*}$ строго выпукло, то $M$ выпукло.

Из теоремы 7.12 вытекает следующее утверждение. Пусть $n \in \mathbb{N}, m \in \mathbb{Z}_{+}$, $p \in \mathbb{R}, 1<p<\infty$. Тогда в $L^{p}$ найдется элемент, для которого множество ближайших элементов в множестве рациональных дробей $\mathscr{R}_{m, n}$ компактно и не ациклично [208].

Приводимые ниже теоремы 7.13-7.15 и их следствия установлены Царьковым [209], [211]. Отметим, что здесь рассматриваются многозначные $\varepsilon$-выборки.

ТеОрема 7.13. Пусть $M$ - аппроксимативно компактное подмножество банахова пространства $X$, обладающее для любого $\varepsilon>0$ полунепрерывной сверху ацикличной аддитивной в-выборкой из метрической проекции на себя относительно некоторого всюду плотного выпуклого подмножества пространства $X$. Тогда множество $M$ является $P$-ацикличным.

Теорема 7.14. Пусть банахово пространство $X$ таково, что $X \in(\mathrm{SS})$ или $X^{*} \in(\mathrm{R})$, и пусть $M \subset X$ аппроксимативно компактно и невыпукло. Тогда для любого выпуклого подмножества $L \subset X$, всюду плотного в $X$, найдется точка $x \in L$ такая, что множество $P_{M} x$ не ациклично.

СлЕДСТвИЕ 7.5. Пусть банахово пространство $X$ таково, что $X \in(\mathrm{SS})$ или $X^{*} \in(\mathrm{R})$, и пусть $M$ - аппроксимативно компактное $P$-ацикличное подмножество $X$. Тогда $M$ выпукло. 
СлеДСтвиЕ 7.6. Пусть $H$ - гилъбертово пространство, а множество $M \subset H$ аппроксимативно компактно и невыпукло. Тогда для любого выпуклого подмножества $L \subset H$, всюду плотного в $H$, найдется точка $x \in L$ такая, что множество $P_{M} x$ не ациклично (причем в случае $\operatorname{dim} H=\infty$ таких точек бесконечно много).

Интересно отметить применение теорем 7.13, 7.14 в теории обучения при построении оценщика (Ю. В. Малыхин [157]).

В [209] с помощью теоремы 7.14 и вариационного принципа доказана неединственность ненулевых решений в классе $\stackrel{\circ}{W}_{2}^{1}(\Omega)$ в задаче Дирихле

$$
\left\{\begin{array}{l}
\Delta u=\gamma u+F(x, u)+\varphi(x), \\
u=0 \text { на bd } \Omega
\end{array}\right.
$$

для некоторой бесконечно дифференцируемой функции $\varphi$, носитель которой лежит в ограниченной замкнутой области $\Omega \subset \mathbb{R}^{n}$ (см. также работы Б. Ричери [184], Ф. Фарачи, А. Ианидзотто [98], Б. Е. Брекнер, А. Хорвас, Ц. Варга [60] и др.).

Теорема 7.15. Пусть $X$ - банахово пространство, $M \subset X, x \in \mathrm{AC}(M) \backslash M$. Предположим, что найдется окрестность $O_{r}(x)$, для которой существует полунепрерывная сверху ацикличная в-выборка $\varphi_{\varepsilon}: O_{r}(x) \rightarrow 2^{M}$ при любом $\varepsilon>0$. Тогда $x$ - точка $\delta$-солнечности для $M$. При этом, если $X \in(\mathrm{R})$, то $P_{M} x$ состоит из одной точки.

Из теорем 4.3 и 7.15 вытекает, что аппроксимативно компактное множество, обладающее непрерывной $\varepsilon$-выборкой для всех $\varepsilon>0$, является в пространстве $X \in(\mathrm{LUR})$ чебышёвским солнцем.

Из теоремы 7.15 вытекает, что если $X \in(\mathrm{B}) \cap(\mathrm{SS})$ или $X^{*} \in(\mathrm{R})$, а $M-$ аппроксимативно компактное подмножество $X$, обладающее для любого $\varepsilon>0$ полунепрерывной сверху ацикличной аддитивной $\varepsilon$-выборкой на $X \backslash M$, то $M$ выпукло. Также из теоремы 7.15 вытекает, что если $X \in(\mathrm{R})$, а $M$ - аппроксимативно компактное подмножество $X$, обладающее для любого $\varepsilon>0$ полунепрерывной сверху ацикличной аддитивной $\varepsilon$-выборкой, то $M$ - чебышёвское множество. В [211] также доказывается, что если для $X \in(\mathrm{R})$ последнее условие выполнено на некотором выпуклом множестве $N$ таком, что $X \backslash M \subset \bar{N}$, то $M$ - чебышёвское множество.

СлеДСтвие 7.7. Пусть $X \in(\mathrm{R})$, и пусть $M \subset X$ - аппроксимативно компактное подмножество, обладающее полунепрерывной сверху ацикличной $\varepsilon$-выборкой на окрестности $O(x)$ для любого $\varepsilon>0$. Тогда метрическая проекция $P_{M}$ у одноточечна для всех $y \in O(x)$.

СлеДСтвиЕ 7.8. Пусть $X \in(\mathrm{R})$, и пусть $M \subset X$ - аппроксимативно компактное подмножество, обладающее полунепрерывной сверху ацикличной

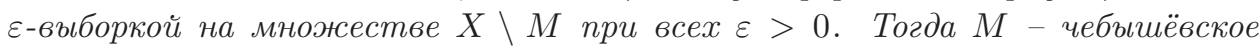
множество.

Отображение $f: N \rightarrow X$ называют $\varepsilon$-сдвигом, если $\|f(x)-x\| \leqslant \varepsilon$ для всех $x \in N$. Теоремы 7.16, 7.17 содержатся в [211]. 
Tеорема 7.16. Пусть $X$ - банахово пространство, $M \subset X, x \in \mathrm{AC}(M) \backslash M$, $O(x) \subset X$ - некоторая окрестность точки $x$ и для любого $\varepsilon>0$ существуют множество $N_{\varepsilon} \subset X$ и непрерывный $\varepsilon$-сдвиг $f_{\varepsilon}: O(x) \rightarrow N_{\varepsilon}$. Предположим также, что на множестве $N_{\varepsilon}$ существует полунепрерывная сверху ацикличная $\varepsilon$-выборка $\psi_{\varepsilon}: N_{\varepsilon} \rightarrow M$. Тогда $x$ - точка $\delta$-солнечности, и если дополнительно $X \in(\mathrm{R})$, то $P_{M} x$ состоит из одной точки.

Tеорема 7.17. Пусть $X-$ банахово пространство, $M \subset X, x \in \mathrm{AC}(M) \backslash M$, $O(x) \subset X$ - некоторая окрестность точки $x$ и для любого $\varepsilon>0$ существует выпуклое множество $K_{\varepsilon} \subset X, d\left(K_{\varepsilon}, O(x)\right)<\varepsilon$, на котором существует

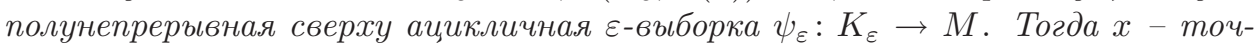
ка $\delta$-солнечности и если дополнительно $X \in(\mathrm{R})$, то $P_{M} x$ состоит из одной точки.

СлеДСтвиЕ 7.9. Пусть $X \in(\mathrm{R})$, и пусть $M \subset X$ - аппроксимативно компактное подмножество, обладающее для всех $\varepsilon>0$ полунепрерывной сверху ацикличной в-выборкой на некотором выпуклом множестве $N$ таком, что $X \backslash M \subset \bar{N}$. Тогда $M-$ чебышёвское множество.

ЗАмЕчАниЕ 7.1. Доказательство теорем 7.15-7.17 опирается на известную теорему Эйленберга-Монтгомери о неподвижной точке (см., например, [114; следствие (32.12)]) и теорему Вьеториса-Бегла об отображении. При этом ацикличность понимается в смысле групп гомологий (Александрова-)Чеха с коэффициентами в поле (в работе Эйленберга-Монтгомери ацикличность понимается в смысле циклов Вьеториса и групп гомологий над полем коэффициентов). Однако гомологии Чеха изоморфны гомологиям Вьеториса на категории компактных метризуемых пространств, а понятия гомологической и когомологической ацикличности совпадают, если коэффициенты группы гомологий (когомологий) лежат в поле и носитель компактен.

Теперь рассмотрим применение ретракции в теории приближений. Хорошо известно, что ретракции являются важным инструментом в теоремах о неподвижных точках. K примеру, классическая теорема Брауэра о неподвижной точке эквивалентна утверждению об отсутствии непрерывной ретракции конечномерного шара на его границу.

Начнем со следующего геометрически ясного результата. Пусть $M$ - чебышёвское множество с непрерывной метрической проекцией в линейном нормированном пространстве $X$ и $M \cap B(x, r) \neq \varnothing$ при некоторых $x \in X$ и $r>0$. Тогда

$$
B(x, r) \cap M \text { есть ретракт шара } B(x, r) .
$$

Требуемая ретракция устроена просто: для $z \in B(x, r)$ шар $B(x, r)$ гомотетично сдувается относительно центра гомотетии $z$ до тех пор, пока не получается шар $B_{z}$, касающийся $M$. Тогда $B_{z} \cap M$ - это точка $\varphi(z)$. Отображение $\varphi: B(x, r) \rightarrow B(x, r) \cap M$ является требуемой ретракцией.

Имеет место следующий результат ([202], см. также [72]).

Лемма 7.1. Пусть $X$ - конечномерное линейное нормированное пространство и $M \subset X$. Если $M$ ограничено, замкнуто, невыпукло и $X \backslash M$ связно, то существуют открытое полупространство $H$ и множество $\Sigma \subset H \cap M$ такие, что 
i) $\Sigma$ является относительной гранищей некоторого выпуклого множества $u$, следовательно, $\Sigma$ гомеоморфно конечномерной сфере;

ii) $\Sigma$ является ретрактом $H \cap M$.

Вышеприведенный результат о существовании ретракции шара на его пересечение с чебышёвским множеством и лемма 7.1 были использованы в [202] (см. также [72]) для доказательства того, что в конечномерном пространстве со свойством еxt $S^{*}=S^{*}$ всякое ограниченное чебышёвское множество выпукло. Действительно, предположим, что $\overline{\operatorname{ext} S^{*}}=S^{*}$ и существует невыпуклое ограниченное чебышёвское множество $M$. Пусть $H$ и $\Sigma$ выбраны так, как в лемме 7.1 (что возможно, поскольку дополнение к ограниченному чебышёвскому множеству в конечномерном пространстве связно). Полупространство $H$ определяется посредством некоторого функционала $f \in S^{*}$. Слегка изменяя $H$, мы не меняем ситуацию, так что можно предположить, что $f \in \exp S^{*}$. Тогда если гиперплоскость bd $H$ является опорной к некоторому шару, то касание происходит по гладкой точке. Согласно (3.1) можно выбрать замкнутый шар $B_{R}$ достаточно большого радиуса таким образом, что $\Sigma \subset\left(B_{R} \cap M\right) \subset(H \cap M)$. Тогда $\Sigma$ является ретрактом $B_{R} \cap M$, а множество $B_{R} \cap M$ - ретрактом $B_{R}$. Однако это невозможно, так как сфера не может быть ретрактом шара.

Браун [72; теорема 1.6.1] получил следующее обобщение ретракции (7.1) на множества более общего вида, чем чебышёвские. Теорема 7.18 имеет важное следствие - см. теорему 7.19 далее.

ТеОрема 7.18. Пусть $M$ - множество существования в линейном нормированном пространстве $X$. Предположим, что метрическая проекция $P_{M}$ полунепрерывна сверху. Тогда если $M \cap B(x, r) \neq \varnothing$ при некоторых $x \in X$ $u r>0$, то существует полунепрерывная сверху многозначная ретракиия $\Phi$ шара $B(x, r)$ на $M \cap B(x, r)$ такая, что для каждого $z \in B(x, r)$ выполнено равенство $\Phi(z)=P_{M} z^{\prime}$ при некотором $z^{\prime} \in B(x, r)$.

Из теоремы 7.18 вытекает следующий результат (Браун [72; следствие 1.6.2]).

Теорема 7.19. Если ограниченно компактное подмножество линейного нормированного пространства Р-ациклично, то оно В-ациклично.

ЗАмечАниЕ 7.2. Теорема 7.19 может быть сформулирована в чуть более сильном виде: если $M$ является $P$-ацикличным аппроксимативно компактным подмножеством банахова пространства и пересечение $M$ с некоторым шаром $B$ компактно, то $M \cap B$ ациклично.

ЗАмЕчАНИЕ 7.3. Ограничения типа компактности в теореме 7.19 и замечании 7.2 существенны. Известный пример Данхема несвязного чебышёвского множества в $C[0,1]$ (множество в примере Данхема локально компактно, не является солнцем и не аппроксимативно компактно) показывает, что $P$-стягиваемость (в нашем случае - чебышёвость) не влечет $B$-стягиваемость (и даже связность).

Также отметим следующий результат, полученный Брауном [75].

Теорема 7.20. Пусть $X_{n}$ - конечномерное линейное нормированное пространство, $L \subset X$ - подпространство и $M \subset L$ - замкнутое подмножество. Предположим, что $P_{M} x$ ациклично при всех $x$ таких, что для которых $\stackrel{\circ}{B}(x, \rho(x, M)) \cap L \neq \varnothing$. Тогда $M$ является $P$-ацикличным. 
Вышеприведенная теорема была использована Брауном [75] для доказательства того, что солние, лежащее в двумерном подпространстве конечномерного пространства $X_{n}$, является $P$-ацикличным (B-ацикличным), причем приведенное в [75] доказательство технически очень сложно. Однако этот результат Брауна можно установить гораздо проще, если воспользоваться тем, что все солнца в двумерном пространстве монотонно линейно связны [16] (и, как следствие, $P$ - и $B$-ацикличны), и следующим результатом [13].

Для подпространства $H \subset X$ и точки $\theta \in \stackrel{\circ}{B}:=\stackrel{\circ}{B}(0,1)$ мы полагаем:

$|\cdot|_{\theta}-$ несимметричная норма на $X$, задаваемая функционалом Минковского тела $\stackrel{\circ}{B}-\theta$ относительно точки 0 ;

$|\cdot|_{H, \theta}-$ несимметричная норма на $H$, индуцированная несимметричной нормой $|\cdot|_{\theta}$.

Напомним, что несимметричной нормой на $X$ называется неотрицательный сублинейный функционал $\|\cdot\|$ такой, что для всех $x, y \in X$

1) $\|x\|=0$ тогда и только тогда, когда $x=0$;

2) $\|\alpha x\|=\alpha\|x\|$ для всех $\alpha \geqslant 0$;

3) $\|x+y\| \leqslant\|x\|+\|y\|$.

В общем случае $\|x\| \neq\|-x\|$. Отметим, что функция $\|x\|_{\text {sym }}=\max \{\|x\|,\|-x\|\}$, $x \in X$, является нормой. Наиболее полно обзор общей теории несимметрично нормированных пространств приведен в монографии С. Кобзаша [85], охватывающей результаты, полученные до 2013 г.

ТЕОРема 7.21 [13]. Пусть $M$ является чебышёвским множеством, солнцем или строгим солнием в пространстве $(X,\|\cdot\|)$. Тогда:

1) множество $M$ обладает тем же свойством в пространстве $\left(X,|\cdot|_{\theta}\right)$ для любого $\theta \in \stackrel{\circ}{B}$;

2) если $\left(H,|\cdot|_{H, \theta}\right)$ - афбинное подпространство в $X$ и $M \subset H$, то $M$ обладает тем же свойством в пространстве $\left(H,|\cdot|_{H, \theta}\right)$ для любого $\theta \in \stackrel{\circ}{B}$.

Аналогичный результат верен для $\alpha$-солнц и полусолнц.

В п. 2) теоремы 7.21 существенным является условие $M \subset H$. Легко построить примеры, показывающие, что пересечение $M \cap H$, где $M$ - чебышёвское множество в $X, M \not \subset H$, не обязано быть чебышёвским множеством в $H$.

\section{8. Классы солнц и связность}

Классическим является утверждение, что в гладких пространствах (и только в них) всякое солнце (строгое солнце) выпукло (см., например, [194; § 5.3], [132]). Поэтому вопрос о связности солнц и строгих солнц является содержательным только в негладких пространствах. Выше мы видели, что в самом общем случае при наложении на множество ограничений типа связности ( $\stackrel{8}{B}$-бесконечная связность) можно получить весьма сильные результаты о существовании непрерывных $\varepsilon$-выборок и, значит, об устойчивости оператора почти наилучшего приближения. 
8.1. Связность в конечномерных пространствах. Изучение $B$-связности солнц в произвольных пространствах было, что естественно, начато в случае $\operatorname{dim} X=2$. В отличие от случая пространств большей размерности двумерный случай достаточно геометрически нагляден: если $M$ - солнце в двумерном $X_{2}$ и $x \notin M$, то $P_{M} x$ - это точка, отрезок или объединение двух отрезков с концами в одной точке [43] (т. е. в двумерном случае солнца $P$ - и $B$-стягиваемы).

Беренс и Хетцельт [43], [45] для произвольного солнца $M$ в двумерном линейном нормированном пространстве $X_{2}$ установили его метрическую выпуклость относительно радоново трансформированной нормы $\|\cdot\| \#$ (понятие радоново трансформированной нормы введено П. Грубером [117], см. также [43], [16], [25]). Это влечет $P$ - и $B$-стягиваемость (а следовательно, $P$ - и $B$-клеточноподобность и $P$ - и $B$-ацикличность произвольного солнца в $X_{2}$ ). Напомним, что стягиваемость всегда влечет клеточноподобность, которая, в свою очередь, для компактных множеств влечет ацикличность (относительно любой непрерывной теории (ко)гомологий) [120; § с-5]. Для плоских компактов клеточноподобность совпадает с ацикличностью.

Заметим, что ввиду теоремы 7.21 аналогичный результат верен в произвольном линейном нормированном пространстве $X$ для произвольного солнца, содержащегося в двумерном подпространстве пространства $X$.

В конечномерном случае первый общий результат о связности солнц был получен Кощеевым [143; теорема 6] в 1975 г.

ТЕОРема 8.1. В конечномерном линейном нормированном пространстве всякое солние свлзно.

Пытаясь решить задачу о $B$-связности солнц в многомерном случае, Беренс и Хетцельт предъявили первый нетривиальный пример пространства размерности $\geqslant 3$, в котором всякое солнце $B$-связно (и даже $B$-стягиваемо, как следует из теоремы 9.1). Именно, они установили [44] $\ell^{1}$-связность произвольного солнца в $\ell^{\infty}(n)$, что влечет [20] его монотонную линейную связность, $P$ - и $B$-клеточноподобность (а поэтому $P$ - и $B$-ацикличность $[20]$ ). В дальнейшем вопрос о $B$-связности и $B$-ацикличности солнц изучался Брауном [73], [74], [76] и Алимовым [11], [14]--[16].

Наилучший общий результат о связности солнц в произвольном конечномерном пространстве принадлежит Брауну [74; теорема 3].

ТЕОРема 8.2. Если $M$ - солние в конечномерном линейном нормированном пространстве $X$, то оно линейно связно и локально линейно связно.

Более того, существуют положительные константы $L$ u $\alpha$, зависящие только от $X$, такие, что для любых различных точек $x, y \in M$ найдется путь $s:[0,1] \rightarrow M$, соединяющий $x$ u $y$, такой, что

$$
\|s(\xi)-s(\eta)\| \leqslant L\|x-y\| \cdot|\xi-\eta|^{\alpha}
$$

для всех $\xi, \eta \in[0,1]$. 
С учетом теоремы 7.21 из теоремы 8.2 вытекает следующий результат для солнц, содержащихся в конечномерном подпространстве линейного нормированного пространства.

СлЕДСтвиЕ 8.1. Пусть $X$ - линейное нормированное пространство, $M$ солние в $X$, содержащееся в конечномерном подпространстве. Тогда $M$ линейно связно и локально линейно связно.

Существенно более сильные результаты о связности солнц удается получить в так называемых (BM)-пространствах, введенных Брауном [73]. В таких пространствах оказываются верными многие результаты, установленные для солнц в пространстве $\ell^{\infty}(n)$.

ПроБлемА 7. Является ли произвольное строгое солнце в произвольном конечномерном $X_{n}, n \geqslant 4, P$-ацикличным, $P$-клеточноподобным или $P$-стягиваемым?

Ответ неизвестен даже для случая полиэдральных $X_{n}$ (за исключением пространства $\ell^{\infty}(n)$ и, более общо, (BM)-пространств; см. п. 9.3). (В размерности 3 положительный ответ на проблему 7 недавно получен первым автором.)

8.2. Связность в бесконечномерных пространствах. Для пространств бесконечной размерности оказалось, что проблема связности чебышёвских множеств отлична от проблемы связности солнц. В известных примерах несвязных чебышёвских множеств (Данхем [93], Кли [135]) построенные множества не являются солнцами. Единственный пример несвязного солнца построен Кощеевым [146], [147]. Отметим, что построенное Кощеевым несвязное солнце не является чебышёвским множеством.

Укажем, что известно о связности солнц в бесконечномерных линейных нормированных пространствах. Следующий результат принадлежат КощееBy [144].

ТЕОРема 8.3. Ограниченно компактное солнце в линейном нормированном пространстве не имеет собственных ограниченных связных компонент. Как следствие, компактное солнце в произвольном линейном нормированном пространстве связно.

ПроБЛЕмА 8. Верно ли, что ограниченно компактное солнце в бесконечномерном линейном нормированном пространстве связно?

Отметим еще один результат Кощеева [144].

Tеорема 8.4. Пусть $M$ - LG-множество (в частности, $M$ - строгое протосолние). Тогда $M$ не имеет собственных связных компонент, являющихся множествами существования. В частности, $M$ не содержит собственных ограниченно компактных связных компонент.

Кощеев [143; предложение 7] показал, что в локально равномерно выпуклом пространстве всякое солнце $B$-связно. Этот результат усиливает результат Брозовского-Дойча (теорема 5.6) для LUR-пространств, поскольку в силу теоремы 4.3 солнце в LUR-пространстве является чебышёвским солнцем. 
Линейное нормированное пространство называют равномерно неквадратным, если найдется $0<\varepsilon<1$ такое, что $\|(x+y) / 2\| \leqslant 1-\varepsilon$ или $\|(y-x) / 2\| \leqslant 1-\varepsilon$ для всех $x, y \in X,\|x\|=\|y\|=1$. Данное понятие введено Р. С. Джеймсом. Отметим, что нормированное пространство $X$ равномерно неквадратно тогда и только тогда, когда $X^{*}$ равномерно неквадратно [144]. Равномерно неквадратное банахово пространство всегда рефлексивно.

Следующие теоремы 8.5-8.7 принадлежат Кощееву [144].

Теорема 8.5. В равномерно неквадратном банаховом пространстве всякое $\gamma$-солнще (в частности, всякое солнце) связно.

Линейное нормированное пространство называют неквадратным, если его единичная сфера не содержит отрезка длины 2 (что эквивалентно тому, что $S$ не содержит "квадрат").

Теорема 8.6. Для связности каждого $\alpha$-солниа в $X$ необходимо и достаточно, чтобы единичная сфера $S$ была неквадратна.

ТеОрема 8.7. Для связности каждого $\gamma$-солнца в линейном нормированном пространстве $X$ необходимо, чтобы единичная сфера пространства $X^{*}$ бъла неквадратна.

Отметим, что единичная сфера конечномерного пространства $X_{n}$ неквадратна тогда и только тогда, когда $X_{n}$ равномерно неквадратно. Отсюда вытекает [147], что в конечномерном $X_{n}$ всякое $\alpha$-солнце $(\beta-, \delta$-, $\gamma$-солнце) связно тогда и только тогда, когда единичная сфера пространства $X$ неквадратна.

Как следствие, в конечномерном $X_{n}$ всякое $\alpha$-солнце ( $\gamma$-солнце) связно тогда и только тогда, когда $X_{n}$ равномерно неквадратно.

Следующие два результата также получены Кощеевым [147].

Теорема 8.8. Предположим, что $S^{*}$ не содержит отрезков длиной $\geqslant 2-\varepsilon$ при некотором $\varepsilon>0$. Тогда в $X$ любое $\gamma$-солнце связно (u, как следствие, связно любое $\beta$-солнце, $\alpha$-солнце, солние и строгое солнце).

Теорема 8.9. Предположим, что $S^{*}$ не содержит отрезков длиной $\geqslant 2-\varepsilon$ при некотором $\varepsilon>0$. Пусть $M$ есть $\gamma$-солние $и a, b \in M$. Тогда для любой точки $c \in(a, b)$

$$
\rho(c, M) \leqslant(2-\varepsilon)(4-\varepsilon)^{-1}\|(a-b)\| .
$$

Отметим, что ограниченно компактное $L G$-множество в нормированном пространстве $B$-связно [143; предложение 11]. Поскольку по теореме 3.5 строгое протосолнце является LG-множеством, то имеет место следующий результат [143; предложение 12$]$, в котором установлена $B$-связность; $B$-линейная связность обеспечивается (5.1).

ТЕОРема 8.10. Ограниченно компактное строгое солнце в нормированном пространстве В-связно (В-линейно связно, если $X$ банахово).

Как следствие, в банаховом пространстве ограниченно компактное строгое солние (в частности, строгое солние в конечномерном пространстве) линейно связно. Действительно, $B$-связное множество всегда $\stackrel{\circ}{B}$-связно [222], а согласно $(5.1)$ в банаховых пространствах классы замкнутых $\stackrel{\circ}{B}$-связных и замкнутых $\stackrel{\circ}{B}$-линейно связных множеств совпадают. 
Частично обратный результат к теореме 8.10 установлен Кощеевым в [143].

Теорема 8.11. В локально равномерно выпуклом пространстве замкнутое

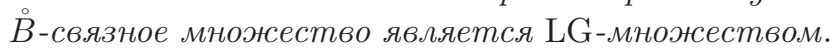

На самом деле строгая выпуклость пространства является необходимым условием для того, чтобы замкнутое $\stackrel{\circ}{B}$-связное множество было LG-множеством. В общем случае связанный с теоремой 8.11 вопрос о характеризации пространств, в которых классы $\stackrel{\circ}{B}$-связных множеств и LG-множеств совпадают, остается открытым.

Следующий результат для случая равномерно выпуклых пространств установлен в [143].

TЕОрема 8.12. В пространстве $X \in(\mathrm{ES})$ каждое замкнутое LG-множество является $\stackrel{\circ}{B}$-связным ( $\stackrel{\circ}{B}$-линейно связным). В пространстве $X \in(\mathrm{ES}) \cap(\mathrm{R})$ класс всех замкнутых $\stackrel{\circ}{B}$-связных $(\stackrel{\circ}{B}$-линейно связных) множеств совпадает с классом замкнутых LG-множеств. В частности, для конечномерного пространства $X_{n}$ условие его строгой выпуклости необходимо и достаточно для того, чтобы класс всех замкнутых $\stackrel{\circ}{B}$-связных (ํㅗ-линейно связных) множеств в $X_{n}$ совпадал с классом замкнутых LG-множеств.

ДокАЗАТЕльство. Следуя [143], предположим, что $M$ является не $\stackrel{\circ}{B}$-связным LG-множеством, т. е. при некоторых $x$ и $r$ пересечение $M \cap \stackrel{\circ}{B}(x, r)$ представляется в виде объединения непустых, замкнутых в $\stackrel{\circ}{B}(x, r)$, непересекающихся множеств $A$ и $B$. Стандартными рассуждениями ситуация сводится к случаю $\rho(x, A)<\rho(x, B)$. Из непрерывности метрической функции $\rho(\cdot, M)$ следует, что существует $\delta>0$ такое, что $\rho(z, A)<\rho(z, B)$ для любого $z \in B(x, \delta)$. В пространстве Ефимова-Стечкина $X$ дополнение к замкнутому непустому множеству содержится в замыкании точек существования для этого множества. Поэтому найдется точка $z \in B(x, \delta)$, у которой $P_{B} z \neq \varnothing$. Соответственно, если $y_{0} \in P_{B} z$, то $y_{0}$ - точка локального минимума, не являющаяся точкой глобального минимума. Противоречие с тем, что $M$ является LG-множеством. Окончательно, $B$-линейная связность обеспечивается равенством (5.1). Обратное утверждение доказывается аналогично теореме 5.2 (см. [203]).

СлЕДСТВИЕ 8.2. В пространстве $X \in(\mathrm{ES}) \cap(\mathrm{R})$ замкнутое $\stackrel{\circ}{B}$-связное множество является луной.

ПроБлЕмА 9. В каких пространствах классы замкнутых LG-множеств и замкнутых $\stackrel{\circ}{B}$-связных множеств совпадают?

Поскольку в равномерно выпуклом пространстве (и даже в пространстве Ефимова-Стечкина, см. теорему 5.9$)$ чебышёвское множество $\stackrel{\circ}{B}$-связно и, более того, $\stackrel{\circ}{B}$-линейно связно, то из теоремы 8.12 вытекает [143], что в равномерно выпуклом банаховом пространстве каждое чебышёвское множество является LG-множеством.

Поскольку строгое протосолнце всегда является LG-множеством, то из теоремы 8.12 и равенства (5.1) вытекает следующий результат (см. [25; следствие 7.2]). 
СледСтвиЕ 8.3. Строгое протосолние в пространстве Ефимова-Стечкина $P_{0}$-компактно и $\stackrel{\circ}{B}$-линейно связно.

Отметим следующий результат [57; теорема 6.1 и следствие 6.3$])$ : если $M-$ строгое солнце в $\ell^{\infty}(n)$, то любые две точки $x, y \in M$ можно соединить монотонной кривой $k(\cdot) \subset M$ (т. е. $k_{i}(t)$ - монотонные функции по $t$ для любого $i=1, \ldots, n)$; при этом, если $x_{i} \neq y_{i}$ для любого $i=1, \ldots, n$, то $k_{i}(t)-$ строго монотонные функции. Отсюда вытекает, что строгое солнце в $\ell^{\infty}(n)$ монотонно линейно связно и, как следствие, $B$-стягиваемо (см. теорему 9.1).

Имеет место следующий результат ([11], см. теорему 9.11 ниже): в пространстве с 0 всякое солнце связно (и, более того, монотонно линейно связно).

8.3. Пример Кощеева несвязного солнца. В отличие от $\alpha$-солнц, которые могут быть несвязны даже на плоскости, для солнц вопрос об их несвязности оказался сложным. Единственный известный пример несвязного солнца в бесконечномерном подпространстве пространства $C[0,1]$ со специально выбранной нормой построен Кощеевым [146]. Напомним построения Кощеева. В $C[0,1]$ рассматривается подпространство

$$
Y=\left\{x \in C[0,1] \mid x(0)=0, \bigvee_{0}^{1}(x)<\infty\right\}
$$

с нормой

$$
\max \left\{\|x\|, 8^{-1} \bigvee_{0}^{1}(x)\right\}
$$

где $\bigvee_{0}^{1}(x)$ - вариация функции $x,\|x\|$ - равномерная норма. Ясно, что $Y-$ банахово пространство. Пусть

$$
\begin{aligned}
& A_{1}:=\left\{x \in C[0,1] \mid x\left(\frac{1}{2 n}\right) \geqslant \frac{1}{2 n}, n=1,2, \ldots\right\}, \\
& B_{1}:=\left\{x \in C[0,1] \mid x\left(\frac{1}{2 n+1}\right) \leqslant-\frac{1}{2 n+1}, n=1,2, \ldots\right\},
\end{aligned}
$$

$A:=A_{1} \cap Y, B:=B_{1} \cap Y, M:=A \cup B$. Множества $A, B$ замкнуты в $Y$, выпуклы, $A \cap B=\varnothing$. В [146] показывается, что для $x \notin M, p_{n}:=1 /(2 n), q_{m}:=1 /(2 n+1)$ выполнены равенства

$$
\begin{aligned}
& \rho(x, A)=\max _{n=1,2, \ldots}\left\{p_{n}-x\left(p_{n}\right)\right\}=p_{n_{0}}-x\left(p_{n_{0}}\right), \\
& \rho(x, B)=\max _{m=1,2, \ldots}\left\{q_{m}+x\left(q_{m}\right)\right\}=q_{m_{0}}+x\left(q_{m_{0}}\right),
\end{aligned}
$$

и строятся классы элементов из $A$ и $B$, на которых достигаются соответственно расстояния $\rho(x, A)$ и $\rho(x, B)$. Следовательно, для $x \notin M$ множества ближайших $P_{A} x$ и $P_{B} x$ непусты. Выбирая $x \notin M$ таким образом, что $\rho(x, A)>\rho(x, B)$, мы находим локальный минимум функции расстояния $\rho(\cdot, M)$, не являющийся глобальным. Таким образом, $M$ не является LG-множеством и, значит, ввиду теоремы 3.5, строгим солнцем. Отметим также, что $M$ не аппроксимативно компактно.

Проблема 10. Верно ли, что существует несвязное строгое солнце, проксиминальное LG-множество, проксиминальная луна? 
8.4. Связность пересечений солнц и чебышёвских множеств с подпространствами и промежутками в пространствах $C(Q)$. В данном пункте мы рассмотрим задачу сохранения (в естественной постановке) аппроксимативных свойств заданного множества $M$ в пространствах типа $C(Q)$ при пересечении с подмножествами пространства. Конечно, чтобы получить положительный результат, множества, с которыми пересекается $M$, должны удовлетворять определенным условиям, и в ряде случаев оказывается возможным полностью охарактеризовать такие множества, при пересечении с которыми сохраняются аппроксимативные свойства исходного множества.

Задача об аппроксимативных свойствах пересечений чебышёвских множеств и различных солнц с подмножествами пространства начала рассматриваться достаточно недавно (см., например, [11], [14]-[16], [19]).

В этом пункте мы рассмотрим данную задачу в конкретных пространствах типа $C(Q)$. Случай общих пространств будет затронут в разделе 9 при изучении монотонно линейно связных и $m$-связных множеств.

Пусть $X=\ell^{\infty}(n)$ или $c_{0}$. Пусть также $k \in \mathbb{Z}_{+}, k \leqslant \operatorname{dim} X$.

Введем следующие обозначения:

$\operatorname{cAff}_{k}(X)$ - класс всех аффинных координатных подпространств из $X$ размерности $k$, т. е. аффинных подпространств вида

$$
\operatorname{lin}\left\{e_{i_{1}}, \ldots, e_{i_{k}} \mid 1 \leqslant i_{1}<\cdots<i_{k} \leqslant n\right\}+x, \quad x \in X
$$

здесь $e_{1}, \ldots, e_{n}-$ стандартный базис в $X=\ell^{\infty}(n)$ или $c_{0}$; элементы из $\mathrm{cAff}_{k}\left(\mathbb{R}^{n}\right)$ называются аффинными координатными подпространствами;

$\mathrm{cAff}^{k}\left(c_{0}\right)$ - класс всех аффинных координатных подпространств коразмерности $k$, т. е. подпространств вида

$$
\left\{x \in c_{0} \mid x_{i_{1}}=c_{1}, \ldots, x_{i_{k}}=c_{k}\right\}
$$

для некоторого фиксированного набора индексов $i_{1}, \ldots, i_{k}$ и набора констант $c_{1}, \ldots, c_{k}$.

Для пространства $\ell^{\infty}(n)$ в данной задаче оказалось возможным получить существенное продвижение [12]. Несмотря на прозрачность утверждений приводимой ниже теоремы 8.13, вопрос об их справедливости долгое время был открыт.

Tеорема 8.13. Пусть $M \subset \mathbb{R}^{n}, 1 \leqslant k \leqslant n-1 u H \in \operatorname{cAff}_{k}\left(\mathbb{R}^{n}\right)-$ координатное афбинное подпространство в $\mathbb{R}^{n}$, норма на котором индуцирована нормой $\|\cdot\|_{\infty}$ в $\mathbb{R}^{n}$. Пусть $M \cap H \neq \varnothing$. Тогда:

а) если $M-$ чебышёвское множество в $\ell^{\infty}(n)$, то $M \cap H-$ чебышёвское множество в $\mathrm{H}$;

b) если $M-$ солнце в $\ell^{\infty}(n)$, то $M \cap H-$ солнце в $H$;

c) если $M$ - строгое солнце в $\ell^{\infty}(n)$, то возможны два варианта: или $M \cap H$ - строгое солнще в $H$, или $M \cap H$ - солнще, но не строгое солнце в $H$; в последнем случае найдется опорный конус $K=\stackrel{\circ}{K}(\xi, \zeta) \subset \mathbb{R}^{n}$ линейной размерности $\geqslant k$ такой, что $H \subset$ bd $K u M \cap \operatorname{int} K=\varnothing$, при этом $M \not \subset H$. 
Напомним, что под линейной размерностью выпуклого множества $C \subset \mathbb{R}^{n}$ понимается размерность максимального аффинного подпространства, содержащегося в $C$. Понятно, что для опорного конуса $\stackrel{K}{K}(x, y)$ в $\ell^{\infty}(n)$ такое максимальное подпространство всегда можно выбрать координатным (экстремальным).

ЗАмЕчАНИЕ 8.1. В п. с) теоремы 8.13 указывается возможность существования такого строгого солнца $M$, сечение которого координатным подпространством $H$ может не быть строгим солнцем в $H$ (а в [9] построен пример такого строгого солнца $M$ и такой гиперплоскости $H$ в $\left.\ell^{\infty}(3)\right)$. Однако такая ситуация не является типичной, так как в таком случае по п. с) гиперплоскость $H$ является опорной к $M$ и, более того, $M \not \subset H$, и поэтому таких "неправильных" гиперплоскостей $H$ не более $2^{n}$.

ЗАмЕчАниЕ 8.2 (о сечениях некоординатными подпространствами). Отметим [14], что если $G$ - гиперплоскость в $\mathbb{R}^{n}$, не являющаяся координатной, то найдется чебышёвское множество $\widehat{M}$ в $\ell^{\infty}(n)$ такое, что сечение $\widehat{M} \cap G$ не будет стягиваемым в $G$ и, следовательно, чебышёвским множеством ни в какой норме на $G$.

Следующий результат [12], имеющий самостоятельный интерес, используется при доказательстве утверждения а) теоремы 8.13.

Теорема 8.14. Пусть $M \subset \mathbb{R}^{n}$ - чебъиёвское множество в $\ell^{\infty}(n)$, и пусть $H \in \mathrm{cAff}_{n-1}\left(\mathbb{R}^{n}\right)$ - координатная аффинная гиперплоскость в $\mathbb{R}^{n}$. Тогда, если $M \cap H \neq \varnothing$, то $M \cap H$ - чебышёвское множество в $(H,\|\cdot\|)$, где норма $\|\cdot\|$ на $H$ индуцирована $\ell^{\infty}$-нормой на $\mathbb{R}^{n}$.

Напомним определение сегмента и промежутка. Следуя [104], [215], для $f_{1}, f_{2}: Q \rightarrow \mathbb{R}$ определим сегмент $\left.\left[f_{1}, f_{2}\right]\right]$ функций:

$$
\llbracket\left[f_{1}, f_{2}\right]=\left\{f \in C(Q) \mid f(t) \in\left[f_{1}(t), f_{2}(t)\right] \forall t \in Q\right\} .
$$

Множество $\varnothing \neq \Pi \subset C(Q)$ называется промежутком, если для всех $f_{1}$, $f_{2} \in \Pi$ выполнено $\left[f_{1}, f_{2}\right] \subset$ П. В [215] установлено, что подмножество $\Pi \subset$ $C(Q)$ является замкнутым промежутком тогда и только тогда, когда П представимо в виде $\Pi=\left[\left[f_{1}, f_{2}\right]\right]$, где $f_{1}, f_{2}: Q \rightarrow \overline{\mathbb{R}}, f_{1} \leqslant f_{2}, f_{1}$ полунепрерывна сверху на $Q$, а $f_{2}-$ снизу.

Рассматривая задачу об аппроксимативных свойствах пересечений чебышёвских множеств и солнц с промежутками, мы вначале отметим следующий достаточно неожиданный результат [14], [10]. (Здесь и ниже ri $A$ обозначает относительную внутренность выпуклого множества $A$.)

Ниже под брусом мы будем понимать брусы пересечения экстремальных гиперполос вида

$$
\{x \in X \mid a \leqslant f(x) \leqslant b\}, \quad-\infty \leqslant a \leqslant b \leqslant+\infty, \quad f \in \operatorname{ext} S^{*},
$$

порождаемых в исходном пространстве экстремальными функционалами из $S^{*}$.

Теорема 8.15. Имеют место следующие утверждения.

1) Чебышёвское множество в $\ell^{\infty}(n)$ является экстремально чебышёвским. Иными словами, если П - брус в $\ell^{\infty}(n)$ и $M$ - чебышёвское множество такое, что $M \cap \Pi \neq \varnothing, M \cap \operatorname{ri} \Pi=\varnothing$, то пересечение $M \cap \Pi$ одноточечно. 
2) Пусть $M$ - аппроксимативно компактное чебышёвское множество в $c_{0}$. Предположим, что П - замкнутьй промежуток конечной коразмерности в $c_{0}, M \cap \Pi \neq \varnothing, M \cap \operatorname{ri} \Pi=\varnothing$. Тогда пересечение $M \cap \Pi$ одноточечно.

В теореме 8.16, которая развивает теорему 8.13, дана характеризация замкнутых множеств $\Pi \subset \mathbb{R}^{n}$, пересечение с которыми чебышёвского множества (солнца, строгого солнца) $M$ в $\ell^{\infty}(n)$ сохраняет (в естественной постановке) аппроксимативные свойства множества $M$. Оказывается, что такие множества П в точности являются замкнутыми промежутками в $\mathbb{R}^{n}$. Полный ответ на аналогичный вопрос для двумерного пространства с произвольной нормой дается в работе [16]. Аналогичный вопрос для монотонно линейно связных множеств и m-связных множеств рассматривается ниже в разделе 9 .

Для $\varnothing \neq M \subset X$ обозначим, как и выше,

$$
T_{M}:=\left\{x \in X \mid \operatorname{card} P_{M} x=1\right\} .
$$

Теорема 8.16. Пусть $\varnothing \neq \Pi \subset \mathbb{R}^{n}$. Имеют место следующие утверждения.

а) Множество П является замкнутым промежутком в $\ell^{\infty}(n)$ тогда и только тогда, когда $\Pi \cap M$ является солнием в $\ell^{\infty}(n)$ для всякого солниа $M$ в $\ell^{\infty}(n), M \cap \Pi \neq \varnothing$.

b) Множество П является замкнутым промежутком в $\ell^{\infty}(n)$ u int $\Pi \neq \varnothing$ тогда и толъко тогда, когда $\Pi \cap M$ является строгим солнием в $\ell^{\infty}(n)$ для любого строгого солниа $M$ в $\ell^{\infty}(n)$ такого, что $M \cap \operatorname{int} \Pi \neq \varnothing$.

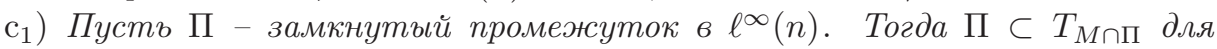
любого чебышёвского множества $M$ в $\ell^{\infty}(n), M \cap \Pi \neq \varnothing$.

$\mathrm{c}_{2}$ ) Пусть множество $\Pi \subset \mathbb{R}^{n}$ связно, замкнуто, и пусть включение $\Pi \subset$

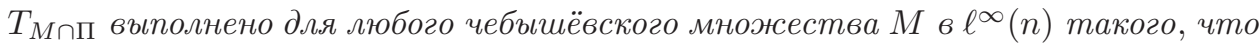
$M \cap \Pi \neq \varnothing$. Тогда $\Pi-$ замкнутый промежуток.

ЗАмечание 8.3. В п. b) теоремы 8.16 условие $M \cap \operatorname{int} \Pi \neq \varnothing$ естественно и его нельзя убрать (в работе [9] построен пример строгого солнца в $\ell^{\infty}(3)$ и координатной гиперплоскости П в $\ell^{\infty}(3)$ такой, что $M \cap П$ не является строгим солнцем в $\ell^{\infty}(3)($ и в $\left.H)\right)$. От требования связности нельзя избавиться и в п. с) достаточно рассмотреть в качестве П "двоеточие".

В бесконечномерном случае имеет место следующий результат [10].

Теорема 8.17. Пусть $M$ - чебъшёвское солнце в $X=c_{0}$, с или $\ell^{\infty}$, и пусть $H$ - координатное подпространство конечной размерности, $M \cap H \neq \varnothing$. Тогда $M \cap H-$-ебышёвское солние в $H$.

\section{5. Количество компонент связности дополнения к чебышёвским} множествам и солнцам. В данном пункте рассматривается вопрос о структурных свойствах дополнения к чебышёвскому множеству или солнцу. В основном исследуется конечномерный случай и рассматривается задача о количестве компонент связности дополнения к множеству. При этом стоит подчеркнуть, что здесь мы не рассматриваем случай чебышёвских или протосолнечных каверн в бесконечномерном случае. Стоит отметить, что данный вопрос оказывается связанным с задачами комбинаторной геометрии выпуклых множеств. 
Структура дополнения к чебышёвским множествам и солнцам и, в частности, задача о числе компонент связности множества изучались в [3], [4], [7], [8]. Легко проверить, что дополнение к ограниченному солнцу всегда связно (ср. $[72 ; \S 1.4 .2])$. В конечномерном пространстве всякое чебышёвское множество является солнцем и, следовательно, дополнение к ограниченному чебышёвскому множеству связно.

Для симметрично или несимметрично нормированного пространства $X$ напомним, что точки $s_{1}, \ldots, s_{n} \in S$ называются попарно далекими [3] (или образующими антиподальное семейство), если для тел $B_{i}=B-s_{i}, i=1, \ldots, n$, выполнены условия

$$
B_{i} \cap \operatorname{int} B_{j}=\varnothing, \quad i, j=1, \ldots, n,
$$

т. е. сдвиги $B_{i}$ тела $B$ не перекрываются внутренностями и имеют общую точку 0. В симметричном случае условие (8.2) эквивалентно тому, что $\left\|s_{i}-s_{j}\right\|=2$, $i \neq j$.

Введем следующие обозначения:

(i) $k(B)$ - мощность максимального антиподального семейства шара $B$ (максимальное число неперекрывающихся внутренностями сдвигов шара $B$, имеющих общую точку); в конечномерном $X$ число $k(B)$ всегда конечно;

(ii) $k_{\exp }(B)$ - мощность максимального антиподального семейства шара $B$, состоящего из достижимых точек.

Достаточно очевидно, что если $\operatorname{dim} X=n<\infty$, то

$$
2 \leqslant k_{\exp }(B) \leqslant k(B) \leqslant 2^{\operatorname{dim} X},
$$

причем оценка сверху в последнем неравенстве достигается в том и только том случае, если $B$ есть $n$-мерный параллелепипед [3]. Например, если $B-$ единичный шар пространства $\mathbb{R}^{n}$ с обычной евклидовой нормой, то

$$
k(B)=k_{\exp }(B)=2
$$

если $B$ - аффинный правильный шестиугольник или треугольник на несимметрично нормированной плоскости, то

$$
k(B)=k_{\exp }(B)=3 ;
$$

если же $B \subset \mathbb{R}^{3}$ - “игральная кость" (т. е. трехмерный куб со сглаженными вершинами), то

$$
k(B)=4,
$$

в то время как $k_{\exp }(B)=2$.

В задаче о числе компонент связности дополнений чебышёвских множеств и солнц получены следующие результаты. Теорема 8.18 установлена в [3], теорема 8.19 содержится в [7] и [8], теорема 8.20 доказана в [4], [7].

Характеризация пространств (нормированных или несимметрично нормированных, не обязательно конечномерных), содержащих солнце с заданным количеством компонент связности в дополнении, дается следующей теоремой [3]. 
Теорема 8.18. Пусть $X$ - линейное несимметрично нормированное пространство, $\nu$ - кардинальное число. Для того чтобы в Х существовало солнце

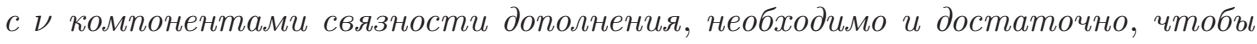
выполнялосъ неравенство $k(B) \geqslant \nu$.

Следующая теорема дает ответ на вопрос о числе компонент связности дополнения к строгому солнцу в конечномерном пространстве.

ТЕОРема 8.19. Пусть $X$ - линейное несимметрично нормированное пространство, $\operatorname{dim} X<\infty$, и пусть $\nu$ - натуральное число. B пространстве $X$ существует строгое солние с $\nu$ компонентами связности в дополнении тогда и только тогда, когда $k(B) \geqslant \nu$.

Теорема 8.20. Пусть $X$ - конечномерное линейное несимметрично нормированное пространство. Пусть $\nu \in\left\{1, \ldots, k_{\exp }(B)\right\}$. Тогда в $X$ найдется чебышёвское множество с $\nu$ компонентами связности дополнения.

Аналогичный вопрос о количестве компонент дополнения чебышёвского множества более труден и связан с классическим вопросом о характеризации конечномерных пространств, в которых всякое чебышёвское множество выпукло. Полный ответ получен только в размерностях $\leqslant 4$ (см. [8]).

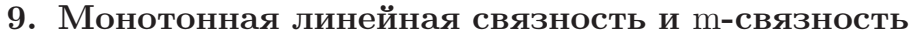

В данном разделе рассматриваются два вида связности множеств: т-связность (связность по Менгеру) и монотонная линейная связность, являющаяся усилением понятия линейной связности, - в отличие от линейной связности для монотонно линейного связного множества предполагается, что любые две его точки можно связать монотонной непрерывной кривой.

9.1. Свойства монотонно связных и m-связных множеств. Следуя Брауну [73], для ограниченного множества $\varnothing \neq M \subset X$ определим $\mathrm{m}(M)$ - оболочку Банаха-Мазура множества $M$, т. е. пересечение всех замкнутых шаров, содержащих $M$. Подмножество $M \subset X$ называется т-связным (связным по Менгеру) (см. [73]), если $\mathrm{m}(\{x, y\}) \cap M \neq\{x, y\}$ для любых различных точек $x, y \in M$. Для краткости далее обозначаем $\mathrm{m}(\{x, y\})=\mathrm{m}(x, y)$.

$\mathrm{K}$ примеру, в пространстве $C(Q)$ структура $\mathrm{m}(M)$ вполне ясна (см., например, [73; теорема 3.1]):

$$
\mathrm{m}(x, y)=\{z \mid z(q) \in[x(q), y(q)], q \in Q\}=: \llbracket[x, y] .
$$

Аналогичное представление также верно и в пространстве $C_{0}(Q)(Q$ - локально компактное хаусдорфово пространство); см. [67].

Для дальнейшего напомним определение сегмента и промежутка в произвольном линейном нормированном пространстве.

По аналогии с (8.1) определим сегмент в произвольном линейном нормированном пространстве $X$ формулой

$$
\left[[x, y]:=\left\{z \in X \mid \min \{\varphi(x), \varphi(y)\} \leqslant \varphi(z) \leqslant \max \{\varphi(x), \varphi(y)\} \forall \varphi \in \operatorname{ext} S^{*}\right\} ;\right.
$$


здесь ext $S^{*}$ - множество экстремальных (крайних) точек единичной сферы $S^{*}$ сопряженного пространства $X^{*}$ (множество экстремальных точек всегда непусто). Аналогия объясняется тем, что каждый экстремальный функционал $f \in C(Q)^{*}$ (или $f \in C_{0}(Q)^{*}$ ) имеет вид $f(x)= \pm x(t)$, где $x \in C(Q)$ или $x \in C_{0}(Q), t \in Q$.

Имея в своем распоряжении понятие сегмента, мы можем по аналогии с вышесказанным определить понятие промежутка в линейном нормированном пространстве $X$ : множество $\varnothing \neq \Pi \subset X$ называется промежутком, если

$$
[[x, y] \subset \Pi \quad \text { для всех } x, y \in \Pi .
$$

Любой замкнутый шар является замкнутым промежутком. Действительно, пусть $u, v \in B$. Если при каком-либо $w \in B$ имеем $f(w) \in[f(u), f(v)]$ для любого $f \in \operatorname{ext} S^{*}$, то по теореме Крейна-Мильмана это включение распространяется на все $f \in S^{*}$, откуда следует, что $\|w\| \leqslant 1$, т. е. $w \in B$.

Отметим, что функциональные промежутки естественно возникают в выпуклом и нелинейном анализе, в задачах теории приближений и оптимального управления, в задаче о расстоянии до чебышёвского подпространства, в теоремах о сильной единственности экстремальных элементов в данной задаче [133], а также в задаче оценки поперечников функциональных классов при пересечении с промежутками [216].

Следуя Франкетти и Роверси [105], введем в рассмотрение класс (MeI) пространств $X$ :

$$
\mathrm{m}(x, y)=\llbracket[x, y] \quad \text { для всех } x, y \in X
$$

(сокращение (MeI) происходит от английского "The hull $\mathrm{m}(x, y)$ equals the interval $\left[[x, y]\right.$ for all $\left.x, y^{\prime \prime}\right)$.

Сразу заметим, что включение

$$
\mathrm{m}(x, y) \supset \llbracket[x, y]
$$

имеет место в любом $X$ (см., например, [105; теорема 3.1]). Действительно [115; с. 55] (см. также [176]), замкнутое выпуклое множество $M$ является пересечением шаров тогда и только тогда, когда для любой точки вне $M$ найдется замкнутый шар, содержащий $M$, но не содержащий эту точку. Теперь осталось вспомнить понятный результат (вытекающий из того, что $\|x\|=\sup _{f \in \operatorname{ext} S^{*}} f(x)$ ) о том, что точку, не принадлежащую замкнутому шару, всегда можно строго отделить от шара посредством экстремальной гиперплоскости.

ЗАмечание 9.1. Равенство

$$
\mathrm{m}(x, y)=\llbracket[x, y]
$$

имеет место для достаточно широкого класса банаховых пространств. Франкетти и Роверси [105; теорема 3.2] показали, что (9.3) имеет место для пространств, на единичной сфере которых точки гладкости образуют всюду плотное множество (в сильной топологии). K таким пространствам относятся слабо асплундовы пространства (в частности, слабо компактно порожденные пространства, а значит, сепарабельные пространства и рефлексивные пространства). Также отметим, что если пространство $X$ таково, что $\operatorname{ext} S^{*}$ лежит 
в замыкании множества $w^{*}$-полуострых точек шара $B^{*}$ (условие Морено), то $\llbracket[x, y]=\mathrm{m}(x, y)$ для всех $x, y \in X$; такому условию, в частности, удовлетворяют конечномерные пространства и пространства со свойством пересечения Мазура [20]. Напомним, что точка $f \in S^{*}$ называется $w^{*}$-полуострой точкой сопряженного шара $B^{*}$ (см., например, [110]), если для любого $\varepsilon>0$ найдется $w^{*}$-срез $S \ell$ шара $B^{*}$ такой, что $\operatorname{diam}(\{f\} \cup S \ell)<\varepsilon$. Здесь $S \ell\left(B^{*}, x, \delta\right):=\{g \in$ $\left.S^{*} \mid g(x)>1-\delta\right\}, 0<\delta<1, x \in X$.

Отметим, что условие Морено не выполнено для пространства $\ell^{1}$ (на единичном шаре пространства $\ell^{\infty}$ отсутствуют $w^{*}$-полуострые точки [167]), но согласно указанной теореме Франкетти-Роверси в $\ell^{1}$ равенство (9.3) имеет место.

В конечномерном случае равенство $[[x, y]=\mathrm{m}(x, y)$ установлено Брауном [73].

Пусть $k(\tau), 0 \leqslant \tau \leqslant 1,-$ непрерывная кривая в линейном нормированном пространстве $X$. Следуя [67], говорим, что кривая $k(\cdot)$ монотонна, если $f(k(\tau))$ является монотонной функцией по $\tau$ для любого $f \in \operatorname{ext} S^{*}$.

Замкнутое подмножество $M \subset X$ называется монотонно линейно связнъмм [15], если любые две точки из $M$ можно соединить непрерывной монотонной кривой (дугой) $k(\cdot) \subset M$. Отметим, что монотонно линейно связное множество всегда $B$-связно (т. е. его пересечение с любым замкнутым - а следовательно, и с открытым [222] - шаром связно; ср. [67; предложение 1.3]).

Сразу отметим, что существуют примеры конечномерных пространств, в которых имеются не т-связные (и, a fortiori, не монотонно линейно связные) чебышёвские множества и не т-связные солнца (см. пример 9.1 в п. 9.2 ниже).

ЗАмЕЧАНИЕ 9.2. Используя (9.2), несложно проверить, что монотонно линейно связное множество с необходимостъю т-связно. Обратное утверждение неверно даже для замкнутых множеств - соответствующий пример в $C[0,1]$ предложен Франкетти и Роверси [105]: пусть

$$
M=M_{1} \cup M_{-1},
$$

где $M_{\sigma}=\{x \in C[0,1] \mid x(0)=\sigma\}, \sigma= \pm 1$. Тогда $M$ состоит из двух выпуклых непересекающихся компонент, в то же время несложно проверить, что $M$ является m-связным.

Однако в $c_{0}$ и в произвольном конечномерном пространстве $X_{n}$ эти свойства эквивалентны для замкнутых множеств (см. теорему 1 в [11] и лемму 9.2); утверждение для $X_{n}$ следует из (9.2), (9.3) и теоремы 9.1 ниже).

Некоторые достаточные условия монотонной линейной связности m-связного подмножества линейного нормированного пространства даны в теореме 9.1. Отметим, что пространствах $X_{n}$ со свойством $\overline{\operatorname{ext} S^{*}}=S^{*}$ класс монотонно линейно связных (m-связных) замкнутых множеств совпадает с классом замкнутых выпуклых множеств (в таких $X$ по теореме Фелпса [176] всегда выполнено равенство $\mathrm{m}(x, y)=[\llbracket x, y]$ для любых $x, y \in X)$.

Нам также понадобится следующий класс пространств, введенный Франкетти и Роверси [105]: 
При этом в определении класса (Ex- $\left.w^{*} \mathrm{~s}\right)$ мы всегда предполагаем, что

$$
\begin{gathered}
F=\left(f_{i}\right)_{i \in I} \subset \operatorname{ext} S^{*} \text { является } w^{*} \text {-плотным в } \operatorname{ext} S^{*}, \\
\operatorname{card} I \leqslant \aleph_{0}, \quad F=-F
\end{gathered}
$$

(сокращение $\left(\mathrm{Ex}-w^{*} \mathrm{~s}\right)$ происходит от немецкого "Die Extrempunktmenge der konjugierten Einheitskugel ist $w^{*}$-separabel").

Сразу отметим, что любое пространство из класса (Ex- $\left.w^{*} \mathrm{~s}\right)$ имеет $w^{*}$-сепарабельный единичный шар. Действительно, так как единичный шар $B^{*}$ пространства $X^{*}$ является $w^{*}$-замыканием выпуклой оболочки множества $\operatorname{ext} S^{*}$, то $w^{*}$-сепарабельность множества ext $B^{*}$ (границы Джеймса) влечет $w^{*}$-сепарабельность шара $B^{*}$ и, как следствие [86; с. 253], $w^{*}$-сепарабельность пространства $X^{*}$. В [86; утверждение $\left.(2)\right]$ показано, что $w^{*}$-сепарабельность шара $B^{*}$ эквивалентна тому, что пространство $X$ изометрически изоморфно подпространству пространства $\ell^{\infty}$ (известно, что последнее выполнено для всех сепарабельных банаховых пространств). Отметим, что в [86] также предъявлен пример банахова пространства такого, что $X^{*} w^{*}$-сепарабельно, а шар $B^{*}-$ нет.

Далее, хорошо известно [20], что если $X$ - сепарабельное линейное нормированное пространство, то $w^{*}$-топология единичного шара $B^{*}$ сопряженного пространства $X^{*}$ метризуема. Отсюда следует, что любое сепарабелъное пространство лежит в классе (Ex- $\left.w^{*} \mathrm{~s}\right)$. Также отметим, что класс $\left(\mathrm{Ex}-w^{*} \mathrm{~s}\right)$ содержит несепарабельное пространство $\ell^{\infty}$ (как пространство непрерывных функций на стоун-чеховской компактификации натурального ряда). При этом $C(Q)$ на несепарабельном (неметризуемом) $Q$ и $c_{0}(\Gamma)$ на несчетном $\Gamma$ не лежат в $\left(\mathrm{Ex}-w^{*} \mathrm{~s}\right)$.

Отметим следующий вопрос, поставленный О. Нигаардом.

Проблема 11. Верно ли, что граница Джеймса банахова пространства $X$ $w^{*}$-сепарабельна в случае, если шар $B_{X *}$ является $w^{*}$-сепарабельным?

Кратко суммируя сказанное выше относительно пространств классов (MeI) и $\left(\mathrm{Ex}-w^{*} \mathrm{~s}\right)$, отметим, что

класс $(\mathrm{MeI}) \cap\left(\mathrm{Ex}-w^{*} \mathrm{~s}\right)$ содержит все сепарабельные банаховы пространства (в частности, все пространства $C(Q)$ на метризуемом компакте $Q$ ) и несепарабельное пространство $\ell^{\infty}$.

Пусть пространство $X$ принадлежит классу $\left(\mathrm{Ex}-w^{*} \mathrm{~s}\right)$, а $F=\left(f_{i}\right)_{i \in I}$ - семейство функционалов из определения класса $\left(\mathrm{Ex}-w^{*} \mathrm{~s}\right)$ (мы всегда предполагаем, что $F=-F)$; пусть также $\left(\alpha_{i}\right) \subset \mathbb{R}, \alpha_{i}>0, i \in I$, $\operatorname{card} I \leqslant \aleph_{0}$, и $\sum \alpha_{i}<\infty$. Для $x \in X$ положим

$$
|x|=\sum_{i \in I} \alpha_{i}\left|f_{i}(x)\right| .
$$

Тогда $|\cdot|$ - норма на $X$, которую, следуя Брауну [73], мы называем ассоциированной (по Брауну). Ясно, что $|x| \leqslant\|x\| \sum \alpha_{i}$.

Важность ассоциированной нормы показывает следующий результат [20], который является естественным обобщением следствия 3.2 из [73], доказанного Брауном в случае $\operatorname{dim} X<\infty$. 
Лемма 9.1. Пусть $X$ - банахово пространство из класса $(\mathrm{MeI}) \cap\left(\mathrm{Ex}-w^{*} \mathrm{~s}\right)$ (в частности, $X$ - сепарабельное банахово пространство), и пусть $x, y \in X$. Следуюшие условия эквивалентны:

a) $z \in \mathrm{m}(x, y)$;

b) для всех $i \in I$

$$
\left|f_{i}(x)-f_{i}(y)\right|=\left|f_{i}(x)-f_{i}(z)\right|+\left|f_{i}(z)-f_{i}(y)\right|,
$$

где $F=\left(f_{i}\right)_{i \in I}$ - семейство из определения класса $\left(\mathrm{Ex}-w^{*} \mathrm{~s}\right)$;

c) $|x-y|=|x-z|+|z-y|$ (m.е. $z$ находится между $x$ и у относительно нормы $|\cdot|)$.

Основным результатом о связи монотонно линейно связных и m-связных множеств является следующая теорема [20].

Теорема 9.1. Пусть $X$ - банахово пространство, и пусть множество $M \subset X$ - замкнуто и т-связно. Предположим, что выполнено хотя бы одно из следующих условий:

а) $M$ ограниченно компактно (в норме $\|\cdot\|)$;

b) $M$ является $|\cdot|$-замкнутым, a $\mathrm{m}(x, y)$ является|-|-компактным для любых $x, y \in X$

с) $\mathrm{m}(x, y)$ является $\|\cdot\|$-компактным для любих $x, y \in X$.

Тогда $M$ - монотонно линейно связно.

Если вдобавок множество $M$ ограниченно компактно, то оно $P$ - и $B$-клеточноподобно, $P$ - и В-ациклично (относительно любой непрерывной теории (ко)гомологий) и является солнием.

Если $X$ конечномерно, то $M$ является $P$ - и B-стягиваемым.

С учетом того, что замкнутое множество $M \subset X$ обладает непрерывной мультипликативной (аддитивной) $\varepsilon$-выборкой для всех $\varepsilon>0$ тогда и только тогда, когда оно является $\stackrel{\circ}{B}$-бесконечно связным (теорема 7.6 ), из теоремы 9.1 вытекает, что m-связное (монотонно линейно связное) замкнутое подмножество конечномерного пространства обладает непрерывной мультипликативной (аддитивной $) \varepsilon$-выборкой для всех $\varepsilon>0$. Более общий результат для аппроксимативно компактных множеств дается теоремой 7.9.

Для случая слабо компактных множеств мы имеем следующий результат.

Теорема 9.2. Пусть $X$ - сепарабельное банахово пространство, и пусть

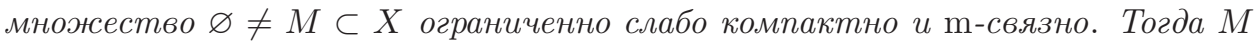
монотонно линейно связно.

ЗАмЕчАниЕ 9.3. Хорошо известен следующий результат Власова [222]: в банаховом пространстве Р-ацикличное ограниченно компактное множество является солнием (см. замечание 7.1 по поводу определения ацикличности, использованного при доказательстве теоремы Власова). Было бы интересно, особенно в свете теоремы 9.2, распространить теорему Власова на случай ограниченно слабо компактных множеств хотя бы в сепарабельном случае. Проблема здесь в следующем: несмотря на то что слабо компактное подмножество $M$ сепарабельного банахова пространства метризуемо, а топология, порождаемая метрикой на $M$, совпадает со слабой топологией на $M$, теорема 
Эйленберга-Монтгомери о неподвижной точке не применима, поскольку метрическая проекция на $M$ полунепрерывна сверху лишь сильно-слабо (носитель один, а топологии на нем разные).

Отметим еще один результат, вытекающий из теоремы 9.1 и теоремы Власова (см. замечание 9.3).

ТЕОРема 9.3. Монотонно линейно связное ограниченно компактное подмножество банахова пространства является солнцем.

Напомним еще одну известную проблему, которая до настоящего времени полностью не решена.

ПроБЛЕма 12. Верно ли, что слабо компактное чебышёвское множество в банаховом пространстве является солнцем?

Первое утверждение теоремs 9.1 идейно восходит к одному конечномерному результату Браесса [57; §6] и следующей лемме Менгера [166].

Напомним, что множество $M$ в метрическом пространстве $(X, d)$ называется метрически выпуклым (или выпуклым по Менгеру), если для любых $x, y \in M$ найдется $z \in M, z \neq x, y$, такое, что $d(x, y)=d(x, z)+d(z, y)$.

Лемма 9.2. Пусть $M$ - полное метрически выпуклое метрическое пространство. Тогда для любых точек $x, y$ из $M$ существует соединяющий их метрический сегмент, т.е. существует изометрия $\varphi:[0, \rho(x, y)] \rightarrow M$ такая, что $\varphi(0)=x, \varphi(\rho(x, y))=y$.

Утверждение теоремы 9.1 о монотонной линейной связности установлено в [15]. Конечномерный случай вытекает из условия а) и теоремы Брауна [74], согласно которой пересечение m-связного замкнутого подмножества конечномерного пространства $X$ с замкнутым шаром бесконечно связно. Отсюда и из известной характеризации абсолютных ретрактов (см., например, [122; теорема 11.1]) следует, что $M$ стягиваемо и локально стягиваемо. Отметим, что условия b) и с) теоремы 9.1 a fortiori выполнены в пространстве $X=c_{0}$.

Отметим, что теорема 9.1 усиливает следующий конечномерный результат Брауна [74].

Теорема 9.4. Пусть $M$ - замкнутое т-связное подмножество конечномерного линейного нормированного пространства. Тогда $M$ бесконечно связно и его пересечение с любым замкнутым шаром также бесконечно связно.

9.2. Солнечность и монотонная линейная связность. Долгое время результаты Кощеева (см. пп. 8.1 и 8.2) о связности компактных солнц в произвольных линейных нормированных пространствах не удавалось улучшить, даже в конкретных конечномерных пространствах. Существенные продвижения в этом направлении получены Беренсом и Хетцельтом [43] и [44], которые установили метрическую выпуклость солнц в произвольных двумерных пространствах и в пространстве $\ell^{\infty}(n)$ (см. также [45], [117]). Как следствие, солнца в таких пространствах монотонно линейно связны.

Сразу отметим, что существуют примеры конечномерных пространств, в которых имеются не т-связные (не монотонно линейно связные) чебышёвские солнца (см. [73; теорема 4.3], [105; с. 19]). Приведем еще один пример. 
Пример 9.1. Пусть $X$ - линейное нормированное пространство, $\operatorname{dim} X<\infty$, со свойством $\overline{\operatorname{ext} S^{*}}=S^{*}$. Фелпс [176] показал, что свойство $\overline{\operatorname{ext} S^{*}}=S^{*}$ выполнено для пространства $X_{n}$ тогда и только тогда, когда каждое выпуклое, ограниченное, замкнутое подмножество $X_{n}$ представимо как пересечение замкнутых шаров (иными словами, такое $X_{n}$ принадлежит классу (MIP), т. е. удовлетворяет свойству пересечения Мазура); как следствие, в таком пространстве монотонная линейная связность замкнутого множества равносильна его выпуклости. Далее, для любого $n \geqslant 3$ Царьков построил пример пространства $X$ размерности $n$ со свойством $\overline{\operatorname{ext} S^{*}}=S^{*}$, содержащего неограниченное невыпуклое чебышёвское множество $M^{\prime}$; при этом любое ограниченное чебышёвское множество в $X$ выпукло. Таким образом, $M^{\prime}$ служит примером $B$-ацикличного $(P$-ацикличного) множества (чебышёвского солнца), не являющегося монотонно линейно связным.

Следующий вопрос поставлен П. А. Бородиным в связи с примером 9.1.

Проблема 13. Верно ли, что ограниченное чебышёвское множество монотонно линейно связно?

Несмотря на то что в настоящей работе вопрос о солнечности чебышёвских множеств практически не затрагивается, стоит упомянуть следующий результат [17], в котором солнечность произвольного чебышёвского множества в линейном нормированном пространстве устанавливается при наложении структурных ограничений типа связности.

Теорема 9.5. Пусть $M$ - монотонно линейно связное подмножество линейного нормированного пространства. Предположим, что $P_{M} x=\{y\} \partial л я$ некоторого $x \notin M$. Тогда $x$ - точка солнечности (y- точка светимости).

Как следствие, монотонно линейно связное чебышёвское множество в линейном нормированном пространстве является солнцем.

Теорему 9.5 можно рассматривать как первый результат, в котором солнечность чебышёвского множества устанавливается при наложении на него структурных ограничений типа связности.

ПроблемА 14. Охарактеризовать конечномерные (бесконечномерные) пространства (размерности $\geqslant 3)$, в которых $B$-ацикличные ( $P$-ацикличные) ограниченно компактные множества являются монотонно линейно связными (ср. пример 9.1).

Выше мы отмечали, что ограниченно компактное монотонно линейно связное множество является солнцем (теорема 9.1). Из теорем 7.15 и 7.9 вытекает следующий результат.

TеОрема 9.6. Аппроксимативно компактное монотонно линейно связное подмножество банахова пространства является $\delta$-солнцем.

9.3. (ВМ)-пространства и их свойства. При изучении связности солнц в конечномерных Браун [73] ввел класс (BM) линейных нормированных пространств. На такие пространства оказывается возможным перенести со случая $X=\ell^{\infty}(n)$ ряд нетривиальных результатов о геометрическо-топологических 
свойствах солнц. Напомним, что линейное нормированное пространство $X$ называется (ВМ)-пространством, если

$$
B(0,\|x\|) \cap(\mathrm{m}(x, y) \backslash\{x\}) \neq \varnothing, \quad \text { когда } \quad[x, x-y] \cap \stackrel{\circ}{B}(0,\|x\|)=\varnothing .
$$

Класс (BM)-пространств содержит в себе все гладкие пространства, все двумерные пространства с полигональным единичным шаром, пространства $\ell^{\infty}(n)$, $c_{0}, c, \ell^{\infty}$, все замкнутые идеалы пространства $C(Q)$, все подрешетки $C(Q)$ с единицей [105]; этот класс замкнут по отношению к формированию конечной $\ell^{\infty}$-прямой суммы [73; раздел 5] и бесконечной $c_{0}$-прямой суммы сепарабельных (BM)-пространств [105; теорема 8.7]. (Если $X_{1}, X_{2}$ - линейные нормированные пространства, то $\ell^{\infty}$-прямой суммой $X_{1}$ и $X_{2}$ называется прямая сумма $X_{1}$ и $X_{2}$ с нормой $\left\|\left(x_{1}, x_{2}\right)\right\|_{\infty}=\max \left\{\left\|x_{1}\right\|_{X_{1}},\left\|x_{2}\right\|_{X_{2}}\right\}$.) Строго выпуклое пространство лежит в классе (BM) тогда и только тогда, когда оно гладкое [105; предложение 8.2]. По поводу следующего результата см. [25; § 8.3].

ПрЕДЛОЖЕНИЕ 9.1. Пространства $\ell^{1}, \ell^{1}(n)$ не принадлежат (BM), $n \geqslant 3$.

Браун [76], [73] (см. теорему 9.7 ниже) установил, что полиэдральные (BM)пространства конечной размерности являются $\ell^{\infty}$-прямыми суммами

$$
X=X_{1} \oplus_{\infty} \cdots \oplus_{\infty} X_{r}
$$

конечного набора симметричных полиэдральных пространств $X_{1}, \ldots, X_{r}$ размерности 1 или 2. Шар пространства вида (9.6) является зонотопом [76] (проекцией $n$-мерного куба на подпространство), но класс всех зонотопов не исчерпывается такими пространствами. Браун [75] получил характеризацию трехмерных (BM)-пространств: $X$ гладко или имеет вид $X=Y \oplus_{\infty} \mathbb{R}$, где $Y$ двумерное (BM)-пространство.

Пусть $n, k \in \mathbb{N}, n>k \geqslant 2$. Мы говорим, что пространство $X$ имеет $n . k$-свойство пересечения (и пишем $X \in(n . k$.I.P.)), если для любого набора из $n$ замкнутых шаров $B\left(a_{i}, r_{i}\right), i=1, \ldots, n$, таких, что $\bigcap_{r=1}^{k} B\left(a_{i_{r}}, i_{r}\right) \neq \varnothing$ при

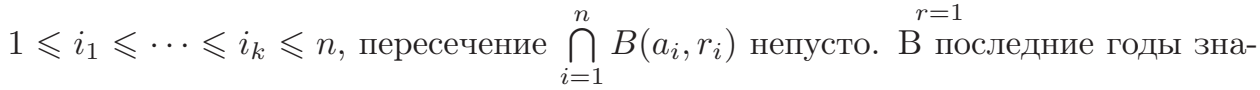
чительный интерес в изучении (n.k.I.P.)-пространств связан с вопросами минимального заполнения подмножеств банаховых пространств и оптимальными сетями (см., например, [36]).

Далее, выпуклое ограниченное замкнутое множество $M$ называется множеством Мазура [116], если для любой гиперплоскости $H$, находящейся на положительном расстоянии от $M$, найдется шар $B^{\prime}$ такой, что $M \subset B^{\prime}$ и $H \cap B^{\prime}=\varnothing$. Пространства, в которых класс множеств Мазура совпадает с классом пересечений замкнутых шаров, называются пространствами Мазура.

Отметим следующий результат [21].

ПреДлОжЕНИЕ 9.2. Следующие утверждения эквивалентны в классе конечномерных полиэдралъных пространств $X$ :
a) $X \in(\mathrm{BM})$;
b) $X$ - пространство Мазура;
c) $X \in$ (4.3.I.P.). 


\section{4. Монотонная линейная связность и $\mathrm{m}-$-вязность солнц в конеч-} номерных (BM)-пространствах. Для формулировки основных результатов этого пункта нам потребуется следующее определение.

Пусть $X$ - конечномерное линейное нормированное пространство, $\operatorname{dim} X=n$. Подпространство $L \subset X$ называется $v$-подпространством (от англ. vertex subspace) [76], если $L$ есть линейная комбинация экстремальных точек единичной сферы $S$, содержащихся в $L$. Следуя Брауну, мы говорим, что отрезок $\left[s_{1}, s_{2}\right] \subset S$ пересекается с подпространством $L$, если $\left[s_{1}, s_{2}\right] \cap L \neq \varnothing$ и $s_{1}, s_{2} \notin L$.

Если $v_{1}, \ldots, v_{r}$ - линейно независимые точки из $\operatorname{ext} S$ и отрезок $\left[s_{1}, s_{2}\right]$ пересекается с подпространством $\operatorname{lin}\left\{v_{1}, \ldots, v_{r}\right\}$, то точки $s_{1}, v_{1}, \ldots, v_{r}$ также линейно независимы и можно найти точки $v_{r+1}, \ldots, v_{n-1} \in \operatorname{ext} S$ такие, что $s_{1}, v_{1}, \ldots, v_{n-1}$ образует базис $X$. В этом случае отрезок $\left[s_{1}, s_{2}\right]$ пересекает гиперподпространство $\operatorname{lin}\left\{v_{1}, \ldots, v_{n-1}\right\}$. Таким образом, отрезок $\left[s_{1}, s_{2}\right]$ пересекается с некоторым $v$-подпространством в $X$ тогда и только тогда, когда он пересекается с некоторым $v$-гиперподпространством.

В следующей теореме все эквивалентности получены Брауном [76], [73], за исключением эквивалентностей со свойством b). Эквивалентность b) $\Leftrightarrow$ a) обеспечивается теоремой 9.1 .

ТЕОРЕма 9.7. В полиэдральном конечномерном линейном нормированном пространстве $X$ следующие условия эквивалентнь:

а) каждое солнце в $X$ является т-связным;

b) каждое солнце является монотонно линейно связным;

c) пространство $X^{*}$ обладает следующим свойством: никакая одномерная грань сферы $S^{*}$ не пересекается ни с каким $v$-подпространством в $X^{*}$;

d) $X^{*}$ есть $\ell^{1}$-прямая сумма вида

$$
X^{*}=Y_{1} \oplus_{1} \cdots \oplus_{1} Y_{r},
$$

где $\operatorname{dim} Y_{i}=1$ или $2, i=1, \ldots, r$;

е) $X$ есть $\ell^{\infty}$-прямая сумма вида

$$
X=X_{1} \oplus_{\infty} \cdots \oplus_{\infty} X_{r},
$$

где $\operatorname{dim} X_{i}=1$ или $2, i=1, \ldots, r$;

f) $X$ является (BM)-пространством.

Если $\mathrm{Q}$ - некоторое свойство, то мы будем говорить, что множество $M$ экстремально $\mathrm{Q}$, если $M \cap \Pi$ имеет свойство $\mathrm{Q}$ для любого бруса П в $X$.

ТеОрема 9.8. Пусть $X_{n} \in(\mathrm{BM})$. Тогда следующие условия эквивалентны для любого солни, $M \subset X_{n}$ :

а) $M$ (экстремалъно) монотонно линейно связно;

b) $M$ экстремально стягиваемо (в частности, $M$ является В-стягиваеMol.M);

с) $M$ экстремально солнечно (т.е. пересечение $M$ с любым брусом и, в частности, с замкнутым шаром является солнцем или пусто);

d) существует непрерывная мультипликативная (аддитивная) в-выборка на $M$ для любого $\varepsilon>0$; 
е) для любого бруса $\Pi \subset X$ существует непрерьвная мультипликативная (аддитивная) в-выборка на множество $M \cap \Pi$ для любого $\varepsilon>0$.

Tеорема 9.9. В полиэдральном пространстве $X_{n} \in(\mathrm{BM})$ множество $M$ является солнцем тогда и только тогда, когда выполнено любое из условий а)-е) теоремы 9.8 .

Среди нерешенных проблем в этой области выделим следующие.

Провлема 15. Охарактеризовать пространства, в которых каждое (ограниченное) чебышёвское множество монотонно линейно связно, т-связно.

По известной теореме Власова [222] (см. замечание 9.3) ограниченно компактное $P$ - или $B$-ацикличное подмножество банахова пространства является солнцем. Как следствие, в конечномерных (ВМ)-пространствах $P$ - или $B$-ацикличность влечет солнечность, а значит, и монотонную линейную связность. Далее, в конечномерном полиэдральном пространстве $X_{n}$ каждое солнце монотонно линейно связно тогда и только тогда, когда $X_{n} \in(\mathrm{BM})$. В связи со сказанным сформулируем следующую проблему.

ПроБЛЕма 16. Известно, что $\ell^{1}(n) \notin(\mathrm{BM}), n \geqslant 3$, и поэтому в $\ell^{1}(n)$ существует не монотонно линейно связное солнце. Однако неизвестно, будет ли такое солнце $P$-ацикличным (или хотя бы $P$-связным).

\section{5. Солнечность и монотонная линейная связность в пространст-} вах $C(Q)$. Отправной точкой при исследовании монотонной связности в теории приближений служит теорема Браесса [57], утверждающая (в наших терминах), что строгие солнца в пространстве $\ell^{\infty}(n)$ монотонно линейно связны, а также следующий результат Беренса и Хетцельта [44] (тоже формулируемый в наших терминах).

Теорема 9.10. Hепустое подмножество пространства $\ell^{\infty}(n)$ является солнием тогда и только тогда, когда оно замкнуто и монотонно линейно связно.

В следующей теореме [11] найдено бесконечномерное негладкое пространство, не являющееся неквадратным, в котором всякое солнце связно (и, более того, монотонно линейно связно).

Теорема 9.11. 1) Произвольное солнце в с монотонно линейной связно.

2) т-связное (и тем более монотонно линейно связное) аппроксимативно компактное непустое подмножество пространства с является солнцем.

3) Пространство с с содержит замкнутое монотонно связное множество, не являющееся $\delta$-солнием.

Для случая $X=C(Q)$, где $Q$ - метрический компакт, известные результаты о связности строгих солнц и чебышёвских множеств можно усилить следующим образом [15; теорема 3].

Теорема 9.12. Ограниченно компактное строгое солнце (в частности, ограниченно компактное чебышёвское множество) в пространстве $C(Q)$ монотонно линейно связно и $B$-клеточноподобно. 
Второе утверждение в теореме 9.12 частично обращает хорошо известную теорему Власова, согласно которой ограниченно компактное Р-ацикличное подмножество банахова пространства является солнцем.

9.6. Пересечение солнц и монотонно линейно связных множеств с брусами. Вопрос об аппроксимативных и геометрических свойствах пересечений солнц и строгих солнц с промежутками (брусами) в пространствах типа $C(Q)$ рассматривался в [18], [14] и [15] (см. также [25; §7.4]). По поводу аппроксимативных свойств пересечений солнц, строгих солнц и чебышёвских множеств с сегментами на нормированной плоскости см. [16].

Напомним [24], что по определению брус - это пересечение любого семейства экстремальных гиперполос вида

$$
\{x \in X \mid a \leqslant f(x) \leqslant b\}, \quad-\infty \leqslant a \leqslant b \leqslant+\infty, \quad f \in \operatorname{ext} S^{*},
$$

порождаемых в исходном пространстве экстремальными функционалами из $S^{*}$. Отметим, что все брусы в $C(Q)$ суть замкнутые промежутки [24]. Аналогичный результат верен для множеств с непустой внутренностью в любом конечномерном пространстве $X_{n}$ (см. [19]). Включение $[x, y] \subset$ П при любых $x, y \in \Pi$ $(\Pi-$ брус) очевидно в любом $X$ (т. е. брус заведомо является замкнутым промежутком).

Брусы естественно возникают в задачах отделимости экстремальными функционалами, а также в теории приближений гладкими функциями при ограничении на производную. Интересно отметить, что брусы обладают следующим характеристическим свойством: они строго экстремально отделяются (т. е. строго отделяются экстремальным функционалом из $S^{*}$ ) от любой точки, им не принадлежащей [24]; при этом для двумерных пространств, а также для пространств типа $C(Q)$ имеет место аналог теоремы отделимости: два непересекающихся бруса строго отделяются экстремальным функционалом [24]; в общем случае (например, в $L^{1}(\Omega),|\Omega| \geqslant 3$ ) это утверждение неверно.

В теоремах 9.13-9.15 (см. [19]) даются условия, обеспечивающие солнечность пересечений солнц с брусами. Естественность бруса в данной задаче показана в теореме 9.16 .

Теорема 9.13. Пусть $\varnothing \neq M \subset X_{n}$ - замкнутое т-связное множество (в частности, $M$ - солнце в произвольном двумерном $X_{2}$ или в $X_{n} \in(\mathrm{BM})$ ), и пусть $\Pi-$ брус в $X_{n}, M \cap \Pi \neq \varnothing$. Тогда

$$
M \cap \Pi \text { - монотонно линейно связное солние в } X_{n} \text {. }
$$

Tеорема 9.14. Пусть $X \in(\mathrm{MeI}) \cap\left(\mathrm{Ex}-w^{*} \mathrm{~s}\right)$ (в частности, $X$ - сепарабельное банахово пространство), $M \neq \varnothing$ - ограниченно компактное т-связное

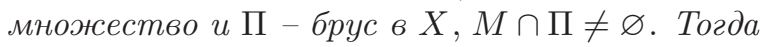

$M \cap \Pi-$ монотонно линейно связное солнце в $X$.

Tеорема 9.15. Пусть $M \neq \varnothing-$ монотонно линейно связное множество

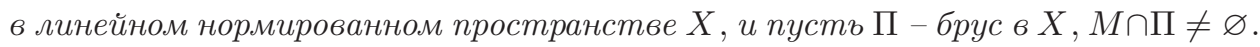
Тогда

$M \cap \Pi$ монотонно линейно связно. 
ЗАмЕчАНИЕ 9.4. Отметим существенность требования монотонной линейной связности (т-связности) солнц в теоремах 9.13-9.15. Соответственно, имея в нашем распоряжении невыпуклое чебышёвское солнце $M^{\prime}$ в $X_{n}$ из примера 9.1, мы можем найти такую прямую (брус), пересечение которой с $M^{\prime}$ несвязно и, следовательно, не может быть солнцем, поскольку по упомянутой выше теореме Кощеева-Брауна любое солнце в конечномерном пространстве связно.

В следующей теореме [19] дается характеризация строгих солнц в терминах солнечности их пересечений с замкнутыми телесными промежутками. Аналогичный результат для $C(Q)$ содержится в [18].

Теорема 9.16. Пусть $\Pi \neq \varnothing-$ замкнутое множество в $X_{n}$. Следующие утверждения эквивалентны:

а) П - брус (замкнутый промежуток);

b) $\Pi \cap M-$ монотонно линейно связное солнце для любого монотонно линейно связного солниа $M, M \cap \Pi \neq \varnothing$;

с) $\Pi \cap M-$ солнце для любого монотонно линейно связного солнца $M$ такого, что $M \cap \Pi \neq \varnothing$;

d) $\gamma \cap \Pi$ - монотонно линейно связное солнце для любой монотонной дуги $\gamma$ такой, что $\gamma \cap \Pi \neq \varnothing$.

В теореме 9.16 требование монотонной линейной связности существенно (см. замечание 9.4). Аналогичный результат для пространства $\ell^{\infty}(n)$ получен в [14] для нормированной плоскости - в [16]. Солнечность пересечений строгих солнц с замкнутыми телесными промежутками (брусами) в $C(Q)$ рассмотрена в [18].

Напомним, что любой замкнутый шар является брусом. В случае $C(Q)$, где $Q$ - метризуемый компакт, отметим следующие результаты [15], [18].

Tеорема 9.17. Пусть $M \subset C(Q)$ - ограниченно компактное строгое солнце в $C(Q)$. Предположим, что $M \cap \stackrel{\circ}{B}(\theta, r) \neq \varnothing$ для некоторых $\theta \in C(Q), r>0$. Тогда $M \cap B(\theta, r)$ - компактное строгое солнце в $C(Q)$.

ТеОРема 9.18. Имеют место следующие утверждения.

а) Пусть $M$ - строгое протосолнще в $C(Q)$, П - замкнутый телесный промежуток в $C(Q)$, причем $M \cap \operatorname{int} \Pi \neq \varnothing$. Тогда $M \cap \Pi-$ строгое протосолние в $C(Q)$.

b) Пусть $M$ - строгое протосолнце в $C(Q), \Pi$ - замкнутый телесный промежуток в $C(Q)$, причем $M \cap \operatorname{int} \Pi \neq \varnothing$ и $M \cap \Pi$ является множеством существования. Тогда $M \cap \Pi$ - строгое солнце в $C(Q)$.

с) Пусть $M$ - ограниченно компактное строгое солнце в $C(Q), \Pi$ - замкнутый промежуток в $C(Q), M \cap \Pi \neq \varnothing$. Тогда $M \cap \Pi-$ солнце в $C(Q)$.

В следующей теореме [18] дается характеризация строгих солнц в $C(Q)$ в терминах солнечности их пересечений с замкнутыми телесными промежутками.

Теорема 9.19. Следующие утверждения эквивалентны:

а) $M$ - строгое протосолние в $C(Q)$;

b) $M \cap \Pi$ - строгое протосолнце в $C(Q)$ для любого замкнутого телесного промежутка $\Pi$ в $C(Q)$ такого, что $M \cap \operatorname{int} \Pi \neq \varnothing$;

c) $M \cap \mathrm{m}(x, y)$ - строгое протосолние в $C(Q)$ для любых $x, y \in C(Q)$ таких, что $M \cap \operatorname{int} \mathrm{m}(x, y) \neq \varnothing$. 
ЗАмечАниЕ 9.5. Отметим ряд следствий из приведенных результатов. Рютин [187] при исследовании вопроса равномерной непрерывности оператора почти наилучшего обобщенного рационального приближения построил ряд примеров конечномерных подпространств $V, W$, для которых пересечение $R_{V, W} \cap B$, где

$$
R_{V, W}=\operatorname{cl}\{v / w \mid v \in V, w \in W, w>0 \text { на } Q\},
$$

компактно в $C(Q)(Q$ - связный метрический компакт). С учетом сказанного выше отсюда следует, что $R_{V, W}$ является ограниченно компактным $B$-стягиваемым солнцем. Отметим, что Рютин установил, что на это множество существует равномерно непрерывная мультипликативная $\varepsilon$-выборка $\varphi: B \rightarrow R_{V, W}$ при любых $\varepsilon>0$.

Авторы благодарны В.И. Бердышеву, С.А. Богатому, А.А. Васильевой, В. Б. Демидовичу, У. Х. Каримову, А. С. Кочурову, С. В. Конягину, Г. Г. Магарил-Ильяеву, Ю. В. Малыхину, А. В Маринову, В. Ю. Протасову, К. С. Рютину, В. М. Тихомирову, Н. И. Черных, Е. В. Щепину за полезные обсуждения.

\section{Список литературы}

[1] А.А. Аграчев, Ж.П.А. Готье, "Субримановы метрики и изопериметрические задачи в контактном случае", Труды международной конференции, посвященной 90-летию со дня рождения Л. С. Понтрягина (Москва, 31 августа-6 сентября 1998 г.), т. 3: Геометрическая теория управления, Итоги науки и техн. Сер. Соврем. матем. и ее прил. Темат. обз., 64, ВИНИТИ, М., 1999, 5-48; англ. пер.: A. A. Agrachev, J. P. A. Gauthier, "Sub-Riemannian metrics and isoperimetric problems in the contact case", J. Math. Sci. (N. Y.), 103:6 (2001), 639-663.

[2] A. Aizpuru, F. J. Garcia-Pacheco, "Some questions about rotundity and renormings in Banach spaces", J. Aust. Math. Soc., 79:1 (2005), 131-140.

[3] A. R. Alimov, "A number of connected components of sun's complement", Proceedings of the XIX workshop on function theory (Beloretsk, 1994), East J. Approx., 1:4 (1995), 419-429.

[4] A. R. Alimov, "Chebyshev set's complement", Proceedings of the XX workshop on function theory (Moscow, 1995), East J. Approx., 2:2 (1996), 215-232.

[5] А.Р. Алимов, "Чебышевские компакты на плоскости", Теория приближений. Гармонический анализ, Сборник статей, посвященный памяти профессора Сергея Борисовича Стечкина, Тр. МИАН, 219, Наука, М., 1997, 8-26; англ. пер.: A. R. Alimov, "Chebyshev compact sets in the plane", Proc. Steklov Inst. Math., 219 (1997), 2-19.

[6] А.Р. Алимов, "Всякое ли чебышевское множество выпукло?", Матем. просв., cep. 3, 2, МЦНМО, М., 1998, 155-172.

[7] A. R. Alimov, "The number of connected components of Chebishev sets and 'suns' complement", Proc. A. Razmadze Math. Inst., 117 (1998), 135-136.

[8] А.Р. Алимов, "О структуре дополнения к чебышёвским множествам", Функи. анализ и его прил., 35:3 (2001), 19-27; англ. пер.: A. R. Alimov, "On the structure of the complements of Chebyshev sets", Funct. Anal. Appl., 35:3 (2001), 176-182.

[9] А.Р. Алимов, "Геометрическая характеризация строгих солнц в пространстве $\ell^{\infty}(n) "$ ", Матем. заметки, 70:1 (2001), 3-11; англ. пер.: A. R. Alimov, "Geometrical characterization of strict suns in $\ell^{\infty}(n)$ ", Math. Notes, 70:1 (2001), 3-10. 
[10] A. R. Alimov, "Characterisations of Chebyshev sets in $c_{0}$ ", J. Approx. Theory, 129:2 (2004), 217-229.

[11] А. Р. Алимов, "Связность солнц в пространстве со", Изв. РАН. Сер. матем., 69:4 (2005), 3-18; англ. пер.: A. R. Alimov, "Connectedness of suns in the space $c_{0}$ ", Izv. Math., 69:4 (2005), 651-666.

[12] А.Р. Алимов, "Геометрическое строение чебышёвских множеств в $\ell^{\infty}(n)$ ", Функи. анализ и его прил., 39:1 (2005), 1-10; англ. пер.: A. R. Alimov, "The geometric structure of Chebyshev sets in $\ell^{\infty}(n)$ ", Funct. Anal. Appl., 39:1 (2005), $1-8$.

[13] А.Р. Алимов, "Выпуклость чебышёвских множеств, содержащихся в подпространстве", Матем. заметки, 78:1 (2005), 3-15; англ. пер.: A. R. Alimov, "Convexity of Chebyshev sets contained in a subspace", Math. Notes, 78:1 (2005), $3-13$.

[14] А.Р. Алимов, "Сохранение аппроксимативных свойств подмножеств чебышевских множеств и солнц в $\ell^{\infty}(n) "$, Изв. РАН. Сер. матем., 70:5 (2006), 3-12; англ. пер.: A. R. Alimov, "Preservation of approximative properties of subsets of Chebyshev sets and suns in $\ell^{\infty}(n)$ ", Izv. Math., 70:5 (2006), 857-866.

[15] А.Р. Алимов, "Монотонная линейная связность чебышёвских множеств в пространстве $C(Q)$ ", Матем. сб., 197:9 (2006), 3-18; англ. пер.: A. R. Alimov, "Monotone path-connectedness of Chebyshev sets in the space $C(Q)$ ", Sb. Math., 197:9 (2006), 1259-1272.

[16] А.Р. Алимов, "Сохранение аппроксимативных свойств чебышёвских множеств и солнц на плоскости", Вестн. Моск. ун-та. Сер. 1 Матем. Мех., 2008, № 4, 46-49; англ. пер.: А. R. Alimov, "Preservation of approximative properties of Chebyshev sets and suns in a plane", Moscow Univ. Math. Bull., 63:5 (2008), 198-201.

[17] А.Р. Алимов, "Монотонно линейно связное чебышёвское множество является солнцем", Матем. заметки, 91:2 (2012), 305-307; англ. пер.: A. R. Alimov, "A monotone path connected Chebyshev set is a sun", Math. Notes, 91:2 (2012), 290-292.

[18] А.Р. Алимов, "Ограниченная строгая солнечность строгих солнц в пространстве $C(Q)$ ", Вестн. Моск. ун-та. Сер. 1 Матем. Мех., 2012, №6, 16-19; англ. пер.: A. R. Alimov, "Bounded strict solar property of strict suns in the space $C(Q)$ ", Moscow Univ. Math. Bull., 68:1 (2013), 14-17.

[19] А.Р. Алимов, "Локальная солнечность солнц в линейных нормированных пространствах", Фундамент. и прикл. матем., 17:7 (2012), 3-14; англ. пер.: A. R. Alimov, "Local solarity of suns in normed linear spaces", J. Math. Sci. (N. Y.), 197:4 (2014), 447-454.

[20] А.Р. Алимов, "Монотонная линейная связность и солнечность связных по Менгеру множеств в банаховых пространствах", Изв. РАН. Сер. матем., 78:4 (2014), 3-18; англ. пер.: A. R. Alimov, "Monotone path-connectedness and solarity of Menger-connected sets in Banach spaces", Izv. Math., 78:4 (2014), 641-655.

[21] A. R. Alimov, "On finite-dimensional Banach spaces in which suns are connected", Eurasian Math. J. (to appear).

[22] A. R. Alimov, H. Berens, "Examples of Chebyshev sets in matrix spaces", J. Approx. Theory, 99:1 (1999), 44-53.

[23] А.Р. Алимов, М. И. Карлов, "Множества с внешним чебышевским слоем", $M a-$ тем. заметки, 69:2 (2001), 303-307; англ. пер.: А. R. Alimov, M. I. Karlov, "Sets with external Chebyshev layer", Math. Notes, 69:2 (2001), 269-273.

[24] А.Р. Алимов, В. Ю. Протасов, "Отделимость выпуклых множеств экстремальными гиперплоскостями", Фундамент. и прикл. матем., 17:4 (2012), 3-12; англ. пер.: A. R. Alimov, V. Yu. Protasov, "Separation of convex sets by extreme hyperplanes", J. Math. Sci. (N. Y.), 191:5 (2013), 599-604. 
[25] А.Р. Алимов, И.Г. Царьков, "Связность и другие геометрические свойства солнц и чебышёвских множеств", Фундамент. и прикл. матем., 19:4 (2014), $21-91$.

[26] D. Amir, F. Deutsch, "Suns, moons, and quasi-polyhedra", J. Approx. Theory, 6:2 (1972), 176-201.

[27] J. Andres, G. Gabor, L. Górniewicz, "Acyclicity of solution sets to functional inclusions", Nonlinear Anal., 49:5 (2002), 671-688.

[28] В.И. Арнольд, Особенности каустик и волновых фронтов, Фазис, М., 1996, x+334 с.; англ. пер.: V.I. Arnol'd, Singularities of caustics and wave fronts, Math. Appl. (Soviet Ser.), 62, Kluwer Academic Publishers Group, Dordrecht, 1990, xiv $+259 \mathrm{pp}$.

[29] V.S. Balaganskii, "An antiproximal set in a strictly convex space with Fréchet differentiable norm", Proceedings of the XX workshop on function theory (Moscow, 1995), East J. Approx., 2:2 (1996), 169-176.

[30] V.S. Balaganskii, "On the connectedness of the set of points of discontinuity of the metric projection", East J. Approx., 2:3 (1996), 263-279.

[31] В. С. Балаганский, "О выпуклых замкнутых ограниченных телах без наиболее удаленных точек, замыкание дополнения которых антипроксиминально", Тр. ИММ УрО РАН, 17, 2011, 98-104; англ. пер.: V. S. Balaganskii, "On convex closed bounded bodies without farthest points such that the closure of their complement is antiproximinal", Proc. Steklov Inst. Math. (Suppl.), 277:suppl. 1 (2012), 48-54.

[32] В. С. Балаганский, Л.П. Власов, "Проблема выпуклости чебышёвских множеств", УМН, 51:6(312) (1996), 125-188; англ. пер.: V. S. Balaganskii, L. P. Vlasov, "The problem of convexity of Chebyshev sets", Russian Math. Surveys, 51:6 (1996), $1127-1190$.

[33] М. В. Балашов, Г. Е. Иванов, “Слабо выпуклые и проксимально гладкие множества в банаховых пространствах", Изв. РАН. Сер. матем., 73:3 (2009), 23-66; англ. пер.: M. V. Balashov, G. E. Ivanov, "Weakly convex and proximally smooth sets in Banach spaces", Izv. Math., 73:3 (2009), 455-499.

[34] M. V. Balashov, D. Repovš, "Uniform convexity and the splitting problem for selections", J. Math. Anal. Appl., 360:1 (2009), 307-316.

[35] H. H. Bauschke, Xianfu Wang, Jane Ye, Xiaoming Yuan, "Bregman distances and Chebyshev sets", J. Approx. Theory, 159:1 (2009), 3-25.

[36] Б. Б. Беднов, П. А. Бородин, "Банаховы пространства, реализующие минимальные заполнения", Матем. сб., 205:4 (2014), 3-20; англ. пер.: В. В. Bednov, P. A. Borodin, "Banach spaces that realize minimal fillings", Sb. Math., 205:4 (2014), 459-475.

[37] В.И. Бердышев, "K вопросу о чебышёвских множествах", Докл. АН АзССР, 22:9 (1966), 3-5.

[38] В. И. Бердышев, "О модуле непрерывности оператора наилучшего приближения", Матем. заметки, 15:5 (1974), 797-808; англ. пер.: V.I. Berdyshev, "On the modulus of continuity of an operator of best approximation", Math. Notes, 15:5 (1974), 478-484.

[39] В. И. Бердышев, "Пространства с равномерно непрерывной метрической проекцией", Матем. заметки, 17:1 (1975), 3-12; англ. пер.: V. I. Berdyshev, "Spaces with a uniformly continuous metric projection", Math. Notes, 17:1 (1975), 3-8.

[40] В. И. Бердышев, "Непрерывность многозначного отображения, связанного с задачей минимизации функционала", Изв. АН СССР. Сер. матем., 44:3 (1980), 483-509; англ. пер.: V.I. Berdyshev, "Continuity of a multivalued mapping connected with the problem of minimizing a functional", Math. USSR-Izv., 16:3 (1981), 431-456. 
[41] В.И. Бердышев, "Варьирование нормы в задаче о наилучшем приближении", Матем. заметки, 29:2 (1981), 181-196; англ. пер.: V. I. Berdyshev, "Variation of the norms in the problem of best approximation", Math. Notes, 29:2 (1981), 95-103.

[42] В. И. Бердышев, Л. В. Петрак, Аппроксимация функиий, сжатие численной информации, приложения, УрО РАН, Екатеринбург, 1999, 297 с.

[43] H. Berens, L. Hetzelt, "Suns and contractive retracts in the plane", Теория приближений функиий (Киев, 31 мая-5 июня, 1983), Наука, М., 1987, 483-487.

[44] H. Berens, L. Hetzelt, "Die metrische Struktur der Sonnen in $\ell_{\infty}(n)$ ", Aequationes Math., 27:3 (1984), 274-287.

[45] H. Berens, L. Hetzelt, "On accretive operators on $\ell_{n}^{\infty}$ ", Pacific J. Math., 125:2 (1986), 301-315.

[46] F. Bernard, L. Thibault, "Prox-regularity of functions and sets in Banach spaces", Set-Valued Anal., 12:1-2 (2004), 25-47.

[47] J. Blatter, P. D. Morris, D. E. Wulbert, "Continuity of the set-valued metric projection", Math. Ann., 178:1 (1968), 12-24.

[48] М. Борн, Э. Вольф, Основы оптики, Наука, М., 1970, 856 с.; пер. с англ.: Principles of optics: electromagnetic theory of propagation, interference and diffraction of light, eds. M. Born, E. Wolf, Pergamon Press, London-New York-Paris-Los Angeles, 1959, xxvi+803 pp.

[49] П. А. Бородин, "Пример ограниченного аппроксимативно компактного множества, не являющегося компактным", УМН, 49:4(298) (1994), 157-158; англ. пер.: P. A. Borodin, "An example of a bounded approximately compact set that is not compact", Russian Math. Surveys, 49:4 (1994), 153-154.

[50] П. А. Бородин, "Выпуклость 2-чебышевских множеств в гильбертовом пространстве", Вестн. Моск. ун-та. Сер. 1 Матем. Мех., 2008, № 3, 16-19; англ. пер.: P.A. Borodin, "Convexity of 2-Chebyshev sets in Hilbert space", Moscow Univ. Math. Bull., 63:3 (2008), 96-98.

[51] П. А. Бородин, "Приближение наипростейшими дробями на полуоси", Матем. сб., 200:8 (2009), 25-44; англ. пер.: P. A. Borodin, "Approximation by simple partial fractions on the semi-axis", Sb. Math., 200:8 (2009), 1127-1148.

[52] П. А. Бородин, "О выпуклости $N$-чебышевских множеств", Изв. РАН. Сер. матем., 75:5 (2011), 19-46; англ. пер.: P. A. Borodin, "On the convexity of $N$-Chebyshev sets", Izv. Math., 75:5 (2011), 889-914.

[53] П.А. Бородин, "Приближение наипростейшими дробями с ограничением на полюсы", Матем. сб., 203:11 (2012), 23-40; англ. пер.: P. А. Borodin, "Approximation by simple partial fractions with constraints on the poles", Sb. Math., 203:11 (2012), 1553-1570.

[54] П.А. Бородин, "Примеры множеств с заданными аппроксимативными свойствами в WCG-пространстве", Матем. заметки, 94:5 (2013), 643-647; англ. пер.: P.A. Borodin, "Examples of sets with given approximation properties in WCG-space", Math. Notes, 94:5 (2013), 605-608.

[55] J. M. Borwein, J. D. Vanderwerff, Convex functions: constructions, characterizations and counterexamples, Encyclopedia Math. Appl., 109, Cambridge Univ. Press, Cambridge, 2010, $\mathrm{x}+521 \mathrm{pp}$.

[56] D. Braess, Nonlinear approximation theory, Springer Ser. Comput. Math., 7, Springer-Verlag, Berlin, 1986, xiv+290 pp.

[57] D. Braess, "Geometrical characterizations for nonlinear uniform approximation", J. Approx. Theory, 11:3 (1974), 260-274.

[58] W.W. Breckner, "Zur Charakterisierung von Minimallösungen", Mathematica (Cluj), 12(35) (1970), 25-38.

[59] W. W. Breckner, B. Brosowski, "Ein Kriterium zur Charakterisierung von Sonnen", Mathematica (Cluj), 13(36) (1971), 181-188. 
[60] B. E. Breckner, A. Horváth, C. Varga, "A multiplicity result for a special class of gradient-type systems with non-differentiable term", Nonlinear Anal., 70:2 (2009), 606-620.

[61] A. Brøndsted, "Convex sets and Chebyshev sets", Math. Scand., 17 (1965), 5-16.

[62] A. Brøndsted, "Convex sets and Chebyshev sets. II", Math. Scand., 18 (1966), 5-15.

[63] B. Brosowski, Nicht-lineare Tschebyscheff-Approximation, B. I. Hochschulskripten, 808/808a, Bibliographisches Institut, Mannheim, 1968, iii+153 pp.

[64] B. Brosowski, "Nichtlineare Approximation in normierten Vektorräumen", Abstract spaces and approximation (Oberwolfach, 1968), Internat. Schriftenreihe Numer. Math., 10, Birkhäuser, Basel, 1969, 140-159.

[65] B. Brosowski, "Einige Bemerkungen zum verallgemeinerten Kolmogoroffschen Kriterium", Funktionalanalytische Methoden der numerischen Mathematik (Oberwolfach, 1967), Internat. Schriftenreihe Numer. Math., 12, Birkhäuser, Basel, 1969, $25-34$.

[66] B. Brosowski, F. Deutsch, "Some new continuity concepts for metric projections", Bull. Amer. Math. Soc., 78:6 (1972), 974-978.

[67] B. Brosowski, F. Deutsch, J. Lambert, P. D. Morris, "Chebyshev sets which are not suns", Math. Ann., 212:2 (1974), 89-101.

[68] B. Brosowski, F. Deutsch, "Radial continuity of set-valued metric projection", $J$. Approx. Theory, 11:3 (1974), 236-253.

[69] B. Brosowski, F. Deutsch, "On some geometric properties of suns", J. Approx. Theory, 10:3 (1974), 245-267.

[70] B. Brosowski, R. Wegmann, "Charakterisierung bester Approximationen in normierten Vektorräumen", J. Approx. Theory, 3:4 (1970), 369-397.

[71] A. L. Brown, "Chebyshev sets and facial systems of convex sets in finite-dimensional spaces", Proc. London Math. Soc. (3), 41:2 (1980), 297-339.

[72] A. L. Brown, "Chebyshev sets and the shapes of convex bodies", Methods of functional analysis in approximation theory (Bombay, 1985), Internat. Schriftenreihe Numer. Math., 76, Birkhäuser, Basel, 1986, 97-121.

[73] A. L. Brown, "Suns in normed linear spaces which are finite dimensional", Math. Ann., 279:1 (1987), 87-101.

[74] A. L. Brown, "On the connectedness properties of suns in finite dimensional spaces", Functional analysis and optimization (Canberra, 1988), Proc. Centre Math. Anal. Austral. Nat. Univ., 20, Austral. Nat. Univ., Canberra, 1988, 1-15.

[75] A. L. Brown, "On the problem of characterising suns in finite dimensional spaces", Proceedings of the Fourth International Conference on Functional Analysis and Approximation Theory, v. I (Potenza, 2000), Rend. Circ. Mat. Palermo (2) Suppl., 68, part I, Circ. Mat. Palermo, Palermo, 2002, 315-328.

[76] A.L. Brown, "Suns in polyhedral spaces", Seminar of mathematical analysis (Malaga/Seville, 2002/2003), Colecc. Abierta, 64, Univ. Sevilla Secr. Publ., Seville, 2003, 139-146.

[77] Дж. Брус, П. Джиблин, Кривые и особенности. Геометрическое введение в теорию особенностей, Современная математика: Вводные курсы, Мир, М., 1988, 264 с.; пер. с англ.: J.W. Bruce, P. J. Giblin, Curves and singularities. A geometrical introduction to singularity theory, Cambridge Univ. Press, Cambridge, 1984, xii+222 pp.

[78] L. N.H. Bunt, Bijdrage tot de theorie der convexe puntverzamelingen, Proefschrifft Groningen, Noord-Hollandsche Uitgevers Maatschappij, Amsterdam, 1934, 108 pp.

[79] P. Butzer, F. Jongmans, "P. L. Chebyshev (1821-1894). A guide to his life and work", J. Approx. Theory, 96:1 (1999), 111-138. 
[80] S. Carl, S. Heikkilä, Fixed point theory in ordered sets and applications. From differential and integral equations to game theory, Springer, New York, 2011, xiv+477 pp.

[81] E. W. Cheney, Introduction to approximation theory, McGraw-Hill Book Co., New York-Toronto-London, 1966, xii+259 pp.

[82] К.В. Чеснокова, "Коэффициент линейности метрической проекции для одномерных чебышевских подпространств в пространстве $C$ ", Матем. заметки, 96:4 (2014), 588-595; англ. пер.: K. V. Chesnokova, "The linearity coefficient of metric projections onto one-dimensional Chebyshev subspaces of the space C", Math. Notes, 96:4 (2014), 556-562.

[83] L. Chong, G. A. Watson, "Characterization of a best and a unique best approximation from constrained rationals", Comput. Math. Appl., 30:3-6 (1995), 51-57.

[84] S. Cobzaş, "Geometric properties of Banach spaces and the existence of nearest and farthest points", Abstr. Appl. Anal., 2005:3 (2005), 259-285.

[85] Ş. Cobzaş, Functional analysis in asymmetric normed spaces, Front. Math., Birkhäuser/Springer Basel AG, Basel, 2013, x+219 pp.

[86] E. N. Dancer, B. Sims, "Weak star separability", Bull. Austral. Math. Soc., 20:2 (1979), 253-257.

[87] F. Deutsch, Best approximation in inner product spaces, CMS Books Math./Ouvrages Math. SMC, 7, Springer-Verlag, New York, 2001, xvi+338 pp.

[88] F. Deutsch, J. M. Lambert, "On continuity of metric projections", J. Approx. Theory, 29:2 (1980), 116-131.

[89] C. Dierieck, "Characterization for best nonlinear approximations: a geometrical interpretation", J. Approx. Theory, 14:3 (1975), 163-187.

[90] R. Dragoni, J. W. Macki, P. Nistri, P. Zecca, Solution sets of differential equations in abstract spaces, Pitman Research Notes in Mathematics Series, 342, Harlow, Longman, 1996, xii+100 pp.

[91] Ch. B. Dunham, "Rational Chebyshev approximation on subsets", J. Approx. Theory, 1:4 (1968), 484-487.

[92] Ch. B. Dunham, "Characterizability and uniqueness in real Chebyshev approximation", J. Approx. Theory, 2:4 (1969), 374-383.

[93] Ch. B. Dunham, "Chebyshev sets in $C[0,1]$ which are not suns", Canad. Math. Bull., 18:1 (1975), 35-37.

[94] Ch. B. Dunham, "Transformed rational Chebyshev approximation", J. Approx. Theory, 19:3 (1977), 200-204.

[95] K. Eda, U.H. Karimov, D. Repovš, "On (co)homology locally connected spaces", Topology Appl., 120:3 (2002), 397-401.

[96] Н.В. Ефимов, С.Б. Стечкин, "Некоторые свойства чебышёвских множеств", Докл. АН СССР, 118:1 (1958), 17-19.

[97] Н. В. Ефимов, С. Б. Стечкин, "Аппроксимативная компактность и чебышёвские множества", Докл. АН СССР, 140:3 (1961), 522-524; англ. пер.: N. V. Efimov, S. B. Stechkin, "Approximate compactness and Chebyshev sets", Soviet Math. Dokl., 2 (1961), 1226-1228.

[98] F. Faraci, A. Iannizzotto, "An extension of a multiplicity theorem by Ricceri with an application to a class of quasilinear equations", Studia Math., 172:3 (2006), 275-287.

[99] F. Faraci, A. Iannizzotto, "Well posed optimization problems and nonconvex Chebyshev sets in Hilbert spaces", SIAM J. Optim., 19:1 (2008), 211-216.

[100] H. Federer, "Curvature measures", Trans. Amer. Math. Soc., 93:3 (1959), 418-491.

[101] Р. Фейнман, Р. Лейтон, М. Сэндс, Фейнмановские лекции по физике, т. 3: Излучение, волны, кванты, 3-е изд., Мир, М., 1976, 240 с.; пер. с англ.: R. Р. Feynman, R. B. Leighton, M. Sands, The Feynman lectures on physics, v. I: Mainly mechanics, radiation, and heat, Addison-Wesley Publishing Co., Inc., 1963, 52 chapt.. 
[102] J. Fletcher, The Chebyshev set problem, Master of Science thesis, The Univ. of Auckland, 2013, iv+83 pp.

[103] J. Fletcher, W.B. Moors, "Chebyshev sets", J. Aust. Math. Soc., 98:2 (2015), 161-231.

[104] C. Franchetti, E. W. Cheney, "The embedding of proximinal sets", J. Approx. Theory, 48:2 (1986), 213-225.

[105] C. Franchetti, S. Roversi, Suns, M-connected sets and P-acyclic sets in Banach spaces, Preprint № 50139, Inst. di Mat. Appl. "G. Sansone", Firenze, 1988, 29 pp.

[106] А. В. Фурсиков, "Свойства решений некоторых экстремальных задач, связанных с системой Навье-Стокса”, Матем. сб., 118(160):3(7) (1982), 323-349; англ. пер.: A. V. Fursikov, "Properties of solutions of some extremal problems connected with the Navier-Stokes system", Math. USSR-Sb., 46:3 (1983), 323-351.

[107] А.В. Фурсиков, "Некоторые вопросы теории оптимального управления нелинейными системами с распределенными параметрами", Тр. сем. им. И.Г. Петровского, 1983, № 9, 167-189.

[108] А.В. Фурсиков, Оптимальное управление распределенными системами. Теория и приложения, Науч. кн., Новосибирск, 1999, хіi+352 с.; англ. пер.: A. V. Fursikov, Optimal control of distributed systems. Theory and applications, Transl. Math. Monogr., 187, Amer. Math. Soc., Providence, RI, 2000, xiv+305 pp.

[109] А. Л. Гаркави, "О существовании наилучшего приближения функции двух переменных суммами плоских волн", Теория приближений. Гармонический анализ, Сборник статей, посвященный памяти профессора Сергея Борисовича Стечкина, Тр. МИАН, 219, Наука, М., 1997, 130-136; англ. пер.: A. L. Garkavi, "On existence of best approximation of a function of two variables by sums of plane waves", Proc. Steklov Inst. Math., 219 (1997), 123-129.

[110] J.R. Giles, "The Mazur intersection problem", J. Convex Anal., 13:3-4 (2006), 739-750.

[111] В. Л. Гончаров, “Теория наилучшего приближения функций”, Научное наследие П. Л. Чебышева, Изд-во АН СССР, М.-Л., 1945, 122-172.

[112] V. L. Goncharov, "The theory of best approximation of functions", J. Approx. Theory, 106 (2000), 2-57.

[113] L. Górniewicz, "Topological structure of solution sets: current results", CDDE 2000 Proceedings (Brno), Arch. Math. (Brno), 36, suppl. (2000), 343-382.

[114] L. Górniewicz, Topological fixed point theory of multivalued mappings, 2nd ed., Topol. Fixed Point Theory Appl., 4, Springer, Dordrecht, 2006, xiv+539 pp.

[115] A. S. Granero, M. Jiménez-Sevilla, J. P. Moreno, "Intersections of closed balls and geometry of Banach spaces", Extracta Math., 19:1 (2004), 55-92.

[116] A. S. Granero, J. P. Moreno, R. R. Phelps, "Mazur sets in normed spaces", Discrete Comput Geom., 31:3 (2004), 411-420.

[117] P. M. Gruber, "Planar Chebyshev sets", Mathematical structure-computational mathematics-mathematical modelling, v.2, Publ. House Bulgar. Acad. Sci., Sofia, 1984, 184-191.

[118] А.А. Гусак, "Предыстория и начало развития теории приближения функций”, Истор.-матем. исслед., 14, ГИФМЛ, М., 1961, 289-348.

[119] А.А. Гусак, Теория приближсения функиий, БГУ, Минск, 1972, 208 с.

[120] K.P. Hart, J. Nagata, J.E. Vaughan (eds.), Encyclopedia of general topology, Elsevier, Amsterdam, 2004, x+526 pp.

[121] J.-P. Hiriart-Urruty, "Potpourri of conjectures and open questions in nonlinear analysis and optimization", SIAM Rev., 49:2 (2007), 255-273.

[122] S. T. Hu, Theory of retracts, Wayne State Univ. Press, Detroit, 1965, 234 pp. 
[123] S. Hu, N.S. Papageorgiou, Handbook of multivalued analysis, v. II: Applications, Math. Appl. (N. Y.), 500, Kluwer Acad. Publ., Dordrecht, 2000, xii+926 pp.

[124] G. E. Ivanov, "On well posed best approximation problems for a nonsymmetric seminorm", J. Convex Anal., 20:2 (2013), 501-529.

[125] Г.Е. Иванов, М. С. Лопушански, “Аппроксимативные свойства слабо выпуклых множеств в пространствах с несимметричной полунормой”, Тр. МФТИ, 4:4 (2012), 94-104.

[126] В. К. Иванов, В. В. Васин, В.П. Танана, Теория линейных некорректных задач и ее приложения, Наука, М., 1978, 206 с.; англ. пер.: V. K. Ivanov, V. V. Vasin, V. P. Tanana, Theory of linear ill-posed problems and its applications, 2nd ed., Inverse Ill-posed Probl. Ser., 36, VSP, Utrecht, 2002, xiii+281 pp.

[127] M. Jiang, "On Johnson's example of a nonconvex Chebyshev set", J. Approx. Theory, 74:2 (1993), 152-158.

[128] M. Jiménez Sevilla, J. P. Moreno, "A note on norm attaining functionals", Proc. Amer. Math. Soc., 126:7 (1998), 1989-1997.

[129] G. G. Johnson, "Closure in a Hilbert space of a prehilbert space Chebyshev set", Topology Appl., 153:2-3 (2005), 239-244.

[130] A. Jourani, L. Thibault, D. Zagrodny, "Differential properties of the Moreau envelope", J. Funct. Anal., 266:3 (2014), 1185-1237.

[131] М.И. Карлов, "Чебышевские множества на многообразиях", Тр. ИМм УрО PAH, 4, 1996, 157-161.

[132] М. И. Карлов, И. Г. Царьков, "Выпуклость и связность чебышевских множеств и солнц", Фундамент. и прикл. матем., 3:4 (1997), 967-978.

[133] С. Я. Хавинсон, “Аппроксимативные свойства некоторых множеств в пространствах непрерывных функций”, Anal. Math., 29:2 (2003), 87-105.

[134] V.L. Klee, Jr., "A characterization of convex sets", Amer. Math. Monthly, 56:4 (1949), 247-249.

[135] V. Klee, "Dispersed Chebyshev sets and coverings by balls", Math. Ann., 257:2 (1981), 251-260.

[136] V. Klee, "Do infinite-dimensional Banach spaces admit nice tilings?", Studia Sci. Math. Hungar., 21:3-4 (1986), 415-427.

[137] H. König, "A general minimax theorem based on connectedness", Arch. Math. (Basel), 59:1 (1992), 55-64.

[138] С. В. Конягин, "Связность множеств в задачах наилучшего приближения", Докл. АН СССР, 261:1 (1981), 20-23; англ. пер.: S. V. Konyagin, "Connectedness of sets in best approximation problems", Soviet Math. Dokl., 24 (1981), 460-463.

[139] С. В. Конягин, "О множествах точек непустоты и непрерывности метрической проекции", Матем. заметки, 33:5 (1983), 641-655; англ. пер.: S. V. Konyagin, "Sets of points of nonemptiness and continuity of the metric projection", Math. Notes, 33:5 (1983), 331-338.

[140] С. В. Конягин, "О непрерывности оператора обобщенного рационального приближения", Матем. заметки, 44:3 (1988), 404.

[141] С. В. Конягин, "Об аппроксимативных свойствах произвольных замкнутых множеств в банаховых пространствах", Фундамент. и прикл. матем., 3:4 (1997), 979-989.

[142] С. В. Конягин, И.Г. Царьков, "Пространства Ефимова-Стечкина", Вестн. Моск. ун-та. Сер. 1 Матем. Мех., 1986, № 5, 20-27; англ. пер.: S. V. Konyagin, I. G. Tsar'kov, Moscow Univ. Math. Bull., 41:5 (1986), 20-28.

[143] В. А. Кощеев, "Связность и аппроксимативные свойства множеств в линейных нормированных пространствах", Матем. заметки, 17:2 (1975), 193-204; англ. пер.: V.A. Koshcheev, "The connectivity and approximative properties of sets in linear normed spaces", Math. Notes, 17:2 (1975), 114-119. 
[144] Б. А. Кощеев, "Связность и солнечные свойства множеств в линейных нормированных пространствах", Матем. заметки, 19:2 (1976), 267-278; англ. пер.: B. A. Koščeev, "Connectedness and solar properties of sets in normed linear spaces", Math. Notes, 19:2 (1976), 158-164.

[145] В. А. Кощеев, "Некоторые свойства $\delta$-проекции в линейных нормированных пространствах", Изв. вузов. Матем., 1976, № 5, 36-42; англ. пер.: V. A. Koshcheev, "Some properties of the $\delta$-projection in linear normed spaces", Soviet Math. (Iz. VUZ), 20:5 (1976), 26-30.

[146] В.А. Кощеев, "Пример несвязного солнца в банаховом пространстве", Maтем. заметки, 26:1 (1979), 89-92; англ. пер.: V.A. Koshcheev, "An example of a disconnected sun in a Banach space", Math. Notes, 26:1 (1979), 535-537.

[147] V.A. Koshcheev, "On the structure of suns in Banach spaces", Approximation and function spaces (Gdańsk, 1979), North-Holland, Amsterdam-New York, 1981, 371-376.

[148] В. А. Кощеев, "Связность по Власову-Вулберту множеств в гильбертовом пространстве", Матем. заметки, 80:5 (2006), 790-792; англ. пер.: V. A. Koshcheev, "Vlasov-Wulbert connectedness of sets in Hilbert space", Math. Notes, 80:5 (2006), $744-747$.

[149] Ю. А. Кравцов, Ю. И. Орлов, Геометрическая оптика неоднородных сред, Наука, М., 1980, 304 с.

[150] С. Н. Кружков, “Обобщенные решения уравнений Гамильтона-Якоби типа эйконала. I. Постановка задач, теоремы существования, единственности и устойчивости, некоторые свойства решений”, Матем. сб., 98(140):3(11) (1975), 450-493; англ. пер.: S. N. Kruzhkov, "Generalized solutions of the Hamilton-Jacobi equations of eikonal type. I. Formulation of the problems; existence, uniqueness and stability theorems; some properties of the solutions", Math. USSR-Sb., 27:3 (1975), 406-446.

[151] W. Kryszewski, "On the existence of equilibria and fixed points of maps under constraints", Handbook of topological fixed point theory, Springer, Dordrecht, 2005, 783-866.

[152] П. Д. Лебедев, А.А. Успенский, В.Н. Ушаков, "Построение минимаксного решения уравнения типа эйконала", Тр. ИММ УрО РАН, 14, 2008, 182-191; англ. пер.: P.D. Lebedev, A. A. Uspenskii, V.N. Ushakov, "Construction of a minimax solution for an eikonal-type equation", Proc. Steklov Inst. Math. (Suppl.), 263:suppl. 2 (2008), S191-S201.

[153] Е. Д. Лившиц, "Об устойчивости оператора $\varepsilon$-проекции на множество сплайнов в пространстве C[0,1]", Изв. РАН. Сер. матем., 67:1 (2003), 99-130; англ. пер.: E.D. Livshits, "Stability of the operator of $\varepsilon$-projection to the set of splines in $C[0,1] "$, Izv. Math., 67:1 (2003), 91-119.

[154] Е. Д. Лившиц, "О почти наилучшем приближении кусочно-полиномиальными функциями в пространстве $C[0,1]$ ", Матем. заметки, 78:4 (2005), 629-633; англ. пер.: E. D. Livshits, "On almost-best approximation by piecewise polynomial functions in the space $C[0,1]$ ", Math. Notes, 78:4 (2005), 586-591.

[155] E. D. Livshits, "Continuous selections of operators of almost best approximation by splines in the space $L_{p}[0,1]$. I", Russ. J. Math. Phys., 12:2 (2005), 215-218.

[156] H. Maehly, Ch. Witzgall, "Tschebyscheff-Approximationen in kleinen Intervallen. II. Stetigkeitssätze für gebrochen rationale Approximationen", Numer. Math., 2 (1960), 293-307.

[157] Ю.В.Малыхин, "Условие выпуклости в теоремах Кукера-Смейла в теории обучения", Матем. заметки, 84:1 (2008), 144-148; англ. пер.: Yu. V. Malykhin, "Convexity condition in Cucker-Smale theorems in the theory of teaching", Math. Notes, 84:1 (2008), 142-146. 
[158] H. Mann, "Untersuchungen über Wabenzellen bei allgemeiner Minkowskischer Metrik", Monatsh. Math. Phys., 42 (1935), 417-424.

[159] А. В. Маринов, "Непрерывность и связность метрической $\delta$-проекции", Аппроксимация в конкретных и абстрактных банаховых пространствах, Сборник статей, УНЦ АН СССР, Свердловск, 1987, 82-95.

[160] А.В. Маринов, "Оценки устойчивости непрерывной селекции для метрической почти-проекции", Матем. заметки, 55:4 (1994), 47-53; англ. пер.: A. V. Marinov, "Stability estimates of continuous selections for metric almostprojections", Math. Notes, 55:4 (1994), 367-371.

[161] А.В. Маринов, "Константы Липшица оператора метрического $\varepsilon$-проектирования в пространствах с заданными модулями выпуклости и гладкости", Изв. РАН. Сер. матем., 62:2 (1998), 103-130; англ. пер.: А. V. Marinov, "The Lipschitz constants of the metric $\varepsilon$-projection operator in spaces with given modules of convexity and smoothness", Izv. Math., 62:2 (1998), 313-318.

[162] У. Масси, Теория гомологий и когомологий. Подход, основанный на применении коцепей Александера-Спенъера, Мир, M., 1981, 389 с.; пер. с англ.: W. S. Massey, Homology and cohomology theory. An approach based on Alexander-Spanier cochains, Monogr. Textbooks Pure Appl. Math., 46, Marcel Dekker, Inc., New York-Basel, 1978, xiv+412 pp.

[163] R. E. Megginson, An introduction to Banach space theory, Grad. Texts in Math., 183, Springer-Verlag, New York, 1998, xx+596 pp.

[164] G. Meinardus, D. Schwedt, "Nicht-lineare Approximationen", Arch. Ration. Mech. Anal., 17:4 (1964), 297-326.

[165] С.А. Мелихов, "Стинродовские гомотопии”, УМH, 64:3(387) (2009), 73-166; англ. пер.: S. A. Melikhov, "Steenrod homotopy", Russian Math. Surveys, 64:3 (2009), 469-551.

[166] K. Menger, "Untersuchungen über allgemeine Metrik", Math. Ann., 100:1 (1928), $75-163$.

[167] J.P. Moreno, R. Schneider, "Continuity properties of the ball hull mapping", Nonlinear Anal., 66:4 (2007), 914-925.

[168] T. Motzkin, "Sur quelques propriétés caractéristiques des ensembles convexes", Atti Accad. Naz. Lincei Rend. (6), 21 (1935), 562-567.

[169] Н.В. Невесенко, "Строгие солнца и полунепрерывность метрической проекции в линейных нормированных пространствах", Матем. заметки, 23:4 (1978), 563-572; англ. пер.: N. V. Nevesenko, "Strict sums and semicontinuity below metric projections in linear normed spaces", Math. Notes, 23:4 (1978), 308-312.

[170] Н. В. Невесенко, "Метрическая проекция и связность множеств в линейных нормированных пространствах", Изв. вузов. Матем., 1979, № 1, 51-53; англ. пер.: N.V. Nevesenko, "Metric projection and connectedness of sets in linear normed spaces", Soviet Math. (Iz. VUZ), 23:1 (1979), 40-42.

[171] G. Nürnberger, "Strongly unique spline approximation with free knots", Constr. Approx., 3:1 (1987), 31-42.

[172] G. Nürnberger, Approximation by spline functions, Springer-Verlag, Berlin, 1989, xii+243 pp.

[173] Е.В. Ошман, “Чебышевские множества и непрерывность метрической проекции", Изв. вузов. Матем., 1970, № 9, 78-82.

[174] S. Papadopoulou, "A connected separable metric space with a dispersed Chebyshev set”, J. Approx. Theory, 39:4 (1983), 320-323.

[175] Chr. Pauc, "Sur la rélation entre un point et une de ses projections sur un ensemble", Revue Sci. (Rev. Rose Illus.), 77 (1939), 657-658.

[176] R. R. Phelps, "A representation theorem for bounded convex sets", Proc. Amer. Math. Soc., 11:6 (1960), 976-983. 
[177] R. A. Poliquin, R. T. Rockafellar, "Prox-regular functions in variational analysis", Trans. Amer. Math. Soc., 348:5 (1996), 1805-1838.

[178] W. Pollul, Topologien auf Mengen von Teilmengen und Stetigkeit von mengenwertigen metrischen Projektionen, Diplomarbeit, Bonn, 1967.

[179] Е.С. Половинкин, М.В. Балашов, Элементы выпуклого и сильно выпуклого анализа, Физматлит, М., 2004, 416 с.

[180] Т. Постон, И. Стюарт, Теория катастроф и ее приложения, Мир, М., 1980, 608 с.; пер. с англ.: Т. Poston, I. Stewart, Catastrophe theory and its applications, Surveys and Reference Works in Mathematics, 2, Pitman, London-San Francisco, CA-Melbourne, 1978, xviii+491 pp.

[181] D. Repovš, P. V. Semenov, "Continuous selections of multivalued mappings", Recent progress in general topology. III, Atlantis Press, Paris, 2014, 711-749.

[182] B. Ricceri, "A general multiplicity theorem for certain nonlinear equations in Hilbert spaces", Proc. Amer. Math. Soc., 133:11 (2005), 3255-3261.

[183] B. Ricceri, "Recent advances in minimax theory and applications", Pareto optimality, game theory and equilibria, Springer Optim. Appl., 17, Springer, New York, 2008, 23-52.

[184] B. Ricceri, "A conjecture implying the existence of non-convex Chebyshev sets in infinite-dimensional Hilbert spaces", Matematiche (Catania), 65:2 (2010), 193-199.

[185] К. С. Рютин, “Липшицевость ретракций и оператор обобщенного рационального приближения", Фундамент. и прикл. матем., 6:4 (2000), 1205-1220.

[186] К. С. Рютин, Аппроксимативные свойства обобщенных рациональных функций, Дисс. ... канд. физ.-матем. наук, МГУ, М., 2002, 92 с.

[187] К. С. Рютин, "О равномерно непрерывных операторах почти наилучшего обобщенного рационального приближения", Матем. заметки, 87:1 (2010), 147-150; англ. пер.: C. S. Ryutin, "On uniformly continuous almost best generalized rational approximation operators", Math. Notes, 87:1 (2010), 141-145.

[188] E. Schmidt, "Stetigkeitsaussagen bei der Tschebyscheff-Approximation mit Exponentialsummen", Math. Z., 113:2 (1970), 159-170.

[189] P. Schwartz, "Two theorems on suns in continuous function spaces", Approximation theory (Univ. Texas, Austin, TX, 1973), Academic Press, New York, 1973, 477-480.

[190] P. Shvartsman, "Lipschitz selections of set-valued mappings and Helly's theorem", J. Geom. Anal., 12:2 (2002), 289-324.

[191] L. L. Schumaker, Spline functions: basic theory, 3rd ed., Cambridge Math. Lib., Cambridge Univ. Press, Cambridge, 2007, xvi+582 pp.

[192] I. Singer, "Some remarks on approximative compactness", Rev. Roumaine Math. Pures Appl., 9 (1964), 167-177.

[193] I. Singer, Best approximation in normed linear spaces by elements of linear subspaces, Grundlehren Math. Wiss., 171, Publishing House of the Academy of the Socialist Republic of Romania, Bucharest; Springer-Verlag, New York-Berlin, 1970, 415 pp.

[194] I. Singer, The theory of best approximation and functional analysis, CBMS-NSF Regional Conf. Ser. in Appl. Math., 13, SIAM Society for Industrial and Applied Mathematics, Philadelphia, PA, 1974, vii+95 pp.

[195] В.Н. Соловьев, "О субдифференциале и производных по направлениям максимума семейства выпуклых функций”, Изв. РАН. Сер. матем., 62:4 (1998), 173-200; англ. пер.: V.N. Solov'ev, "The subdifferential and the directional derivatives of the maximum of a family of convex functions", Izv. Math., 62:4 (1998), 807-832.

[196] M. Sommer, "Charakterisierung von Minimallösungen in normierten Vektorräumen durch Eigenschaften von Hyperebenen", 14:2 (1975), 103-114. 
[197] Е.Н. Сосов, "О непрерывности и связности метрической $\delta$-проекции в равномерно выпуклом геодезическом пространстве", Изв. вузов. Матем., 2001, № 3, 55-59; англ. пер.: E. N. Sosov, "On the continuity and connectedness of metric $\delta$-projection in a uniformly convex geodesic space", Russian Math. (Iz. VUZ), 45:3 (2001), 52-56.

[198] K.-G. Steffens, The history of approximation theory. From Euler to Bernstein, Birkhäuser Boston, Inc., Boston, MA, 2006, xx+219 pp.

[199] А.И. Субботин, "Минимаксные решения уравнений Гамильтона-Якоби", Трудъ международной конференции, посвященной 90-летию со дня рождения Л. С. Понтрягина, т. 3: Геометрическая теория управления (Москва, 31 августа - 6 сентября 1998 г.), Итоги науки и техн. Сер. Соврем. матем. и ее прил. Темат. обз., 64, ВИНИТИ, М., 1999, 222-231; англ. пер.: А. I. Subbotin, "Minimax solutions to the Hamilton-Jacobi equations", J. Math. Sci. (N. Y.), 103:6 (2001), $772-777$.

[200] В. М. Тихомиров, “Теория приближений”, Анализ - 2, Итоги науки и техн. Сер. Соврем. пробл. матем. Фундам. направления, 14, ВИНИТИ, М., 1987, 103-260; англ. пер.: V. M. Tikhomirov, "Approximation theory", Analysis II. Convex analysis and approximation theory, Encyclopaedia Math. Sci., 14, Springer-Verlag, Berlin, 1990, 93-243.

[201] С. Л. Троянски, "Пример гладкого пространства, сопряженное к которому не является строго нормированным", Studia Math., 35 (1970), 305-309.

[202] И.Г. Царьков, "Ограниченные чебышевские множества в конечномерных банаховых пространствах", Матем. заметки, 36:1 (1984), 73-87; англ. пер.: I. G. Tsar'kov, "Bounded Chebyshev sets in finite-dimensional Banach spaces", Math. Notes, 36:1 (1984), 530-537.

[203] И.Г. Царьков, "О связности некоторых классов множеств в банаховых пространствах", Матем. заметки, 40:2 (1986), 174-196; англ. пер.: I. G. Tsar'kov, "Relations between certain classes of sets in Banach spaces", Math. Notes, 40:2 (1986), 597-610.

[204] И.Г. Царьков, "О компактных и слабо компактных чебышевских множествах в линейных нормированных пространствах", Докл. АН СССР, 300:1 (1988), 41-42; англ. пер.: I. G. Tsar'kov, "On compact and weakly compact Tchebycheff sets in normed linear spaces", Soviet Math. Dokl., 37:3 (1988), 629-631.

[205] И. Г. Царьков, "Компактные и слабо компактные чебышевские множества в линейных нормированных пространствах" (Душанбе, август 1986 г.), Тр. МИАН CCCP, 189 (1989), 169-184; англ. пер.: I. G. Tsar'kov, "Compact and weakly compact Tchebysheff sets in normed linear spaces", Proc. Steklov Inst. Math., 189 (1990), 199-215.

[206] И. Г. Царьков, “Локальная 'однородность' множества единственности”, Матем. заметки, 45:5 (1989), 121-123.

[207] И. Г. Царьков, "Непрерывность метрической проекции, структурные и аппроксимативные свойства множеств", Матем. заметки, 47:2 (1990), 137-148; англ. пер.: I. G. Tsar'kov, "Continuity of the metric projection, structural and approximate properties of sets", Math. Notes, 47:2 (1990), 218-227.

[208] И. Г. Царьков, "Свойства множеств, обладающих непрерывной выборкой из оператора $P^{\delta ", ~ М а т е м . ~ з а м е т к и, ~ 48: 4 ~(1990), ~ 122-131 ; ~ а н г л . ~ п е р .: ~ I . ~ G . ~ T s a r ' k o v, ~}$ "Properties of the sets that have a continuous selection from the operator $P^{\delta}$ ", Math. Notes, 48:4 (1990), 1052-1058.

[209] И.Г. Царьков, "Неединственность решений некоторых дифференциальных уравнений и их связь с геометрической теорией приближения", Матем. заметки, 75:2 (2004), 287-301; англ. пер.: I. G. Tsar'kov, "Nonunique solvability of certain differential equations and their connection with geometric approximation theory", Math. Notes, 75:2 (2004), 259-271. 
[210] И. Г. Царьков, "Устойчивость однозначной разрешимости для некоторых дифференциальных уравнений”, Тр. ИММ УрО РАН, 14, 2008, 170-182; англ. пер.: I. G. Tsar'kov, "Stability of the unique solvability for some differential equations", Proc. Steklov Inst. Math. (Suppl.), 264:suppl. 1 (2009), S185-S198.

[211] И. Г. Царьков, “Свойства множеств, обладающих устойчивой $\varepsilon$-выборкой”, $M a-$ тем. заметки, 89:4 (2011), 608-613; англ. пер.: I. G. Tsar'kov, "Properties of sets admitting stable $\varepsilon$-selections", Math. Notes, 89:4 (2011), 572-576.

[212] И.Г. Царьков, “Аппроксимативная компактность и неединственность в вариационных задачах и их приложения к дифференциальным уравнениям", $M a$ тем. сб., 202:6 (2011), 133-158; англ. пер.: I. G. Tsar'kov, "Approximative compactness and nonuniqueness in variational problems, and applications to differential equations", Sb. Math., 202:6 (2011), 909-934.

[213] И. Г. Царьков, "Устойчивость однозначной разрешимости квазилинейных уравнений по дополнительной информации”, Матем. заметки, 90:6 (2011), 918-946; англ. пер.: I. G. Tsar'kov, "Stability of unique solvability of quasilinear equations given additional data", Math. Notes, 90:6 (2011), 894-919.

[214] И. Г. Царьков, "Непрерывная в-выборка", Матем. сб., 207:2 (2016), 123-142.

[215] А. А. Васильева, "Замкнутые промежутки в векторнозначных функциональных пространствах и их аппроксимативные свойства", Изв. РАН. Сер. матем., 68:4 (2004), 75-116; англ. пер.: A. A. Vasil'eva, "Closed spans in vector-valued function spaces and their approximative properties", Izv. Math., 68:4 (2004), 709-747.

[216] А.А. Васильева, "Критерий существования гладкой функции при ограничениях", Матем. заметки, 82:3 (2007), 335-346; англ. пер.: A. A. Vasil'eva, "An existence criterion for a smooth function under constraints", Math. Notes, 82:3 (2007), 295-308.

[217] Л.П. Власов, “Аппроксимативно выпуклые множества в банаховых пространствах", Докл. АН ССCP, 163 (1965), 18-21; англ. пер.: L.P. Vlasov, "Approximately convex sets in Banach spaces", Soviet Math. Dokl., 6 (1965), 876-879.

[218] Л.П. Власов, Чебышёвские множества и их обобщения, Дисс. ... канд. физ.матем. наук, Уральский гос. ун-т, Свердловск, 1967.

[219] Л. П. Власов, “О чебышевских и аппроксимативно выпуклых множествах", Maтем. заметки, 2:2 (1967), 191-200; англ. пер.: L. P. Vlasov, "Chebyshev sets and approximately convex sets", Math. Notes, 2:2 (1967), 600-605.

[220] Л.П. Власов, "Чебышевские множества и некоторые их обобщения", Матем. заметки, 3:1 (1968), 59-69; англ. пер.: L. P. Vlasov, "Chebyshev sets and some generalizations of them", Math. Notes, 3:1 (1968), 36-41.

[221] Л.П. Власов, "Аппроксимативные свойства множеств в банаховых пространствах", Матем. заметки, 7:5 (1970), 593-604; англ. пер.: L.P. Vlasov, "Approximate properties of sets in Banach spaces", Math. Notes, 7:5 (1970), 358-364.

[222] Л.П. Власов, “Аппроксимативные свойства множеств в линейных нормированных пространствах", УМH, 28:6(174) (1973), 3-66; англ. пер.: L. P. Vlasov, "Approximative properties of sets in normed linear shaces", Russian Math. Surveys, 28:6 (1973), 1-66.

[223] Л.П. Власов, "О почти выпуклых множествах", Матем. заметки, 18:3 (1975), 343-356; англ. пер.: L. P. Vlasov, "Almost convex sets", Math. Notes, 18:3 (1975), 791-799.

[224] L. P. Vlasov, “'Suns' and geometric properties of the unit sphere in a Banach space”, Тр. ИММ УрО РАН, 5, 1998, 247-253.

[225] R. Wegmann, "Some properties of the peak-set-mapping", J. Approx. Theory, 8:3 (1973), 262-284. 
[226] D. E. Wulbert, Continuity of metric projections. Approximation theory in a normed linear lattice, Thesis (Ph. D.), Univ. Texas, Austin, 1966, 111 pp.

[227] D. E. Wulbert, "Continuity of metric projections", Trans. Amer. Math. Soc., 134:2 (1968), 335-341.

[228] W. Yang, C. Li, G. A. Watson, "Characterization and uniqueness of nonlinear uniform approximation", Proc. Edinburgh Math. Soc. (2), 40:3 (1997), 473-482.

[229] М.В. Яшина, "О плотности множества некоторых экстремальных задач", Вестн. Моск. ун-та. Сер. 1 Матем. Мех., 1986, №6, 54-56; англ. пер.: M. V. Yashina, "The density of the uniqueness set of some extremal problems", Moskow Univ. Math. Bull., 41:6 (1986), 41-44.

[230] М. В. Яшина, О единственности решения нелинейных задач управления системами с распределенными параметрами, Дисс. ... канд. физ.-матем. наук, М., МГУ, 1989.

[231] L. Zajíček, "On $\sigma$-porous sets in abstract spaces", Abstr. Appl. Anal., 2005:5 (2005), 509-534.

\section{Алексей Ростиславович Алимов} (Alexey R. Alimov)

Московский государственный университет

им. М. В. Ломоносова

E-mail: alexey.alimov-msu@yandex.ru

\section{Игорь Германович Царьков}

\section{(Igor G. Tsar'kov)}

Московский государственный университет

им. М. В. Ломоносова

E-mail: tsar@mech.math.msu.su 